\title{
Integrated condition-based planning of production and utility systems under uncertainty
}

Nur I. Zulkafli a,b and Georgios M. Kopanos ${ }^{\mathrm{a}, *}$

${ }^{a}$ Cranfield University, School of Water, Energy and Environment, Bedfordshire MK43 0AL, United Kingdom.

${ }^{\mathrm{b}}$ Universiti Teknikal Malaysia Melaka, Centre of Advanced Research on Energy, Durian Tunggal, Melaka 76100, Malaysia.

*Corresponding author: g.kopanos@cranfield.ac.uk

\begin{abstract}
A general rolling horizon optimization framework for the integrated condition-based operational and maintenance planning of production and utility systems in process industries is presented. In brief, the proposed optimization framework considers for the production and utility units: (i) improved unit performance degradation and recovery models that depend on both the cumulative time of operation and the unit operating levels deviation of units; (ii) modified operating capacities under online cleaning periods; (iii) different types of cleaning tasks (flexible time-window and online or offline condition-based); (iv) alternative options for offline cleaning tasks; (v) limited availability of cleaning resources; (vi) the initial state of the overall system at the beginning of each planning horizon; and (vii) terminal constraints for the rolling horizon problem. Total cost constitutes the objective function of the resulting problem and includes unit operating costs, cleaning costs, energy consumption costs and resource purchases costs. The case studies solved show that when compared to solutions obtained by sequential approaches the proposed integrated approach provides significantly better solutions in terms of total costs (reduction from 5\%-32\%), and especially in cost terms related to utility units operation, energy consumption, cleaning and startup/shutdown operations. Unnecessary cleanings and purchases of resources can be avoided by the proposed integrated approach.
\end{abstract}


Overall, the significant reduction in total costs is a direct result of the enhanced energy efficiency of the overall system through the efficient generation and use of energy, the improved utilization of energy and material resources resulting in a more sustainable and cleaner production practices.

Keywords: production planning; maintenance; cleaning; utility systems; rolling horizon; optimization; combined heat and power.

\section{Introduction}

One of the main goals of any process industry is to generate maximum revenues at low costs by maintaining high production levels in order to satisfy the demand for products. A means for achieving this is by following a plant-wide approach through the integrated management of operational and maintenance tasks in the overall process system (Zulkafli and Kopanos, 2016). Major industrial facilities consist of interconnected production and utility systems. Figure 1 displays a representative layout of production and utility systems for a process industry. Under this plant layout, the production system produces desired products from raw materials that may undergo several production processes, such as reactions or separations. These main production processes require large amounts of different utilities, such as power, steam, compressed air, industrial gases or water. Especially, energy intensive process industries have an onsite utility system that generate the major utilities required by the main production system. Combined heat and power units, gas or steam turbines, compressors, and boilers are examples of onsite utility systems. The raw materials of the utility system can be any type of fuel or other resource, such as atmospheric air or water. These materials undergo a conversion process in utility units to generate the desired utilities. Depending on the type of utility, chemical or physical conversion could take place in a utility unit (e.g., combustion or compression). Then, the generated utilities are supplied to the production system for its own operation and the production of intermediate or final products. Excessive amounts of utilities can be stored in buffer tanks (e.g., hot water), 
be recycled (e.g., steam), or in some cases be released to the environment (e.g., exhaust heat). Some utilities may be acquired from external sources under an associated cost, if the onsite utility system cannot meet the needs of the production system (e.g., electricity from the power grid). Production and utility units may operate in parallel or in series depending on the overall process of their corresponding production or utility system. Final products or utilities can be stored in dedicated inventory tanks or directly satisfy the demand for products or the utility requirements of the production system, respectively.

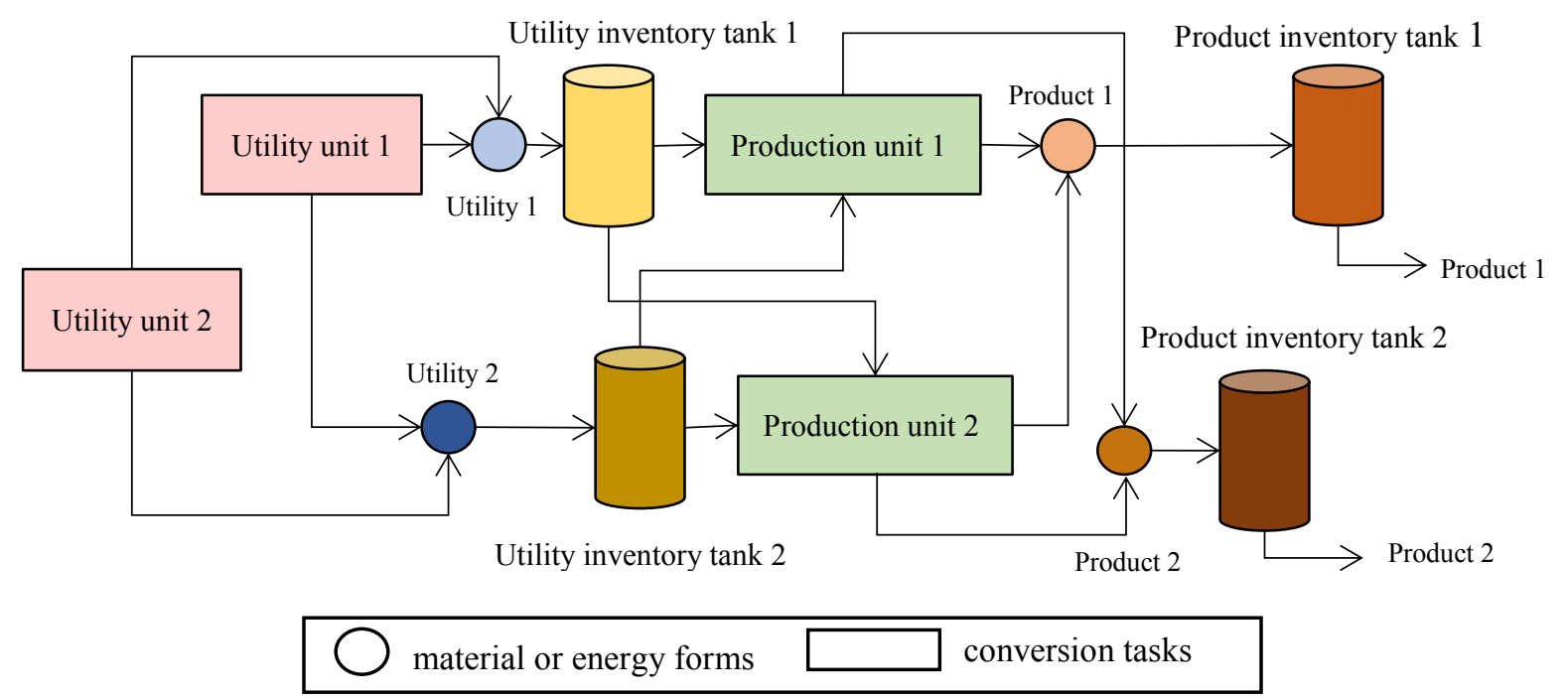

Figure 1. Representative layout for the interaction of production and utility systems.

In addition to the above, modern process plants consist of complex operating equipment that require maintenance to perform its required function in a timely manner to avoid equipment damage and inefficient use. Effective maintenance policies can sustain the operational level, reduce operating costs, and restrain the equipment and the overall system from entering hazardous states. The cleaning of production or utility equipment that are subject to performance degradation is one of the major maintenance actions in process industries. The purpose of this cleaning is to recover the performance (efficiency) of the corresponding equipment and decrease energy consumption over its operation. Thus, it is essential to consider condition-based maintenance policies for the equipment of a process plant to increase its overall energy efficiency, operability and stability (Xenos et al., 2016). To do this, performance 
degradation and recovery models need to be derived for each equipment and alternative maintenance policies need to be considered (e.g., online or offline cleaning).

Nowadays, process industries typically follow a sequential approach for the optimization of the operational plan of their production and utility systems. In this sequential approach, the planning of the production system is performed first by considering just upper bounds on the availability of utilities per time period. Once the production plan is derived, the utility needs of each production unit are known. This information is then used for obtaining the operational plan of the utility system. The main drawback of this approach is that it provides suboptimal solutions (with respect to energy efficiency and costs) since the two interconnected systems are not optimized simultaneously. Importantly, this traditional approach often faces the risk of providing generation targets for utilities that cannot be met by the utility system (infeasible solutions), and in that case either purchases of utilities would take place or a re-planning of the production may be needed (Zulkafli and Kopanos, 2016). Additionally, maintenance of production or utility units are typically predefined or follow a very conservative plan and not optimized by considering the actual operational plan of the overall process system.

In fact, most of the previous studies found in the literature have addressed individually the operational planning problem of production systems or the operational planning of utility systems. There are many works that addressed only the operational planning problem of production systems. For example, Shrouf et al. (2014) studied the production scheduling of a single machine to minimize energy consumption cost. Modarres and Izadpanahi (2016) presented a production planning model for a manufacturing plant considering energy planning, demand and production capacity. Ardjmand et al. (2016) proposed a multi-product production planning model for production plants under demand uncertainty. Zhou et al. (2017) developed production scheduling models for the textile industry. Other works focused only on the operational planning of utility systems. For example, Jin et al. (2015) developed a mixed 
integer programming model for the planning of power generation plants. Kopanos et al. (2015) presented an optimization framework for the operational and maintenance planning of compressors network in industrial air separation plants. Zhen et al. (2016) proposed a stochastic modelling approach for the planning of electric power systems. Chaturvedi et al. (2016) produced optimum water network schedule for multiple water resources. Abdul Aziz et al. (2017) studied the operational planning considering the integration of heat, cogeneration and power in industrial sites by using pinch analysis to reduce carbon emissions.

Also, some other works studied the maintenance planning (e.g., cleaning or repairing) of either production or utility systems. Some representative works on the maintenance planning for production systems are presented below. Nguyen and Bagajewicz (2010) developed preventive maintenance planning models for chemical process plants. Huang and $\mathrm{Yu},(2016)$ studied the maintenance planning problem with the objectives to reduce energy consumption and minimize makespan. Tayyab and Sarkar (2016) presented optimal batch size planning for manufacturing process to minimize total costs. Other works focused on the maintenance planning for utility systems. For instance, Cheung and Hui (2004) developed maintenance planning for industrial heat and power plant. Sanaye and Niroomand (2007) presented cleaning scheduling approaches for heat exchanger networks. Li and Nilkitsaranont (2009) studied the condition-based maintenance scheduling of gas turbine operations. Castro et al. (2014) addressed the optimal maintenance planning of a gas engine power plant.

In general, the operational or maintenance planning for utility or production systems have been studied separately in the literature. There are few works that dealt with the simultaneous operational and maintenance planning of production or utility systems. For example, Goel et al. (2003) proposed a production scheduling and maintenance optimization framework for a multi-period process plant considering process configuration, optimal production planning and different types of maintenance policies. In another study, Lavaja and Bagajewicz (2004) 
presented a mixed integer linear model for the planning of heat exchanger cleaning in chemical plants under performance degradation due to fouling. Their results showed that the production rate of the production system could increase by considering operational (e.g., performance degradation, fouling) and maintenance aspects (e.g., units cleaning and repairing) for the production units. Zhang and Hua (2007) presented a multi-period model for the simultaneous planning of the process and the utility system of a refinery complex. The application of their approach in an industrial example demonstrated an important increase in the total energy efficiency of the refinery. Finally, Agha et al. (2010) developed a mixed integer linear programming model for the simultaneous operational planning of production and utility system. Comparisons with solutions obtained by using a sequential approach indicated that the integrated approach leads to significant reduction in energy costs and at the same time decreases the emissions of harmful gases. A more detailed literature review on the operational and maintenance planning problem of production and utility systems can be found in Zulkafli and Kopanos (2016).

It is clear from the above discussion that a systematic approach is needed for addressing the plant-wide management and planning of a process industry. In addition, none of the above works on integrated planning of production and utility systems studied about condition-based and resource-focused approaches for operational and maintenance planning and follows a rolling horizon modelling representation in order to readily deal with various types of uncertainty. For this reason, this study focuses on the integrated planning of production and utility systems, where both systems are optimized simultaneously. The novelty of the proposed method follows a plant-wide condition-based approach for maintenance actions and a plantwide resource-focused approach towards the improved utilization of all process-related major resources (plant-wide resource efficiency). This integrated approach is a key step towards the transformation of current process industries to smart process industries, following the Internet- 
of-Things revolution, where all operations are performed to achieve substantially enhanced energy, sustainability, environmental and economic performance.

This study is a major extension of our previous work (Zulkafli and Kopanos, 2016) by: (i) developing an improved unit performance degradation model that considers the operating level deviations of the unit (i.e., load) along with its cumulative time of operation; (ii) integrating operational and maintenance policies aspects for both production and utility units; (iii) considering startup and shutdown related decisions and constraints for both production and utility unit; (iv) modeling modified maximum operating levels for units that are under online condition-based cleaning; and (v) providing an optimization framework that can be readily used within a rolling horizon scheme to cope with unexpected events (e.g., fluctuations in demands for products or unit breakdowns). To the best of our knowledge, this is the first work that deals with the problem under consideration and provides such an integrated framework for its solution. Of great importance is also the fact that in this study comprehensive comparisons are made between the solutions obtained following the proposed integrated approach and the traditional sequential approach, demonstrating clearly the important benefits of the proposed approach over its sequential counterpart. Overall, the proposed integrated method follows a whole-system approach that addresses the efficient energy generation, use and consumption (i.e., production and utility units under performance degradation and recovery), improved material handling (i.e., resource-constrained cleaning policies), and integrated management of energy and material resources in dynamic environments (i.e., integrated approach under uncertainties) towards a cleaner and sustainable production in process industries.

\section{Problem Statement}

This work focuses on the detailed condition-based operational and cleaning planning of production and utility systems under alternative resource-constrained cleaning policies, 
through the consideration of performance degradation and recovery for utility and production units. This integrated planning problem is formally defined in terms of the following items:

- A given planning horizon divided into a number of equally-length time periods $t \in T$.

- A set of energy or material resources $e \in E$ that are classified to final product ( $e \in E^{P R}$ ) and utility resources $\left(e \in E^{U T}\right)$. The final products have known demand profiles $\zeta_{(e, t)}$.

- A set of units $i \in I$ that could produce a number of resources $e \in E_{i}$. These units are categorized to utility $\left(i \in U T_{i}\right)$ and production $\left(i \in P R_{i}\right)$ units. Maximum (minimum) operating levels $\kappa_{(i, t)}^{\max }\left(\kappa_{(i, t)}^{\min }\right)$ for utility units and production levels $\bar{\kappa}_{(i, e, t)}^{\max }\left(\bar{\kappa}_{(i, e, t)}^{\min }\right)$ for production units are known. For the units that have a maximum runtime $\left(i \in M R_{i}\right)$, the maximum runtime $\left(o_{i}\right)$ after its last startup is defined. For every unit that is subject to startup and shutdown actions $\left(i \in I^{S F}\right)$, the startup $\left(\phi_{(i, t)}^{S}\right)$ and shutdown $\left(\phi_{(i, t)}^{F}\right)$ costs are also given. For any unit that is subject to minimum runtime and shutdown time restrictions (i.e., $i \in I^{S-\min }$ and $i \in I^{F-\min }$, respectively), the minimum runtime after its last startup $\omega_{i}$ and the minimum idle time after its last shutdown $\psi_{i}$ are also defined.

- A set of resource-dedicated inventory tanks $z \in Z_{e}$ that can receive resources from units $i \in I_{z}^{+}$and send resources to units $i \in I_{z}^{-}$. The inventory tanks have a given maximum (minimum): inventory tank level $\beta_{(e, z)}^{\max }\left(\beta_{(e, z)}^{\min }\right)$, inlet resource flow $\beta_{(e, z, t)}^{+, \max }\left(\beta_{(e, z, t)}^{+, \min }\right)$, and outlet utility resource flow $\beta_{(e, z, t)}^{-, \max }\left(\beta_{(e, z, t)}^{-, \min }\right)$. Initial inventory tank levels $\tilde{\beta}_{(e, z)}$ and losses coefficients $\beta_{z}^{\text {loss }}$ are also given.

- Different cleaning policies for the units are considered. In particular, a unit could be subject to: (i) flexible time-window offline cleaning $\left(i \in F M_{i}\right)$ with a given earliest $\tau_{i}^{e s}$ and latest $\tau_{i}^{l s}$ starting time, (ii) in-progress offline cleaning carried over from the previous 
planning horizon $\left(i \in D M_{i}\right)$, or (iii) condition-based cleaning $\left(i \in C B_{i}\right)$ with known performance degradation rates. Two types of condition-based cleaning tasks are considered, namely: online cleaning tasks $\left(C B_{i}^{o n}\right)$ with given recovery factors $\rho_{i}^{r e c}$, and offline cleaning tasks $\left(C B_{i}^{\text {off }}\right)$.

- A set of alternative cleaning tasks options $q \in Q_{i}$ for each unit that is subject to flexible time-window cleaning $\left(i \in F M_{i}\right)$ or offline condition-based cleaning $\left(i \in C B_{i}^{\text {off }}\right)$. The cleaning tasks options are characterized by different durations $v_{(i, q)}$, cleaning resource requirements $\vartheta_{(i, q)}^{\text {off }}$, and associated cleaning costs $\phi_{(i, q, t)}^{\text {off }}$.

- For every production unit $i \in I_{e}^{P R}$, fixed and variable utility requirements for the production of final products are given $\left(\bar{\alpha}_{\left(i, e, e^{\prime}\right)}\right.$ and $\alpha_{\left(i, e, e^{\prime}\right)}$, respectively).

- Given variable and fixed operating costs for production and utility units, $\phi_{(i, e, t)}^{P R, p \text {-var }}$ and $\phi_{(i, e, t)}^{P R, o p-f i x}$, and $\phi_{(i, t)}^{U T, o p-v a r}$ and $\phi_{(i, t)}^{U T, o p-f i x}$, respectively.

- Given purchase prices for acquiring utility and product resources from external sources, $\phi_{(e, i, t)}^{U T, e x}$ and $\phi_{(e, t)}^{P R, e x}$ respectively.

- A given time-varying energy price profile $\phi_{(i, t)}^{p w}$.

Some additional considerations of the problem under study are the following: (i) the demands for final products should be fully satisfied; and (ii) there is a limited amount of available resources for cleaning tasks per time period.

For every time period, the key decisions to be made by the optimization model are:

- the operational status for each production and utility unit (i.e., startup, shutdown, in operation, idle, under cleaning);

- the operating level for each production and utility unit;

- the inventory level for each inventory tank of utility and product resources; 
- the utility requirements of each production unit; and

- the selection of the timing and the types of the cleaning tasks to be performed in each production and utility unit.

And all these with the goal to minimize the cost of the overall process system which includes:

- fixed and variable operating costs for production and utility units;

- $\quad$ startup and shutdown costs for production and utility units;

- extra energy costs due to performance degradation for production and utility units;

- cleaning costs for production and utility units; and

- $\quad$ penalties or costs for acquiring utility and product resources from external sources.

\section{Optimization Framework}

In this section, a linear mixed integer programming model is presented for the integrated planning problem considered in this study. The proposed mathematical model follows a rolling horizon modelling representation in order to readily deal with various types of uncertainty, such as fluctuations on the demand for final products, unit breakdowns, variations of cost terms, or data inaccuracies. In brief, in the rolling horizon scheme, a planning problem is solved for a certain length of time horizon (i.e., prediction horizon), and then the solution for a part of that time horizon (i.e., control horizon) is executed (typically for the first time period of the prediction horizon). After each iteration, a new planning problem is solved by moving forward the time horizon by the length of the control horizon considered. Figure 2 displays a representative rolling horizon example for the reactive planning problem described above. In a rolling horizon framework, the state of the overall system and the uncertain parameters of the problem are updated before each iteration. The main parameters that need to be updated are: (i) the level of every inventory tank; (ii) the cumulative time of operation per unit; (iii) the deviation of the operating level per unit; (iv) the current operating status of each unit; (v) the startup and shutdown history of units; (vi) the online cleaning history of units; and (vii) the 
demands for products. Figure 3 shows a schematic representative of the steps of the proposed reactive planning method. A description of the proposed optimization framework follows.

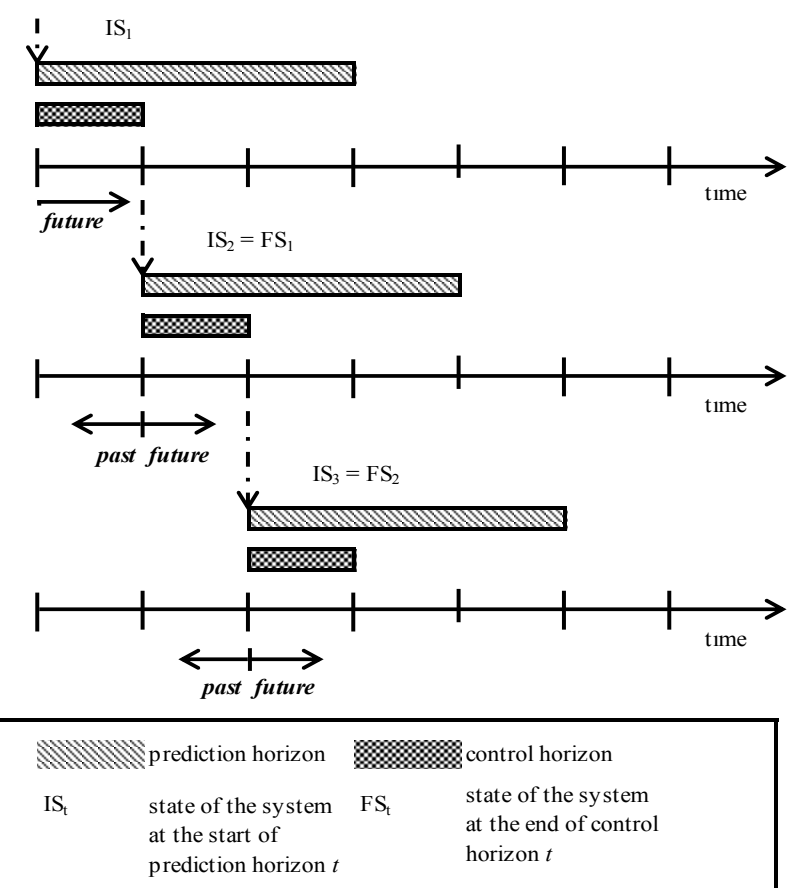

Figure 2. A representative rolling horizon example for reactive planning. 


\section{Initialization Step}

Define the length of the (i) total planning horizon (TH); (ii) prediction horizon $(\mathrm{PH})$; (iii) control horizon $(\mathrm{CH})$; and (iv) the initial state of the system.

Calculate the total number of iterations:

total $=(\mathrm{TH}-\mathrm{PH}+\mathrm{CH}) / \mathrm{CH})$. Set iter $=1$

\section{Update Step}

Update the uncertain parameters (e.g. demands for products) and the current state of the overall system.

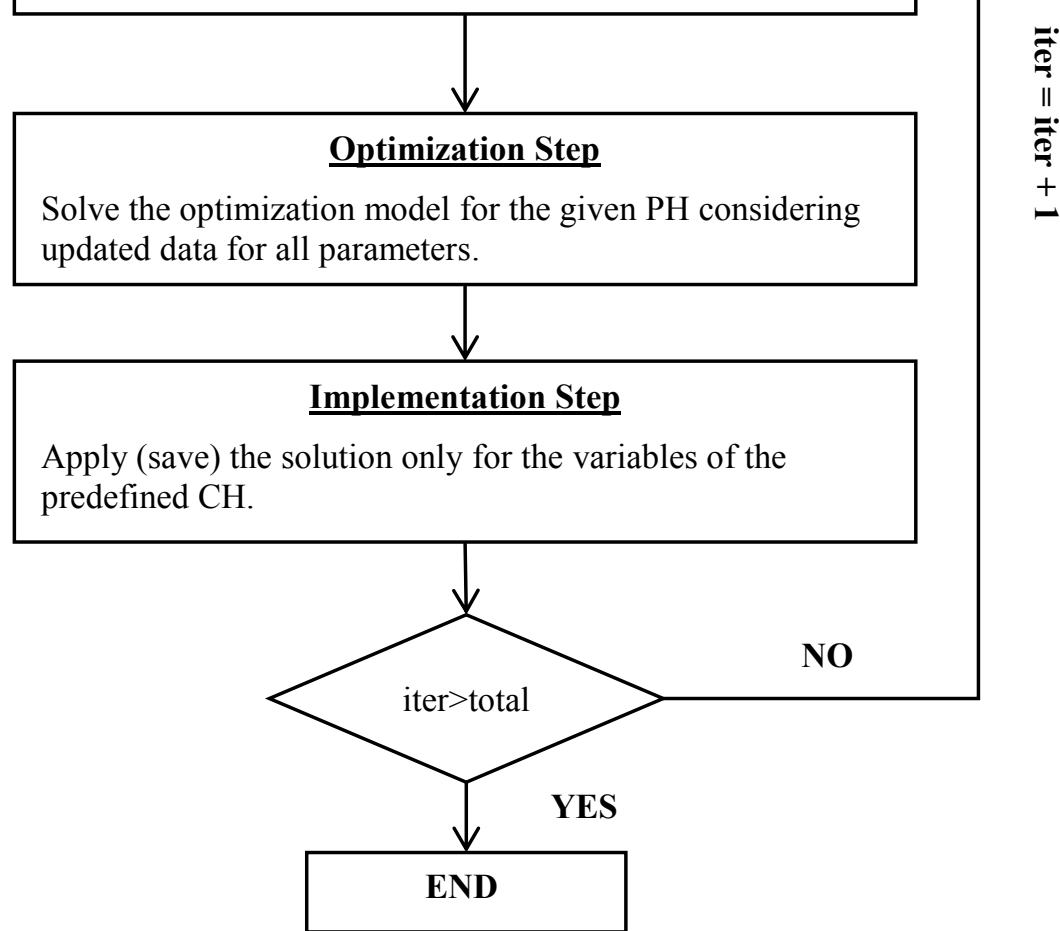

Figure 3. Reactive planning method via rolling horizon.

\subsection{Startup and Shutdown Actions.}

In order to model the major operational status (i.e., in operation, idle, startup, or shutdown) of production and utility units, the following set of binary variables is introduced:

$X_{(i, t)}=\left\{\begin{array}{l}1 \text { if unit } i \text { is operating during time period } t \\ 0 \text { otherwise. }\end{array}\right.$

$S_{(i, t)}=\left\{\begin{array}{l}1 \text { if unit } i \text { starts up at the beginning of time period } t \\ 0 \text { otherwise. }\end{array}\right.$

$F_{(i, t)}=\left\{\begin{array}{l}1 \text { if unit } i \text { shuts down at the beginning of time period } t \\ 0 \text { otherwise. }\end{array}\right.$ 
The operational status of each unit is then modeled according to:

$$
\begin{array}{ll}
S_{(i, t)}-F_{(i, t)}=X_{(i, t)}-\tilde{\chi}_{i} & \forall i \in I^{S F}, t \in T: t=1 \\
S_{(i, t)}-F_{(i, t)}=X_{(i, t)}-X_{(i, t-1)} & \forall i \in I^{S F}, t \in T: t>1 \\
S_{(i, t)}+F_{(i, t)} \leq 1 & \forall i \in I^{S F}, t \in T
\end{array}
$$

The first two sets of constraints relate the startup and shutdown actions with the operating binary variables, while the last set of constraints ensure that no startup and shutdown action can occur simultaneously.

The minimum runtime $\omega_{i}$ and shutdown time $\psi_{i}$ for any unit subject to minimum runtime or shutdown restriction are modeled by constraints (2) and (3), respectively.

$$
\begin{aligned}
& X_{(i, t)} \geq \sum_{t^{\prime}=\max \left\{1, t-\omega_{i}+1\right\}}^{t} S_{\left(i, t^{\prime}\right)} \quad \forall i \in I^{S-m i n}, t \in T: \omega_{i}>1 \\
& X_{(i, t)}=1 \quad \forall i \in I^{S-m i n}, t=1, \ldots,\left(\omega_{i}-\tilde{\omega}_{i}\right): 0<\tilde{\omega}_{i}<\omega_{i} \\
& 1-X_{(i, t)} \geq \sum_{t^{\prime}=\max \left\{1, t-\psi_{i}+1\right\}}^{t} F_{\left(i, t^{\prime}\right)} \quad \forall i \in I^{F-\min }, t \in T: \psi_{i}>1 \\
& X_{(i, t)}=0 \quad \forall i \in I^{F-\min }, t=1, \ldots,\left(\psi_{i}-\tilde{\psi}_{i}\right): 0<\tilde{\psi}_{i}<\psi_{i}
\end{aligned}
$$

Parameters $\tilde{\omega}_{i}\left(\tilde{\psi}_{i}\right)$ describe the initial state of each unit with respect to its total number of consecutive operating (idle) periods since its last startup (shutdown) at the beginning of the current planning horizon. Constraints (2) and (3) are needed only if the minimum runtime $\omega_{i}$ or shutdown time $\psi_{i}$ of a unit is greater than a single time period, respectively.

Generally speaking, a maximum runtime $\left(o_{i}\right)$ may be imposed for units $i \in M R_{i}$ that do not follow a more detailed performance-based cleaning planning, according to:

$$
\begin{array}{ll}
\sum_{t^{\prime}=\max \left\{1, t-o_{i}\right\}}^{t} X_{\left(i, t^{\prime}\right)} \leq o_{i} & \forall i \in M R_{i}, t \in T \\
\sum_{t^{\prime}=\max \left\{1, t-\left(o_{i}-\tilde{\omega}_{i}\right)\right\}}^{t} X_{\left(i, t^{\prime}\right)} \leq\left(o_{i}-\tilde{\omega}_{i}\right) & \forall i \in M R_{i}, t=\left(o_{i}-\tilde{\omega}_{i}+1\right): \tilde{\omega}>1
\end{array}
$$




\subsection{Cleaning Tasks.}

As discussed in Problem Statement, the different unit cleaning policies considered are: (i) flexible time-window offline cleaning $\left(i \in F M_{i}\right.$ ), (ii) in-progress offline cleaning carried over from the previous planning horizon $\left(i \in D M_{i}\right)$, or (iii) condition-based cleaning $\left(i \in C B_{i}\right)$. Online cleaning $\left(C B_{i}^{o n}\right)$ and offline cleaning tasks $\left(C B_{i}^{\text {off }}\right)$ are considered for the conditionbased cleaning. The following binary variables are defined to model these cleaning tasks.

$H_{(i, q, t)}=\left\{\begin{array}{l}1 \text { if a cleaning task option } q \text { for } i \in\left(C B_{i}^{\text {off }} \cup F M_{i}\right) \text { begins at the start of time period } t \\ 0 \text { otherwise. }\end{array}\right.$ $W_{(i, t)}=\left\{\begin{array}{l}1 \text { if an offline cleaning task for } i \in\left(C B_{i}^{\text {off }} \cup F M_{i}\right) \text { begins at the start of time period } t \\ 0 \text { otherwise. }\end{array}\right.$ $V_{(i, t)}=\left\{\begin{array}{l}1 \text { if an online cleaning task for } i \in\left(C B_{i}^{o n} \cap U T_{i}\right) \text { takes place in time period } t \\ 0 \text { otherwise. }\end{array}\right.$ $V_{(i, e, t)}^{P R}=\left\{\begin{array}{l}1 \text { if an online cleaning task for } i \in\left(C B_{i}^{o n} \cap P R_{i}\right) \text { that produces product } e \in E_{i} \text { in time period } t, \\ 0 \text { otherwise. }\end{array}\right.$

\subsubsection{In-progress offline cleaning tasks.}

At the beginning of the planning horizon, there may be some in-progress unfinished offline cleaning tasks for some units $\left(i \in D M_{i}\right)$ which are carried over from the previous planning horizon. These cleaning tasks are modeled according to:

$$
X_{(i, t)}=0 \quad \forall i \in D M_{i}, t \in T: \tilde{\eta}_{(i, t)}>0
$$

Parameters $\tilde{\eta}_{(i, t)}$ represent the known cleaning resources requirements of units that are under in-progress offline cleaning at the beginning of the planning horizon of interest.

\subsubsection{Flexible time-window offline cleaning tasks.}

In general, there may be alternative options for these offline cleaning tasks. And as such, one cleaning task option need to start within the given time window $t=\left[\tau_{i}^{e s}, \tau_{i}^{l s}\right]$, as given by: 
$\sum_{q \in Q_{i} t=\tau_{i}^{e s}} \sum_{(i, q, t)}^{\tau_{i}^{k s}}=1 \quad \forall i \in F M_{i}$

Observe that multiple such cleaning tasks can be modeled for a unit by providing different nonoverlapping time windows, if needed.

\subsubsection{Condition-based online cleaning tasks.}

In any given time period, a unit could be under online cleaning only if the unit is under operation during this period, as modeled by:

$V_{(i, t)} \leq X_{(i, t)} \quad \forall i \in C B_{i}^{o n}, t \in T$

In practice very frequent online cleaning may affect negatively the condition and operation of a unit. For this reason, the proposed approach considers that a unit can undergo an online cleaning task after a minimum time period has passed from the occurrence of the previous online cleaning task in the same unit, as given by:

$\begin{array}{ll}\sum_{t^{\prime}=\max \left\{1, t-\gamma_{i}^{o n}+1\right\}}^{t} V_{\left(i, t^{\prime}\right)} \leq 1 & \forall i \in C B_{i}^{o n}, t \in T \\ V_{(i, t)}=0 & \forall i \in C B_{i}^{o n}, t \leq\left(\gamma_{i}^{o n}-\tilde{\gamma}_{i}^{o n}\right): \tilde{\gamma}_{i}^{o n}<\gamma_{i}^{o n}\end{array}$

Parameters $\tilde{\gamma}_{i}^{o n}$ and $\gamma_{i}^{o n}$ represent the total number of time periods that has passed since the last online cleaning at the beginning of the planning horizon and the minimum time between two consecutive online cleaning tasks in a unit, respectively.

$V_{(i, t)}=\sum_{e \in E_{i}} V_{(i, e, t)}^{P R} \quad \forall i \in\left(C B_{i}^{o n} \cap P R_{i}\right), t \in T$

Constraints (9) relate the two binary variables for online cleaning tasks for the production units.

These constraints are needed in order to model correctly the modified maximum operating levels of production units during the period that are under online cleaning. If online cleaning does not affect the maximum operating level of production units, then these constraints can be ignored and variables $V_{(i, e, t)}^{P R}$ do not need to be defined.

\subsubsection{Condition-based cleaning tasks: unit performance degradation and recovery.}


In this study, the performance of any unit that is subject to condition-based maintenance is modeled through the extra energy consumption of the unit $U_{(i, t)}$ due to its deviation from its completely clean condition (i.e., full performance). The performance of the unit decreases as the extra energy consumption increases. To avoid the energy inefficient use and potential damage of the unit, this extra energy consumption for the units under operation should not exceed a maximum extra energy consumption limit $v_{i}^{\max }$, according to:

$U_{(i, t)} \leq v_{i}^{\max } X_{(i, t)} \quad \forall i \in C B_{i}, \forall t \in T$

To continue with, the extra energy consumption of an operating unit is related to: (i) its cumulative time of operation $R_{(i, t)}$, and (ii) its cumulative operating level deviation $D_{(i, t)}$ from its reference operating level (where additional energy consumption is considered minimal), as given by:

$\begin{array}{lr}U_{(i, t)} \geq \delta_{i} R_{(i, t)}+\delta_{i}^{q} D_{(i, t)}-v_{i}^{\max }\left(1-X_{(i, t)}\right) & \forall i \in C B_{i}, \forall t \in T \\ U_{(i, t)} \leq \delta_{i} R_{(i, t)}+\delta_{i}^{q} D_{(i, t)}+v_{i}^{\max }\left(1-X_{(i, t)}\right) & \forall i \in C B_{i}, \forall t \in T\end{array}$

Parameters $\delta_{i}$ and $\delta_{i}^{q}$ represent the degradation rates due to the cumulative time of operation and the deviation from the reference operating level, respectively. In industrial applications, it is significant to take into consideration the extra energy consumption contribution due to operation out of the reference operating level since this affects the condition of the equipment. Figure 4 presents an illustrative example of two alternative operating level profiles of two units that produce the same product. Observe that the two solutions are equivalent in terms of total production level in any time period. On one hand, the first solution shows many operating level fluctuations and most importantly reports operating levels that are far away from the reference operating level (i.e., this implies additional energy consumption). On the other hand, the second solution reports operating levels for both units equal to the reference operating level in all time periods (i.e., all $D_{(i, t)}$ are zero). In other words, although the two solutions are equivalent in 
terms of total production, the smooth operation of the second solution results in reduced extra energy consumption and thus slower performance degradation of the unit.



(a) Solution 1: units with different operating levels
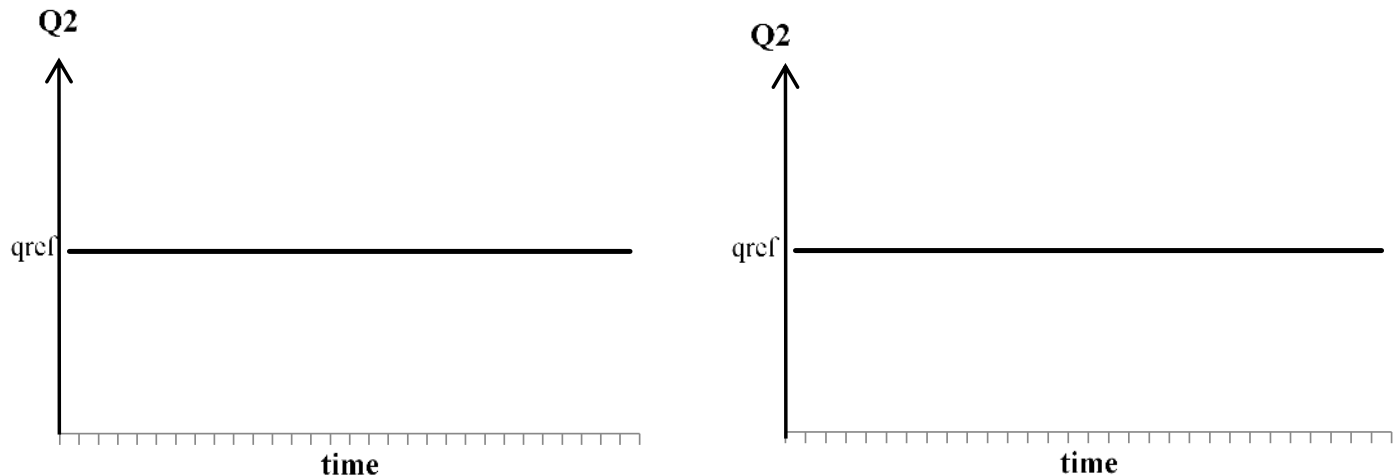

(b) Solution 2: units with same operating levels.

Figure 4. Illustrative example for operating level deviation of the units.

\section{Cumulative time of operation:}

The occurrence of an offline cleaning task in a unit resets its cumulative time of operation to zero, according to:

$R_{(i, t)} \leq \bar{\mu}_{(i, t)}\left(1-W_{(i, t)}\right) \quad \forall i \in C B_{i}^{o f f}, \forall t \in T$

Parameters $\bar{\mu}_{(i, t)}$ are sufficient big numbers. Good values for these parameters for each unit can be calculated through the corresponding maximum extra energy consumption and degradation rate parameters. 
The cumulative time of operation for a unit subject to condition-based cleaning is modeled by the following set of constraints:

$$
\begin{array}{ll}
R_{(i . t)} \leq\left(\tilde{\rho}_{i}+X_{(i . t)}\right)+\bar{\mu}_{(i . t)}\left(W_{(i . t)}+V_{(i . t)}\right) & \forall i \in C B_{i}, \forall t \in T: t=1 \\
R_{(i . t)} \leq\left(R_{(i . t-1)}+X_{(i . t)}\right)+\bar{\mu}_{(i . t)}\left(W_{(i . t)}+V_{(i . t)}\right) & \forall i \in C B_{i}, \forall t \in T: t>1 \\
R_{(i . t)} \geq\left(\tilde{\rho}_{i}+X_{(i . t)}\right)-\bar{\mu}_{(i . t)}\left(W_{(i . t)}+V_{(i . t)}\right) & \forall i \in C B_{i}, \forall t \in T: t=1 \\
R_{(i . t)} \geq\left(R_{(i . t-1)}+X_{(i . t)}\right)-\bar{\mu}_{(i . t)}\left(W_{(i . t)}+V_{(i . t)}\right) & \forall i \in C B_{i}, \forall t \in T: t>1 \\
R_{(i . t)} \geq\left(\tilde{\rho}_{i}+1\right)\left(1-\rho_{i}^{r e c}\right)-\bar{\mu}_{(i . t)}\left(1-V_{(i . t)}\right) & \forall i \in C B_{i}^{o n}, \forall t \in T: t=1 \\
R_{(i . t)} \geq\left(R_{(i . t-1)}+1\right)\left(1-\rho_{i}^{r e c}\right)-\bar{\mu}_{(i . t)}\left(1-V_{(i . t)}\right) & \forall i \in C B_{i}^{o n}, \forall t \in T: t>1
\end{array}
$$

For every unit, parameter $\rho_{i}^{\text {rec }}$ represents the corresponding performance recovery factor due to its online cleaning and parameter $\tilde{\rho}_{i}$ denotes the cumulative time of operation just before the beginning of the planning horizon of interest (i.e., initial state). Notice that a unit could be subject to both offline and online condition-based cleaning tasks in the proposed approach.

\section{Cumulative operating level deviation:}

Similarly to the cumulative time of operation, the occurrence of an offline cleaning task in a unit resets its cumulative operating level deviation to zero, according to:

$$
D_{(i, t)} \leq \mu_{(i, t)}\left(1-W_{(i, t)}\right) \quad \forall i \in C B_{i}^{o f f}, \forall t \in T
$$

Parameters $\mu_{(i, t)}$ are sufficient big numbers that could be calculated through the corresponding maximum extra energy consumption and degradation rate parameters.

For a utility unit subject to condition-based cleaning, the cumulative operating level deviation from its reference operating level $\left(q_{(i, t)}^{r e f}\right)$ is modeled by the following set of constraints:

$$
\begin{gathered}
D_{(i, t)} \leq \tilde{\rho}_{i}^{d q}+\left(\frac{\left|q_{(i, t)}^{r e f}-\bar{Q}_{(i, t)}\right|}{q_{(i, t)}^{r e f}}\right)+\mu_{(i, t)}\left(W_{(i, t)}+V_{(i, t)}\right)+\mu_{(i, t)}\left(1-X_{(i, t)}\right) \\
\forall i \in\left(C B_{i} \cap U T_{i}\right), t \in T: t=1 \\
D_{(i, t)} \leq \mathrm{D}_{(i, t-1)}+\left(\frac{\mid q_{(i, t)}^{r e f}-\bar{Q}_{(i, t)}}{q_{(i, t)}^{r e f}}\right)+\mu_{(i, t)}\left(W_{(i, t)}+V_{(i, t)}\right)+\mu_{(i, t)}\left(1-X_{(i, t)}\right) \\
\forall i \in\left(C B_{i} \cap U T_{i}\right), t \in T: t>1
\end{gathered}
$$




$$
\begin{aligned}
& D_{(i, t)} \geq \tilde{\rho}_{i}^{d q}+\left(\frac{\left|q_{(i, t)}^{r e f}-\bar{Q}_{(i, t)}\right|}{q_{(i, t)}^{r e f}}\right)-\mu_{(i, t)}\left(W_{(i, t)}+V_{(i, t)}\right)-\mu_{(i, t)}\left(1-X_{(i, t)}\right) \\
& \forall i \in\left(C B_{i} \cap U T_{i}\right), t \in T: t=1 \\
& D_{(i, t)} \geq \mathrm{D}_{(i, t-1)}+\left(\frac{\left|q_{(i, t)}^{r e f}-\bar{Q}_{(i, t)}\right|}{q_{(i, t)}^{r e f}}\right)-\mu_{(i, t)}\left(W_{(i, t)}+V_{(i, t)}\right)-\mu_{(i, t)}\left(1-X_{(i, t)}\right) \\
& \forall i \in\left(C B_{i} \cap U T_{i}\right), t \in T: t>1 \\
& D_{(i, t)} \geq\left(\tilde{\rho}_{i}^{d q}+\left(\frac{\left|q_{(i, t)}^{r e f}-\bar{Q}_{(i, t)}\right|}{q_{(i, t)}^{r e f}}\right)\right)\left(1-\rho_{i}^{r e c}\right)-\mu_{(i, t)}\left(1-V_{(i, t)}\right) \quad \forall i \in\left(C B_{i}^{o n} \cap U T_{i}\right), t \in T: t=1 \\
& D_{(i, t)} \geq\left(\mathrm{D}_{(i, t-1)}+\left(\frac{\left|q_{(i, t)}^{r e f}-\bar{Q}_{(i, t)}\right|}{q_{(i, t)}^{r e f}}\right)\right)\left(1-\rho_{i}^{r e c}\right)-\mu_{(i, t)}\left(1-V_{(i, t)}\right) \quad \forall i \in\left(C B_{i}^{o n} \cap U T_{i}\right), t \in T: t>1
\end{aligned}
$$

For a production unit subject to condition-based cleaning, the cumulative operating level deviation from its reference production level $\left(q_{(i, e, t)}^{r e f}\right)$ is modeled by the following set of constraints:

$$
\begin{aligned}
& D_{(i, t)} \leq \tilde{\rho}_{i}^{q}+\left(\frac{\left|q_{(i, e, t)}^{r e f}-Q_{(i, e, t)}\right|}{q_{(i, e, t)}^{r e f}}\right)+\mu_{(i, t)}\left(W_{(i, t)}+V_{(i, t)}\right)+\mu_{(i, t)}\left(1-X_{(i, t)}\right) \\
& \forall i \in\left(C B_{i} \cap P R_{i}\right), e \in E_{i}, t \in T: t=1 \\
& D_{(i, t)} \leq \mathrm{D}_{(i, t-1)}+\left(\frac{\left|q_{(i, e, t)}^{r e f}-Q_{(i, e, t)}\right|}{q_{(i, e, t)}^{r e f}}\right)+\mu_{(i, t)}\left(W_{(i, t)}+V_{(i, t)}\right)+\mu_{(i, t)}\left(1-X_{(i, t)}\right) \\
& \forall i \in\left(C B_{i} \cap P R_{i}\right), e \in E_{i}, t \in T: t>1 \\
& D_{(i, t)} \geq \tilde{\rho}_{i}^{q}+\left(\frac{\left|q_{(i, e, t)}^{r e f}-Q_{(i, e, t)}\right|}{q_{(i, e, t)}^{r e f}}\right)-\mu_{(i, t)}\left(W_{(i, t)}+V_{(i, t)}\right)-\mu_{(i, t)}\left(1-X_{(i, t)}\right) \\
& \forall i \in\left(C B_{i} \cap P R_{i}\right), e \in E_{i}, t \in T: t=1 \\
& D_{(i, t)} \geq \mathrm{D}_{(i, t-1)}+\left(\frac{\left|q_{(i, e, t)}^{r e f}-Q_{(i, e, t)}\right|}{q_{(i, e, t)}^{r e f}}\right)-\mu_{(i, t)}\left(W_{(i, t)}+V_{(i, t)}\right)-\mu_{(i, t)}\left(1-X_{(i, t)}\right) \\
& \forall i \in\left(C B_{i} \cap P R_{i}\right), e \in E_{i}, t \in T: t>1
\end{aligned}
$$




$$
\begin{gathered}
D_{(i, t)} \geq \tilde{\rho}_{i}^{q}+\left(\frac{\left|q_{(i, e, t)}^{r e f}-Q_{(i, e, t)}\right|}{q_{(i, e, t)}^{r e f}}\right)\left(1-\rho_{i}^{r e c}\right)-\mu_{(i, t)}\left(1-V_{(i, t)}\right) \\
\forall i \in\left(C B_{i}^{o n} \cap P R_{i}\right), e \in E_{i}, t \in T: t=1 \\
D_{(i, t)} \geq \mathrm{D}_{(i, t-1)}+\left(\frac{\left|q_{(i, e, t)}^{r e f}-Q_{(i, e, t)}\right|}{q_{(i, e, t)}^{r e f}}\right)\left(1-\rho_{i}^{r e c}\right)-\mu_{(i, t)}\left(1-V_{(i, t)}\right) \\
\forall i \in\left(C B_{i}^{\text {on }} \cap P R_{i}\right), e \in E_{i}, t \in T: t>1
\end{gathered}
$$

For every unit, parameter $\tilde{\rho}_{i}^{q}$ represents its cumulative operating level deviation just before the beginning of the planning horizon of interest (i.e., initial state).

\subsubsection{Operational constraints for offline cleaning tasks.}

The following set of constraints ensure that a unit that is under offline cleaning remains closed for the whole duration of the selected offline cleaning task option, and relate the two binary variables for offline cleaning tasks.

$$
X_{(i, t)}+\sum_{t^{\prime}=\max \left\{\tau_{i}^{e s}, t-v_{(i, q)}+1\right\}}^{\min \left\{\tau_{i}^{l s}, t\right\}} H_{\left(i, q, t^{\prime}\right)} \leq 1 \quad \forall i \in\left(F M_{i} \cup C B_{i}^{o f f}\right), q \in Q_{i}, \tau_{i}^{e s} \leq t \leq\left(\tau_{i}^{l s}+v_{(i, q)}-1\right)
$$

$W_{(i, t)}=\sum_{q \in Q_{i}} H_{(i, q, t)} \forall i \in\left(F M_{i} \cup C B_{i}^{o f f}\right), t \in T: \tau_{i}^{e s} \leq t \leq \tau_{i}^{l s}$

For condition-based offline cleaning tasks, earliest and latest starting times should be set equal to the first and the last period of the planning horizon, respectively.

\subsubsection{Resource constraints for cleaning tasks.}

In the same line with our previous work (Zulkafli and Kopanos, 2016), a limited amount of available resources for cleaning operations shared by all types of cleaning tasks is considered, according to:

$$
\begin{aligned}
& \sum_{i \in C B_{i}^{o n}} \vartheta_{i}^{o n} V_{(i, t)}+\sum_{i \in C B_{i}^{o f f}} \sum_{q \in Q_{i} t^{\prime}=t-v_{(i, q)}+1}^{t} \vartheta_{(i, q)}^{o \text { off }} H_{\left(i, q, t^{\prime}\right)}+\sum_{i \in F M_{i}} \sum_{q \in Q_{i} t^{\prime}=\max } \sum_{\left\{\tau_{i}^{e s}, t-v_{(i, q)}+1\right\}}^{\min \left\{\tau_{i}^{l s}, t\right\}} \vartheta_{(i, q)}^{o f f} H_{\left(i, q, t^{\prime}\right)} \\
& \leq \eta_{t}^{\max }-\sum_{i \in D M_{i}} \tilde{\eta}_{(i, t)} \quad \forall t \in T
\end{aligned}
$$


For every unit, parameters $\vartheta_{i}^{o n}$ and $\vartheta_{(i, q)}^{\text {off }}$ denote the resource requirements for online cleaning and different offline cleaning task options, respectively.

\subsection{Utility and Product Resources.}

\subsubsection{Utility system: operating level bounds.}

The utility system consists of a number of utility units that could generate the whole set of utility resources required by the production system. If a utility unit operates, its operating level should be between its lower and upper operating level bounds $\left(\kappa_{(i, t)}^{\min }\right.$ and $\left.\kappa_{(i, t)}^{\max }\right)$. Here, changes in the maximum operating levels during online cleaning periods are considered and modeled through: (i) the binary variables related to online cleaning, and (ii) parameters $\pi_{i}^{o n}$ that represent the percentage modification on the upper operating level of a unit that is under online cleaning. Hence, the operating bounds of this general case are given by:

$\kappa_{(i, t)}^{\min } X_{(i, t)} \leq \bar{Q}_{(i, t)} \leq \kappa_{(i, t)}^{\max }\left(X_{(i, t)}-\pi_{i}^{o n} V_{(i, t)}\right) \quad \forall i \in\left(U T_{i} \cap C B_{i}^{o n}\right), t \in T$

Notice that parameters $c_{i}^{o n}$ are activated only if there is an online cleaning task for a unit. In the case that there is no effect on the maximum operating level of some units during their online cleaning, the corresponding parameters $\pi_{i}^{o n}$ of these units are set equal to zero. There are some types of utility units, such as combined heat and power units, which generate at the same time more than one utility resources. The generated amount of any utility resource from each utility unit per time period is modeled by:

$Q_{(i, e, t)}=\rho_{(i, e)} \bar{Q}_{(i, t)} \quad \forall i \in U T_{i}, e \in E_{i}, t \in T$

Parameters $\rho_{(i, e)}$ denote the stoichiometry coefficients that relate the operating level of the utility unit with the generated amount of each utility resource type $\left(Q_{(i, e, t)}\right)$ that is cogenerated by the same utility system (e.g., heat to power ratio of a combined heat and power unit).

\subsubsection{Production system: production level bounds.}


The production system consists of a number of production units that produce the whole set of product resources required by the customers. Here, the production process is modeled as singlestage with a number of units operating in parallel. In order to model the production statuses and levels for production units, the following binary variables are introduced:

$Y_{(i, e, t)}=\left\{\begin{array}{l}1 \text { if production unit } i \in \mathrm{PR}_{i} \text { produces product resource } e \text { in time period } t \\ 0 \text { otherwise. }\end{array}\right.$

If a production unit produces a product resource $e$, its production level should be between its lower and upper production level bounds $\left(\bar{\kappa}_{(i, e, t)}^{\max }\right.$ and $\left.\bar{\kappa}_{(i, e, t)}^{\min }\right)$. Similarly to utility units, changes in the maximum production levels during online cleaning periods are considered. Therefore, the production bounds of this general case are given by:

$\bar{\kappa}_{(i, e, t)}^{\min } Y_{(i, e, t)} \leq Q_{(i, e, t)} \leq \bar{\kappa}_{(i, e, t)}^{\max }\left(Y_{(i, e, t)}-\pi_{i}^{o n} V_{(i, e, t)}^{P R}\right) \quad \forall i \in\left(P R_{i} \cap C B_{i}^{o n}\right), e \in E_{i}, t \in T$

Online cleaning, as its name implies, could take place in time periods where production units are on operation, as modeled by:

$V_{(i, e, t)}^{P R} \leq Y_{(i, e, t)} \quad \forall i \in\left(P R_{i} \cap C B_{i}^{o n}\right), e \in E_{i}, t \in T$

The two types of operating binary variables for the production units are related by the following set of constraints:

$$
\begin{array}{ll}
Y_{(i, e, t)} \leq X_{(i, t)} & \forall i \in P R_{i}, e \in E_{i}, t \in T \\
X_{(i, t)} \leq \sum_{e \in E_{i}} Y_{(i, e, t)} \leq 1 & \forall i \in P R_{i}, \forall t \in T
\end{array}
$$

According to these constraints, operating binary variables $X_{(i, t)}$ would be equal to one if and only if there is production of a product resource. In addition, the latter constraints ensure that a production unit could produce at most one product resource per time period.

\subsubsection{Inventory tanks.}

Production and utility systems contain a number of resource-dedicated inventory tanks. These inventory tanks can receive resources $\left(B_{(e, z, t)}^{+}\right)$from their associated units $I_{z}$, according to: 
$B_{(e, z, t)}^{+}=\sum_{i \in\left(I_{e} \cap I_{z}\right)} Q_{(i, e, t)} \quad \forall e \in E, z \in Z_{e}, t \in T$

Lower and upper bounds on the inlet flows of resources to inventory tanks are considered by:

$\varepsilon_{(e, z, t)}^{+, \min } \leq B_{(e, z, t)}^{+} \leq \varepsilon_{(e, z, t)}^{+, \max } \quad \forall e \in E, z \in Z_{e}, t \in T$

Resource balances for every resource-dedicated inventory tank per time period are given by:

$$
\begin{array}{ll}
B_{(e, z, t)}=\tilde{\beta}_{(e, z)}+B_{(e, z, t)}^{+}-B_{(e, z, t)}^{-} & \forall e \in E, z \in Z_{e}, t \in T: t=1 \\
B_{(e, z, t)}=\left(1-\beta_{z}^{\text {loss }}\right) B_{(e, z, t-1)}+B_{(e, z, t)}^{+}-B_{(e, z, t)}^{-} & \forall e \in E, z \in Z_{e}, t \in T: t>1
\end{array}
$$

Notice that variables $B_{(e, z, t)}$ indicate the inventory level per resource and inventory tank at the end of each time period and variables $B_{(e, z, t)}^{-}$represent the outlet resource flow from each inventory tank. Parameters $\tilde{\beta}_{(e, z)}$ stand for the initial inventory for each resource inventory tank at the beginning of the planning horizon (i.e., initial state) and parameters $\beta_{z}^{\text {loss }}$ provide the losses coefficients for each resource inventory tank. Minimum and maximum inventory levels for the inventory tanks are also considered as:

$\xi_{(e, z)}^{\min } \leq B_{(e, z, t)} \leq \xi_{(e, z)}^{\max } \quad \forall e \in E, z \in Z_{e}, t \in T$

The amount of each utility resource that leaves its dedicated inventory tank and its minimum and outlet flows are given by the following set of constraints:

$$
\begin{aligned}
& B_{(e, z, t)}^{-}=\sum_{i \in\left(P R_{i} \cap I_{z}\right)} B_{(e, z, i, t)}^{U T,-} \quad \forall e \in E^{U T}, z \in Z_{e}, t \in T \\
& \varepsilon_{(e, z, t)}^{-, \min } \leq B_{(e, z, t)}^{-} \leq \varepsilon_{(e, z, t)}^{-, \max } \quad \forall e \in E^{U T}, z \in Z_{e}, t \in T
\end{aligned}
$$

\subsubsection{Demands for product resources.}

The demands for final products $\left(\zeta_{(e, t)}\right)$ should be satisfied for every time period, according to:

$$
N S_{(e, t)}^{F P}+\sum_{z \in Z_{e}} B_{(e, z, t)}^{-}=\zeta_{(e, t)} \quad \forall e \in E^{P R}, t \in T
$$

Variables $N S_{(e, t)}^{F P}$ denote the amount of the demand for each product resource $\left(E^{P R}\right)$ per time period that cannot be satisfied by the internal production system. These unsatisfied demands 
for product resources should be covered by acquiring product resources from external sources. Generally speaking, this is highly undesirable and for this reason a very high penalty or purchase cost is usually used in the optimization goal. If product resources cannot be acquired from external sources, variables $N S_{(e, t)}^{F P}$ present the lost sales of product resources.

\subsubsection{Demands for utility resources (link between utility and production systems).}

The requirements for utility resources give the linking constraints between utility and production systems. For each time period, the demands for utility resources per production unit $I_{e}^{P R}$ consist of: (i) fixed utility resource requirements that depend on the operational status of the production unit; and (ii) variable utility resource requirements that depend on the production level of the production unit.

$$
N S_{(e, i, t)}^{U T}+\sum_{z \in\left(Z_{e} \cap Z_{i}\right)} B_{(e, z, i, t)}^{U T,-}=\sum_{e^{\prime} \in\left(E^{P R} \cap E_{i}\right)}\left(\alpha_{\left(i, e, e^{\prime}\right)} Q_{\left(i, e^{\prime}, t\right)}+\bar{\alpha}_{\left(i, e, e^{\prime}\right)} Y_{\left(i, e^{\prime}, t\right)}\right) \quad \forall e \in E^{U T}, i \in I_{e}^{P R}, t \in T
$$

Variables $N S_{(e, i, t)}^{U T}$ represent the amount of unsatisfied demand for each utility resource per time period. Similarly to the unsatisfied demand for product resources, penalty or purchase costs for acquiring utility resources from external sources are typically introduced in the objective function of the optimization problem.

\subsection{Objective Function}

The optimization goal is to minimize the total cost of the production and the utility system. More specifically, the objective function includes: (i) startup and shutdown costs for units that are subject to startup and shutdown actions; (ii) variable and fixed operating costs for utility units; (iii) variable and fixed production costs for production units; (iv) penalty or purchase costs for acquiring product and utility resources from external sources; (v) total extra energy consumption costs for utility and production units that are subject to performance degradation modeling; and (vi) total cleaning costs related to online and offline cleaning tasks of production and utility units that are subject to performance degradation. The optimization goal is given by: 


$$
\min \left[\begin{array}{l}
\sum_{t \in T} \sum_{i \in I^{S F}}\left(\phi_{(i, t)}^{S} S_{(i, t)}+\phi_{(i, t)}^{F} F_{(i, t)}\right)+\sum_{t \in T} \sum_{i \in I^{U T}}\left(\phi_{(i, t)}^{U T, o p-v a r} \bar{Q}_{(i, t)}+\phi_{(i, t)}^{U T, o p-f i x} X_{(i, t)}\right) \\
+\sum_{t \in T} \sum_{i \in P R_{i}} \sum_{e \in E_{i}}\left(\phi_{(i, e, t)}^{P R, o p-v a r} Q_{(i, e, t)}+\phi_{(i, e, t)}^{P R, o p-f i x} Y_{(i, e, t)}\right) \\
+\sum_{t \in T} \sum_{e \in E^{P R}} \phi_{(e, t)}^{P R, e x} N S_{(e, t)}^{F P}+\sum_{t \in T} \sum_{e \in E^{U T}} \sum_{i \in I_{P R}^{e}} \phi_{(e, i, t)}^{U T, e x} N S_{(e, i, t)}^{U T} \\
+\sum_{t \in T} \sum_{i \in C B_{i}} \phi_{(i, t)}^{p w} U_{(i, t)}+\sum_{t \in T}\left(\sum_{i \in C B_{i}^{o n}} \phi_{(i, t)}^{o n} V_{(i, t)}+\sum_{i \in\left(C B_{i}^{o f f} \cup F M_{i}\right)} \sum_{q \in Q_{i}} \phi_{(i, q, t)}^{o f f} H_{(i, q, t)}\right)
\end{array}\right]
$$

In the above expression, the small-letter symbols correspond to the associated cost coefficients of the corresponding optimization variables. A detailed definition of them can be found in the Nomenclature.

\subsection{Remarks on Rolling Horizon}

Terminal constraints should be defined for some key optimization variables when a rolling horizon approach is used. These constraints are applied for the last time period $|T|$ of the considered prediction horizon and can be typically related to desired minimum resource inventory levels or unit performance levels, as modeled below:

$$
\begin{array}{ll}
B_{(e, z, t)} \geq \lambda_{(e, z)}^{B} \xi_{(e, z)}^{\max } & \forall e \in E, z \in Z_{e}, t \in T: t=|T| \\
U_{(i, t)} \leq \lambda_{i}^{U} v_{i}^{\max } & \forall i \in C B_{i}, t \in T: t=|T|
\end{array}
$$

Parameters $\lambda_{(e, z)}^{B}$ and $\lambda_{i}^{U}$ represent are percentage coefficients used to determine the minimum inventory level for each resource and the maximum extra energy consumption level for each operating unit at the last period of each prediction horizon. In the same line, terminal constraints could be defined for other variables if needed. Generally speaking, terminal constraints are defined as a mean of preserving the stability of the system over its long-term operational horizon. It is also usual to apply terminal constraint values even in deterministic optimization approaches, in order to ensure a better state of the system at the end of the planning horizon. More details about rolling horizon appraches can be found in Kopanos and Pistikopoulos (2014). 


\section{Case Studies.}

In this part, three case studies for the integrated planning problem of utility and production systems are presented in order to highlight the special features of the proposed optimization framework. More specifically, the first case study studies only a flexible time-window cleaning policy for units while the second case study considers both flexible time-window and condition-based cleaning policies for production and utility units. The third case study deals with the reactive planning problem under a rolling horizon approach and considers conditionbased cleaning policies for all units. All case studies have been solved following both the proposed integrated approach and the traditional sequential approach. Detailed comparisons between the solutions of both approaches have been made. All resulting optimization problems have been solved in GAMS/CPLEX in an Intel(R) core(TM) i7 under standard configurations and a zero optimality gap.

\subsection{Case Study 1: Integrated Planning of Utility and Production Systems (Flexible Time-Window Cleaning).}

In this case study, flexible time-window offline cleaning tasks for utility and production units are only considered (i.e., no condition-based maintenance). All parameters are deterministic.

\subsubsection{Description of Case Study 1}

The system under consideration consists of five utility units ( $i 1-i 5)$ and three production units (i6-i8). The utility units can produce two utility resources $(e 1, e 2)$ which could be either stored in their associated inventory tanks $(z 1, z 2)$ or consumed directly by the production units. Two final product resources $(e 3, e 4)$ can be produced by the production units that can be either stored in their dedicated inventory tanks $(z 3, z 4)$ or meet directly the customer demand. Each utility and production unit has a maximum operating level, as given by Table 1 . Minimum operating levels for units are $10 \%$ of the corresponding maximum operating levels. For each production unit and product resource, Table 2 provides the stoichiometric coefficients 
of fixed and varied utility needs for the production of a unit of the associated product resource. Table 3 gives the cogeneration coefficient of each utility resource for every utility units. For example, for utility unit $i 1$, four units of $e 2$ are generated for every unit of $e 1$ produced. Notice that utility unit i4 and i5 cannot generate utility resource $e 2$ and $e 1$, respectively. Maximum runtimes for units are not considered. There is a maximum number of available resources for cleaning tasks equal to 12 cleaning resource units. The minimum runtime for utility and production units $\left(\omega_{i}\right)$ is 6 days and the minimum offline time after shutdown $\left(\psi_{i}\right)$ is 3 days. No lower bounds are considered for minimum inventory level $\left(\xi_{(e, z)}^{\min }\right)$, minimum flows of resources to inventory tanks $\left(\varepsilon_{(e, z, t)}^{+, \min }\right)$ and minimum flows of resources leaves inventory tanks $\left(\varepsilon_{(e, z, t)}^{-, \min }\right)$. There is no maximum resources flow constraint to inventory tank $\left(\varepsilon_{(e, z, t)}^{+, \max }\right)$. The maximum inventory level $\left(\xi_{(e, z)}^{\max }\right)$ for resources $e 1, e 2, e 3$, and $e 4$ are 100, 320, 200 and 300 units, respectively. The maximum flows of utility resources leaving their respective inventory $\operatorname{tank}\left(\varepsilon_{(e, z, t)}^{-, \max }\right)$ are 400 units for utility resource $e 1$ and 600 units for utility resource $e 2$.

Table 1. Case Study 1: Maximum operating levels for utility and production units.

\begin{tabular}{lllllllll}
\hline$\kappa_{(i, e, t)}^{\max }$ & $i 1$ & $i 2$ & $i 3$ & $i 4$ & $i 5$ & $i 6$ & $i 7$ & $i 8$ \\
\hline$e 1$ & 50 & 80 & 60 & 60 & - & - & - & - \\
$e 2$ & 200 & 160 & 180 & - & 140 & - & - & - \\
$e 3$ & - & - & - & - & - & 85 & 65 & 50 \\
$e 4$ & - & - & - & - & - & 65 & 50 & 85 \\
\hline
\end{tabular}

Table 2. Case Study 1: Fixed and varied stoichiometric coefficients of utility needs for production units (per unit of product resource).

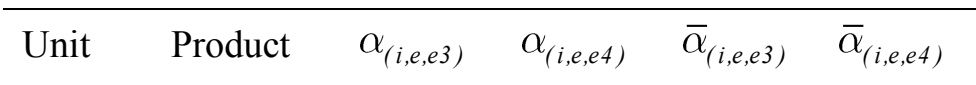




\begin{tabular}{llllll}
\hline \multirow{2}{*}{$i 6$} & $e 1$ & 0.90 & 0.80 & 17 & 15 \\
& $e 2$ & 2.25 & 3.38 & 45 & 39 \\
\hline \multirow{2}{*}{$i 7$} & $e 1$ & 0.80 & 0.70 & 14 & 18 \\
& $e 2$ & 3.38 & 5.25 & 54 & 30 \\
\hline \multirow{2}{*}{$i 8$} & $e 1$ & 0.75 & 0.90 & 16 & 10 \\
& $e 2$ & 2.63 & 3.00 & 36 & 48 \\
\hline
\end{tabular}

Table 3. Case Study 1: Cogeneration coefficients of utility units per utility resource.

\begin{tabular}{lll}
\hline$\rho_{(i, e)}$ & $e 1$ & $e 2$ \\
\hline$i 1$ & 1 & 4 \\
$i 2$ & 1 & 2 \\
$i 3$ & 1 & 3 \\
$i 4$ & 1 & 0 \\
$i 5$ & 0 & 1 \\
\hline
\end{tabular}

A total planning horizon of 30 days, divided in day time periods (i.e., 30 time periods), is considered. All utility and production units should undergo a flexible time-window offline cleaning tasks. The earliest/latest cleaning startup times $\left(\tau_{i}^{e s} / \tau_{i}^{l s}\right)$ are on day 9 and 15 for utility units and on day 20 and 25 for production units, respectively. There are three alternative flexible time-window offline cleaning options $(q 1, q 2, q 3)$ that are characterized by different durations, cleaning resources requirements and associated costs, as shown in Table 4. Operational costs for utility and production units are given in Table 5. Purchase costs for utility and product resources are 6,000 and 4,000 m.u./unit, respectively.

Table 4. Case Study 1: Alternative options for flexible time-window offline cleaning tasks.

\begin{tabular}{llllll}
\hline units & parameter & metric unit & $q 1$ & $q 2$ & $q 3$
\end{tabular}




\begin{tabular}{llllll}
\hline$i 1-i 8$ & $\nu_{(i, q)}$ & days & 3 & 4 & 5 \\
$i 1-i 8$ & $\vartheta_{(i, q)}^{\text {off }}$ & resource units & 6 & 4 & 3 \\
$i 1, i 2, i 5-i 8$ & $\phi_{(i, q, t)}^{\text {off }}$ & m.u./cleaning & $2,137.5$ & $1,425.0$ & $1,068.8$ \\
$i 3$ and $i 4$ & $\phi_{(i, q, t)}^{\text {off }}$ & m.u./cleaning & $7,087.5$ & $4,725.0$ & $3,543.8$ \\
\hline
\end{tabular}

Table 5. Case Study 1: Operational costs for utility and production units.

\begin{tabular}{|c|c|c|c|c|c|}
\hline units & resource & $\begin{array}{l}\phi_{(i, t)}^{S} \\
\text { (m.u./unit) }\end{array}$ & $\begin{array}{l}\phi_{(i, t)}^{F} \\
\text { (m.u./unit) }\end{array}$ & $\begin{array}{l}\phi_{(i, e, t)}^{f i x} \\
\text { (m.u./unit) }\end{array}$ & $\begin{array}{l}\phi_{(i, e, t)}^{v a r} \\
\text { (m.u./unit) }\end{array}$ \\
\hline$i 1$ & $e 1 \& e 2$ & 2,300 & 1,150 & 220 & 10 \\
\hline$i 2$ & $e 1 \& e 2$ & 2,350 & 1,170 & 250 & 10 \\
\hline$i 3$ & $e 1 \& e 2$ & 2,370 & 1,200 & 270 & 10 \\
\hline$i 4$ & el & 2,250 & 1,000 & 150 & 15 \\
\hline$i 5$ & $e 2$ & 2,270 & 1,050 & 200 & 15 \\
\hline$i 6$ & $e 3 \mid e 4$ & 2,300 & 1,150 & $500 \mid 400$ & $1.2 \mid 1.0$ \\
\hline$i 7$ & $e 3 \mid e 4$ & 2,000 & 1,100 & $400 \mid 300$ & 1.5 | 1.4 \\
\hline$i 8$ & $e 3 \mid e 4$ & 2,300 & 1,150 & $300 \mid 500$ & $1.4 \mid 1.9$ \\
\hline
\end{tabular}

The initial inventory for resources $e 1, e 2, e 3$ and $e 4$ is 10, 20, 50 and 300 units, respectively. It is assumed that the process plant is closed before the beginning of the planning horizon of interest, therefore there is no initial state (i.e., $\tilde{\chi}_{i}, \tilde{\psi}_{i}$, or $\tilde{\omega}_{i}$ ) that is taken into account for this case study. In addition, Figure 5 shows the normalized demand for product resources by having the peak demand value of product resource $e 4$ as a reference. The range for demand for product resource $e 3$ is between 40 to 100 units and for product resource $e 4$ is between 50 to 120 unit, respectively. 


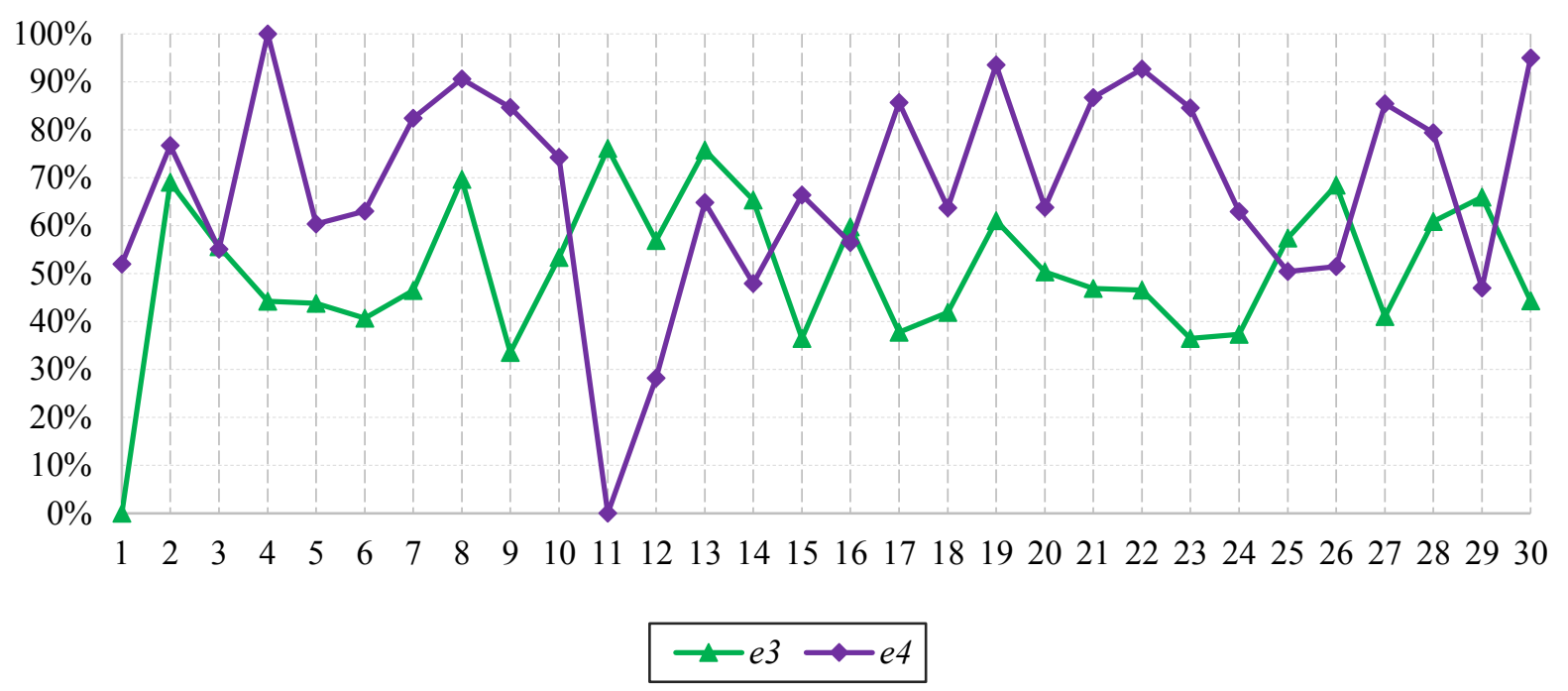

Figure 5. Case study 1: Normalized demand profiles for products per time period.

\subsubsection{Results of Case Study 1 - Integrated Approach}

This example has been solved by using the proposed integrated optimization framework, and the results obtained are reported, analyzed and discussed below.

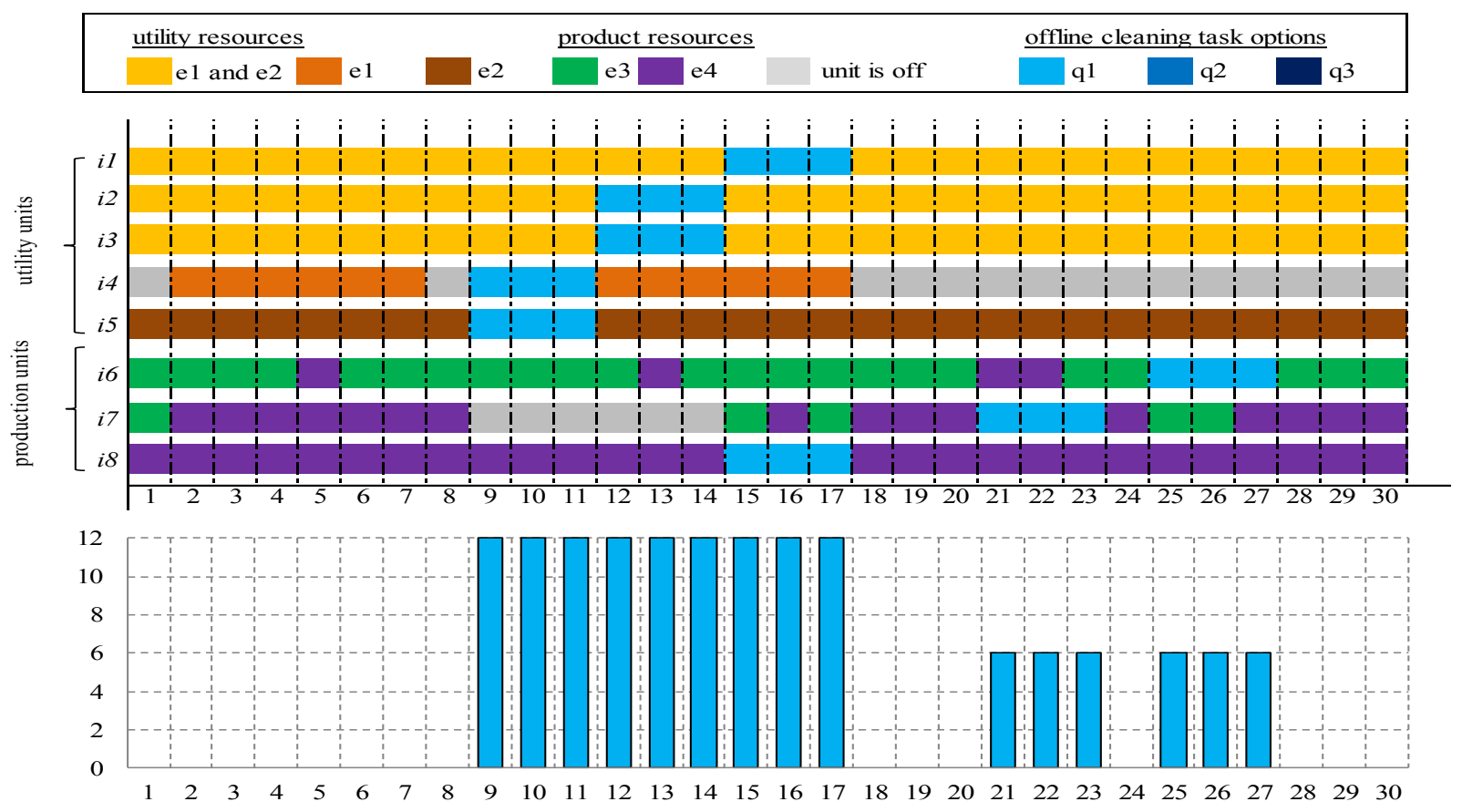

Figure 6. Case Study 1 - Integrated Approach: Optimal operational and cleaning plan for production and utility systems and total utilization profile of cleaning resources. 
Figure 6 displays the optimal operational and cleaning plan for both the utility and the production system. More specifically, this figure shows for each unit per time period: (i) the operational status (i.e., in operation, idle, startup, shutdown, or under cleaning), (ii) the selected offline cleaning task options, (iii) the type of utility or product resources produced from each unit, and (iv) the profile of the cleaning resources requirements. No performance level profiles are displayed in this case study because no condition-based cleaning tasks are considered here.

Simultaneous cleaning tasks between utility units are observed. For instance, utility units $i 4$ and $i 5$ are under cleaning from day 9 to 11 and utility units $i 2$ and $i 3$ are under cleaning from day 12 to 14 . In addition, it is observed a simultaneous cleaning for utility unit $i 1$ and production unit i8 from day 15 to 17 . The flexible time-window for the cleaning of production units is long enough to avoid simultaneous cleaning tasks of multiple production units. Notice that in the optimal solution the most expensive cleaning option $q 1$ (but with the smaller duration) has only been selected most probably because of: (i) the overall high demands for product resources throughout the planning horizon of interest; (ii) the relatively narrow flexible time-windows for the cleaning of utility units; (iii) the constrained availability of cleaning resources per time period; and (iv) the high purchase costs for utility and product resources.

Utility unit $i 4$, which can generate only utility resource $e 1$, is not operating in day 1 and day 8 , because there is enough supply of utility el from the other utility units and its corresponding inventory tank. Production unit $i 7$ is idle from day 9 to 14 mainly due to following two reasons: (i) two utility units are under cleaning during these periods (see Figure 6) a fact that decreases the total utility generation capacity of the plant and therefore the total production capacity as well; and (ii) the total demands for products are relatively lower in these time periods (see Figure 5). 


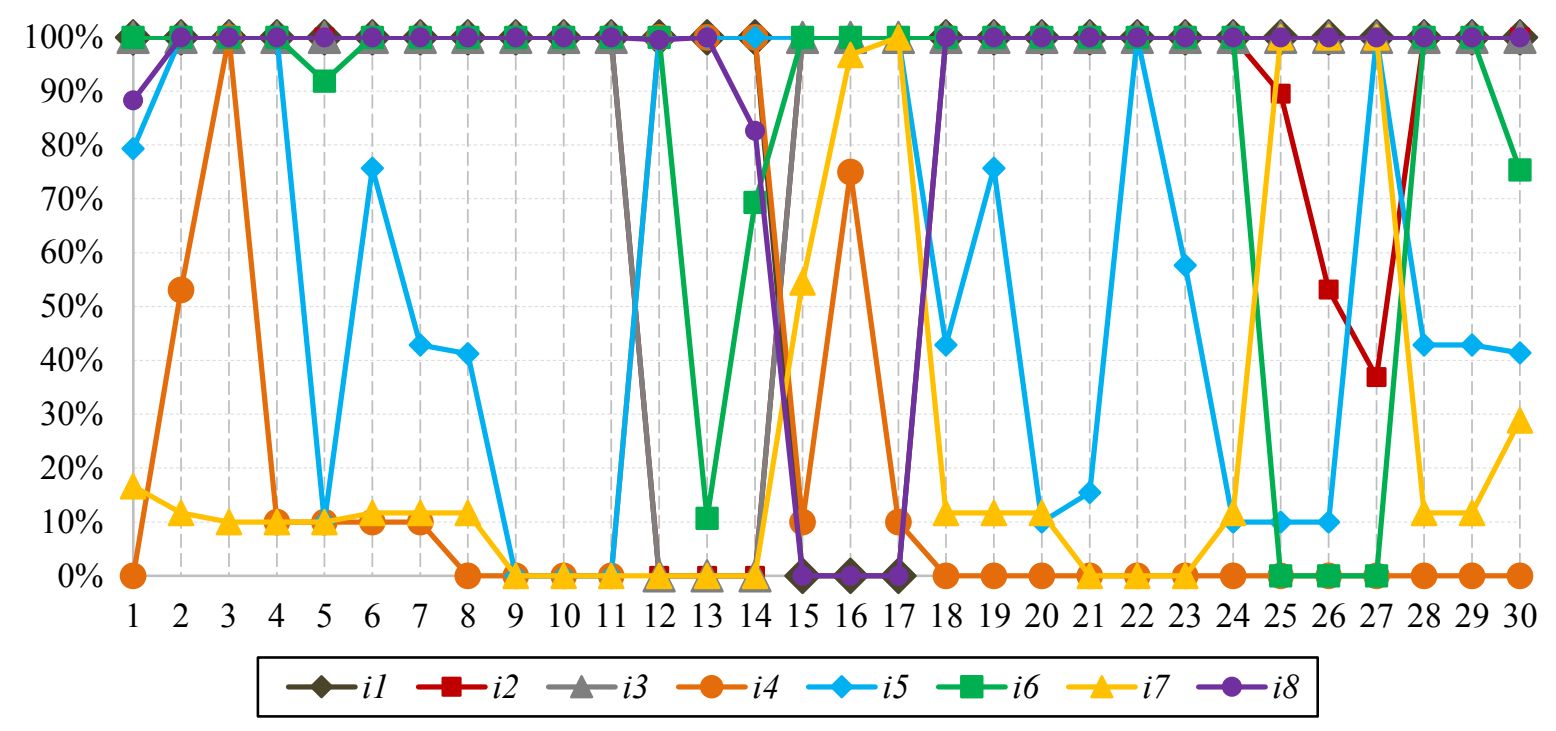

Figure 7. Case Study 1 - Integrated Approach: Normalized operating level profiles for utility and production units.

Figure 7 displays the normalized operating level profiles for all utility and production units. The maximum operating level of each unit has been used as a reference of normalization (see Table 1). In the utility system, utility units $i 1$ to $i 3$ operate at their maximum operating levels throughout the planning horizon (excluding their cleaning periods). It is observed that utility unit $i 4$ that can generate only utility $e 1$ and utility unit $i 5$ that can generate only utility $e 2$ operate in a broader operating range to cover the fluctuations of the utility requirements of the production system. In the production system, production units i6 and is operate at their maximum capacities most of the time periods, while production unit $i 7$ operates at its minimum capacity. The latter is observed basically due to the relatively high shutdown costs compared to fixed and variable operating cost at the minimum operating level. For this reason, it is preferred to continue operating this production unit at minimum capacity and avoid shutting it down, since this would impose a considerable shutdown cost. 


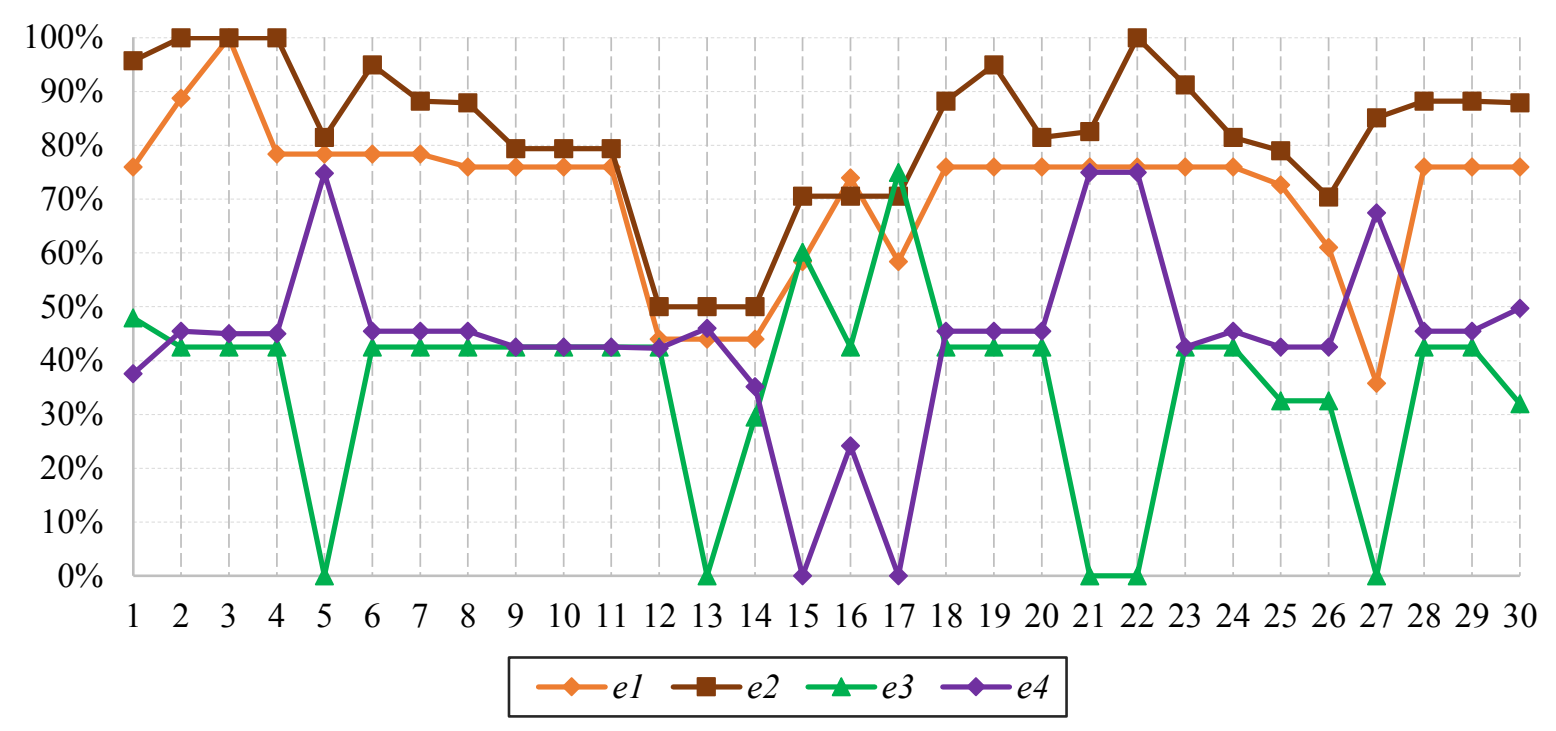

Figure 8. Case Study 1 - Integrated Approach: Normalized total production profiles for utility and final product resources.

Figure 8 displays the normalized total production profiles for every utility and final product resource. The production of each resource is calculated by having the cumulative production of the resource from each unit divided by the maximum total resource capacity of all units. Not surprisingly, it is observed that the trend of the total production profile for $e 3$ follows the opposite trend of that of $e 4$, since the limited number of production units can produce at most one final product per time period. For instance, the high total production peak levels for product resource $e 4$ instead of low total production levels for product $e 3$ in days 5, 13, 21, 22 and 27 are due to the fact that the production units produce exclusively product $e 4$ in all these days (see also Figure 6). The opposite trend is observed in day 15, and 17 when high total peak levels for product e3 but low levels for product e4 when production units produce only product $e 3$ in these days. Meanwhile, the production trends for utilities $e 1$ and $e 2$ follow quite a similar trend throughout the planning horizon, mainly due to the presence of three utility units that cogenerate both utility resources. For example, there is a reduction in the total operating levels for utility resources $e 1$ and $e 2$ when the utility units undergo cleaning between day 9 and 15 . 
Figure 9 displays the normalized inventory profiles for utility and product resources, having as reference the corresponding maximum inventory level of each inventory tank. Low utility inventory levels from day 12 to 20 are mainly due to reduced utility capacities, because utility units $i 1, i 2$ and $i 3$ are under cleaning tasks in this period (see Figure 6). Importantly, there is no purchase of utility or product resources at any time period. From day 20 and onwards, the inventory levels of product resource $e 3$ are low because of: (i) the occurrence of a cleaning task in production unit i6 (see Figure 6); and (ii) its high demands (as shown in Figure 5). Similarly, the low inventory profile for product $e 4$ from day 17 and onwards is due to its higher demand and the cleaning of production unit $i 7$ started in day 21 .

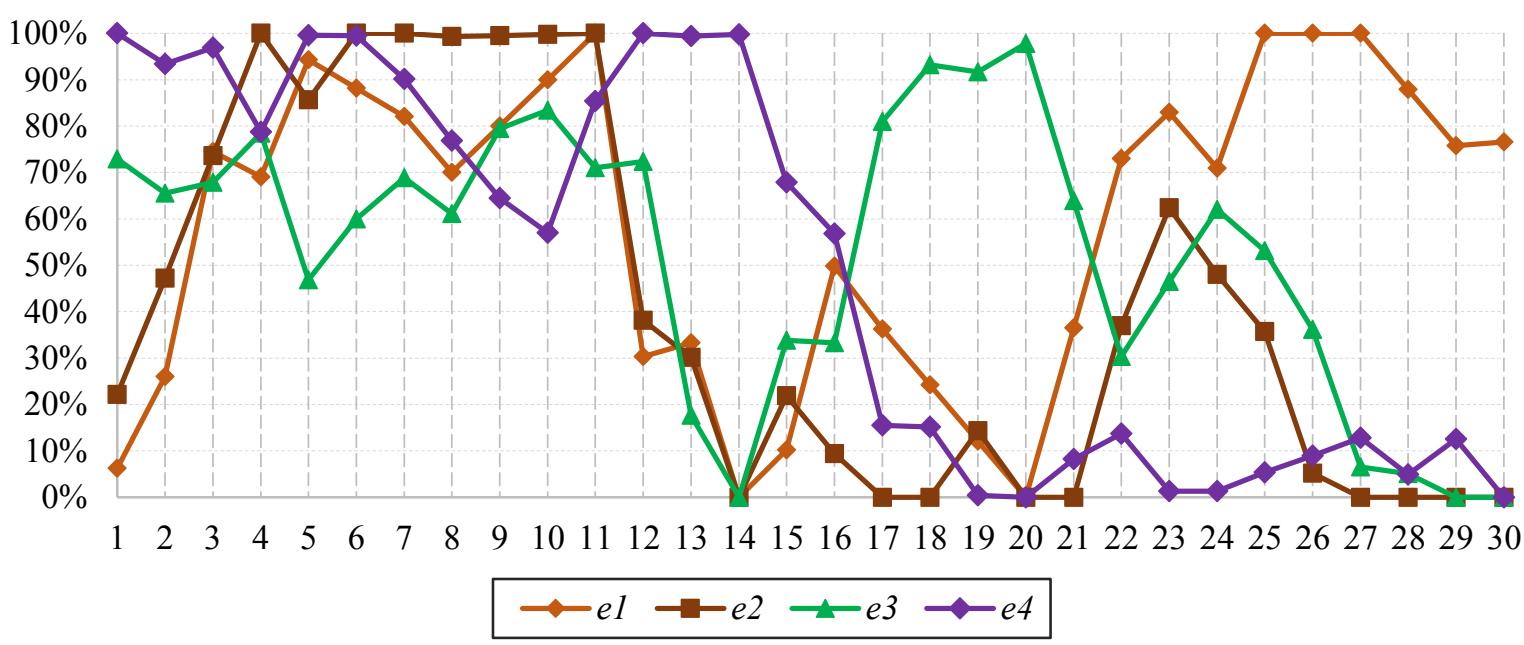

Figure 9. Case Study 1 - Integrated approach: Normalized inventory profiles for utility and product resources. 


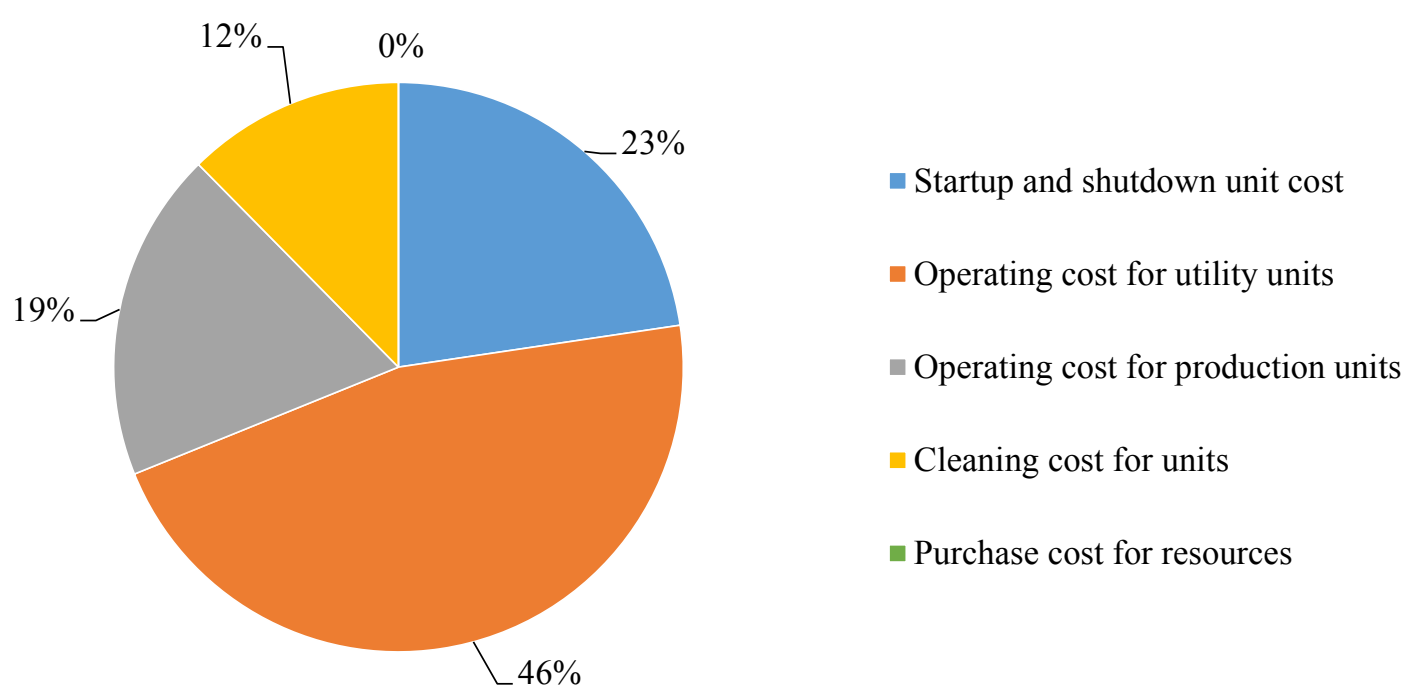

\section{Figure 10. Case study 1 - Integrated Approach: Total cost breakdown (percentage).}

Figure 10 shows the breakdown of the total cost for the utility and the production systems. The costs are divided into: (i) the startup and shutdown operations; (ii) the operation of the utility system; (iii) the operation of the production system; (iv) the offline cleaning tasks for the units; and (v) the total purchase of utility and product resources. The operational cost for the utility system remains the highest cost term at about $46 \%$ of the total cost. The second highest cost is the startup and shutdown units costs which is about $23 \%$ of the total cost, because of the initial state of the overall system (plant was closed before the beginning of the planning horizon). The cleaning cost is around $12 \%$ of the total cost while there is no purchase cost.

\subsubsection{Results of Case Study 1 - Sequential Approach}

Here, the same case study has been solved considering the traditional sequential approach, where the planning problem of the production system is solved first using simply upper bounds on the total available utility amounts per time period. After the solution of this production planning problem, the associated variables that describe the production of final products (i.e., $Q_{(i, t)}$ and $\left.Y_{(i, e, t)}\right)$, product inventories and flows (i.e., $B_{(e, z, t)}, B_{(e, z, t)}^{-}$, and $\left.B_{(e, z, t)}^{+}\right)$and occurrence of cleaning tasks in the production units (i.e., $H_{(i, q, t)}$ ) are fixed, and the planning problem of the utility system is solved. 


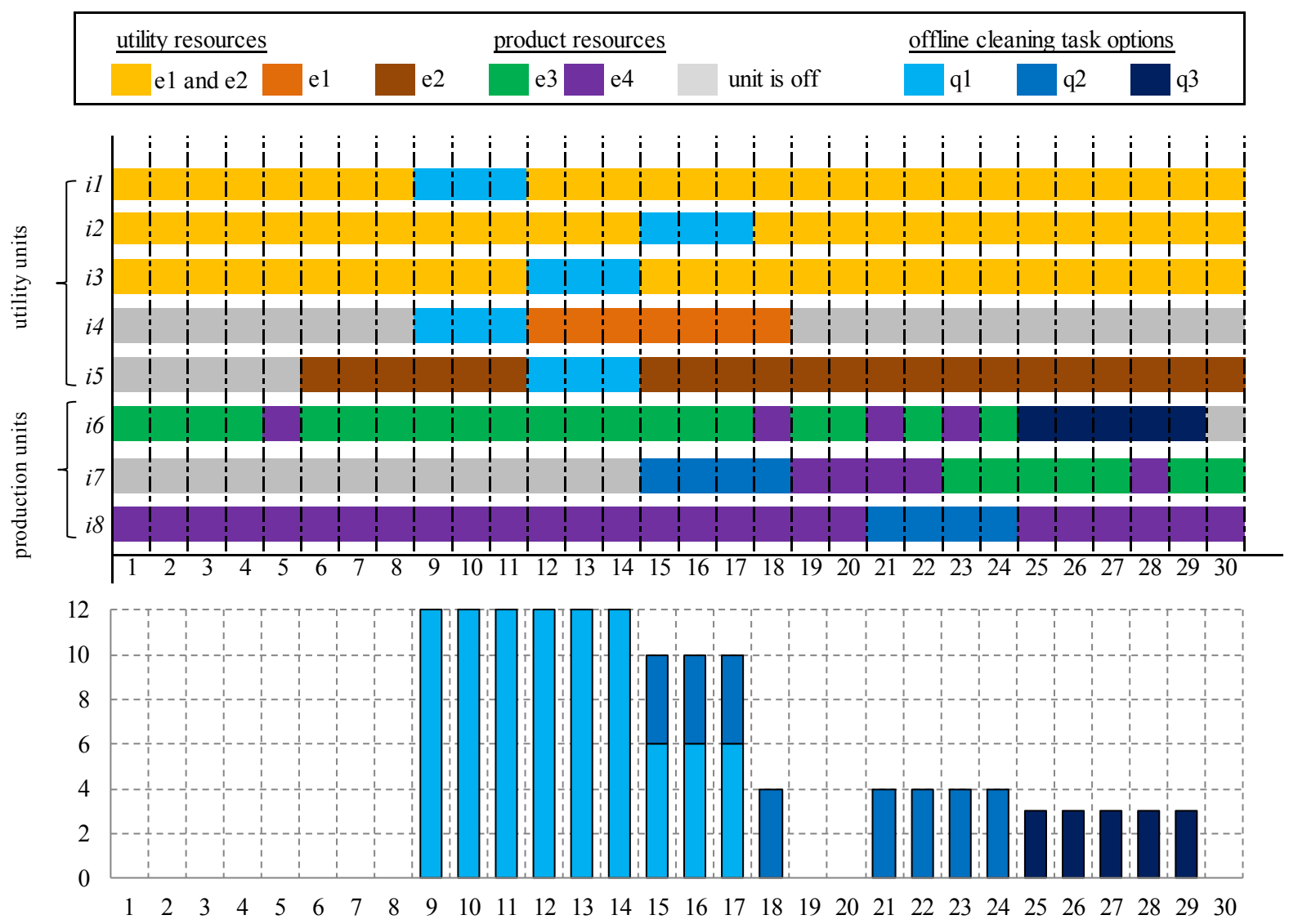

Figure 11. Case Study 1 - Sequential Approach: Operational and cleaning plan for production and utility systems and total utilization profile of cleaning resources.

Figure 11 displays the operational and cleaning plan for the production and the utility system obtained by following the sequential approach. In this case, cleaning tasks options $q 2$ and $q 3$ are selected for the production units. It should be emphasized, in contrast to the solution of the integrated approach, the solution of the sequential approach reports purchases of utilities from external sources in some time periods, as shown in Figure 12. In particular, important utility purchases are observed between day 10 and 16 because of the occurrence of multiple cleaning tasks in the utility units over this time window (see Figure 12). Furthermore, utility units i4 and $i 5$ operate in less time periods in the solution of the sequential approach than in that of the integrated approach which cause the need for utility purchases (see Figure 6). A total of 633 units of utility resources need to be purchased throughout the planning horizon. If there is no 
option of acquiring utilities from externals sources, this would make the production plan infeasible in practice. The total cost of the solution following the integrated approach is more than $5 \%$ lower than that of the solution found by the sequential approach, which is a clear evidence of the benefits that the proposed integrated approach can have over its sequential counterpart.



Figure 12. Case study 1: Sequential approach. Normalized profile of total purchases for utilities.

\subsection{Case Study 2: Integrated Planning of Utility and Production System (Condition- based Cleaning and Flexible Time-Window Cleaning).}

In this case study, a condition-based cleaning policy for utility units and a flexible time-window cleaning policy for production units are considered. The condition-based cleaning policy involves online and offline cleaning tasks. All parameters are deterministic.

\subsubsection{Description of Case Study 2}

Here a modified version of the previous case study is considered. The main parameters (Table 1-4) and operational costs (Table 5) are the same as in Case Study 1. Minimum runtime and shutdown times are the same as in Case Study 1. The demand for products for this case study follows the same pattern as in the previous example, but reduced by $15 \%$. A main difference here is that the utility units (i1-i5) should undergo condition-based cleaning tasks. 
Meanwhile, production unit $i 6$ has a fixed offline cleaning and the other production units ( $i 7$ -i8) should undergo flexible time-window offline cleaning tasks. The earliest and latest cleaning startup times $\left(\tau_{i}^{e s} / \tau_{i}^{l s}\right)$ for production units $i 7$ and $i 8$ are in day 15 and 25, respectively. As before, there are three alternative cleaning tasks options that can be selected for condition-based offline cleaning (i.e., utility units) and time-window flexible cleaning (i.e., production units). The maximum available resources per time period for the cleaning tasks are 12 units of cleaning resources. The parameters that refer to condition-based offline and online cleaning for utility units are defined as follows: (i) the extra power consumption limit $\left(v_{i}^{\max }\right)$; (ii) performance degradation rate $\left(\delta_{i}\right)$; (iii) performance coefficient related to operating level ( $\left.\delta_{i}^{q}\right)$; (iv) minimum time between two consecutive online cleaning tasks $\left(\gamma_{i}^{\text {on }}\right)$; (iv) the recovery factor of the online cleaning for any utility unit $\left(\rho_{i}^{\text {rec }}\right) ;(\mathrm{v})$ references operating level $\left(q_{(i, t)}^{\text {ref }}\right)$; and (iv) the resource requirement of online cleaning $\left(\vartheta_{i}^{o n}\right)$ as shown in Table 6.

Table 6. Case Study 2: Parameters related to the condition-based cleaning of utility units.

\begin{tabular}{llllll}
\hline Parameter & $i 1$ & $i 2$ & $i 3$ & $i 4$ & $i 5$ \\
\hline$v_{i}^{\text {max }}$ & 162 & 153 & 247 & 200 & 210 \\
$\delta_{i}$ & 9 & 9 & 13 & 10 & 10 \\
$\delta_{i}^{q}$ & 6.75 & 6.75 & 9.75 & 7.50 & 7.50 \\
$\gamma_{i}^{\text {on }}$ & 10 & 10 & 10 & 10 & 10 \\
$\rho_{i}^{\text {rec }}$ & 0.20 & 0.20 & 0.20 & 0.20 & 0.20 \\
$q_{(i, t)}^{\text {ref }}$ & 50 & 80 & 60 & 60 & 70 \\
$\vartheta_{i}^{\text {on }}$ & 1 & 1 & 1 & 1 & 1 \\
\hline
\end{tabular}


At the end of the planning horizon of interest, there are two types of terminal constraints for the: (i) inventory levels of utility and product resources; and (ii) the performance level of the operating utility units. Namely, at the end of the planning horizon, the inventory levels of each resource should be greater or equal to $25 \%$ from its corresponding maximum inventory level ( $\left.\xi_{(e, z)}^{\max }\right)$, and the performance level of each utility unit that is under operation at the end of the planning should be greater or equal to $25 \%$ (i.e., lower or equal to $75 \%$ of the corresponding $\left.v_{i}^{\max }\right)$. In addition, Table 7 gives the values of the parameters that define the initial state of the utility and production systems. All other initial state parameters are zero.

Table 7. Case Study 2. Initial state of the utility and production system.

\begin{tabular}{llll}
\hline parameter & $i 1$ & $i 2$ & $i 3$ \\
\hline$\tilde{\rho}_{i}$ & 2 & 4 & 2 \\
$\tilde{\beta}_{(e 1, z 1)}$ & 10 & units & Initial inventory for utility $e 1$ \\
$\tilde{\beta}_{(e 2, z 2)}$ & 20 & units & Initial inventory for utility $e 2$ \\
$\tilde{\beta}_{(e 3, z 3)}$ & 50 & units & Initial inventory for product $e 3$ \\
$\tilde{\beta}_{(e 4, z 4)}$ & 300 & units & Initial inventory for product $e 4$ \\
\hline
\end{tabular}

\subsubsection{Results of Case Study 2 - Integrated Approach}

Figure 13 displays the optimal operational and cleaning plan for both production and utility system. For each production and utility unit: (i) the operational status at each time period; (ii) the selected offline cleaning tasks options and online cleaning tasks on its corresponding time period; (iii) the type of utility or product resources produced from each unit; and (iv) the profile of the cleaning resources requirements are observed. 

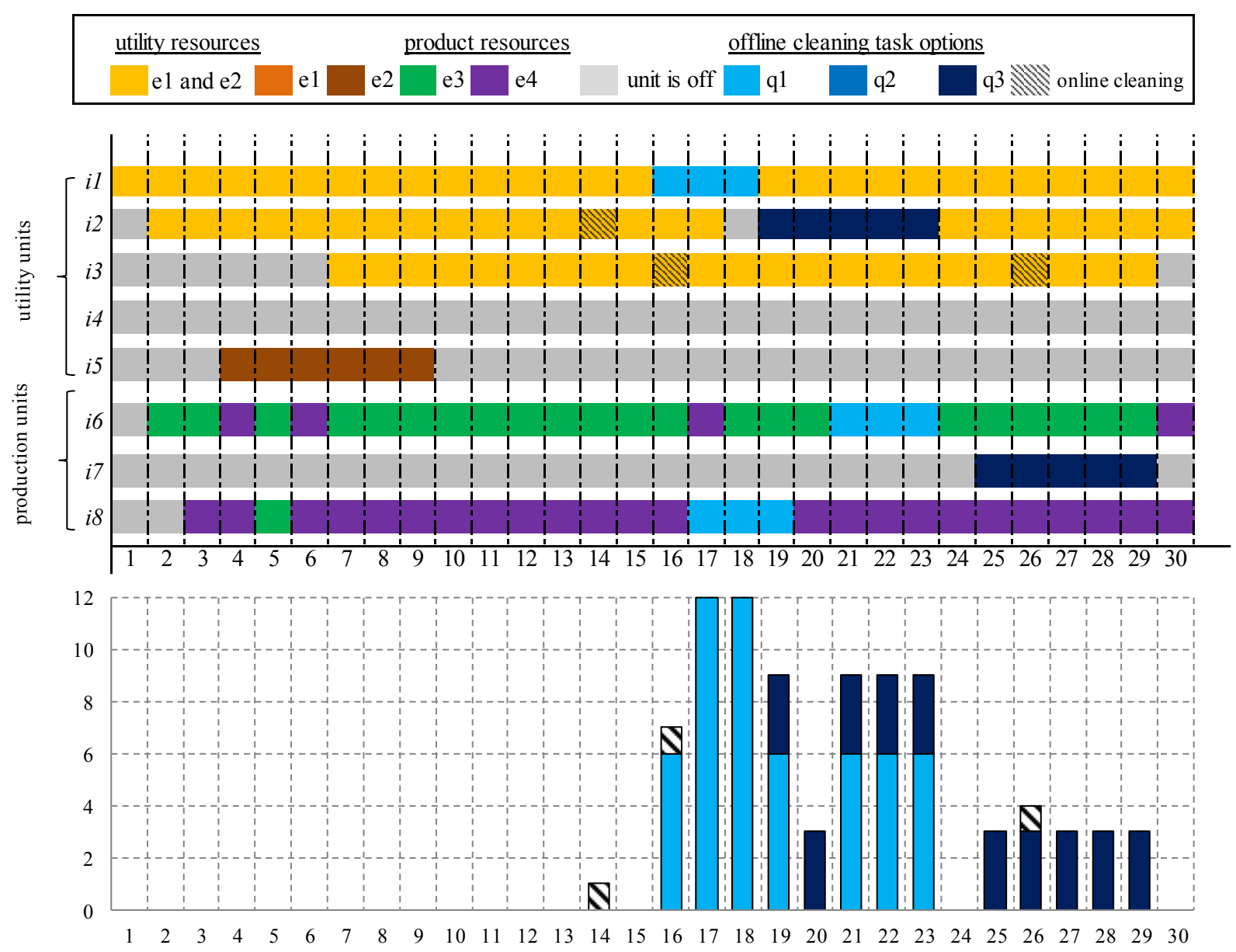

Figure 13. Case Study 2 - Integrated Approach: Optimal operational and cleaning plan

for production and utility systems and total cleaning resources utilization profile.

Simultaneous condition-based offline cleaning tasks are observed for utility unit il and production unit i8 in day 17 and 18 . The solution reports condition-based cleaning tasks for utility units il to $i 3$. Meanwhile, utility unit i4 that can only produce utility el remains closed for all time periods because utility resource $e 1$ has enough supply from other utility units (e.g., $i 1, i 2$ and $i 3$ ) that can cogenerate both utility resources. Utility unit $i 5$ which can only produce utility resource $e 2$ operates in a shorter duration from day 4 to 9 because utility unit $i 3$ is closed. The demand for utility resource $e 2$ cannot be satisfied by just utility unit $i 1$ and $i 2$, thus utility unit $i 5$ operates to fully satisfy this demand in these days. Production unit i6 produces product resource $e 3$ and production unit is produces product resource $e 4$ in 
most of the time periods. This should be due to the stoichiometric coefficient $\alpha_{(i, e e, e)}$ and $\beta_{(i, e e, e)}$ that define the utility requirements per product unit (see Table 2). Another observation is that, production unit $i 7$ remains idle throughout planning horizon but there is a predefined flexible cleaning task option $q 3$ that starts in day 25 . It should clear here that the longest duration cleaning task option is selected due to its lower cost. In reality, the production manager may find that this cleaning is not necessary because this production unit does not operate in the current planning horizon, and may ignore it.

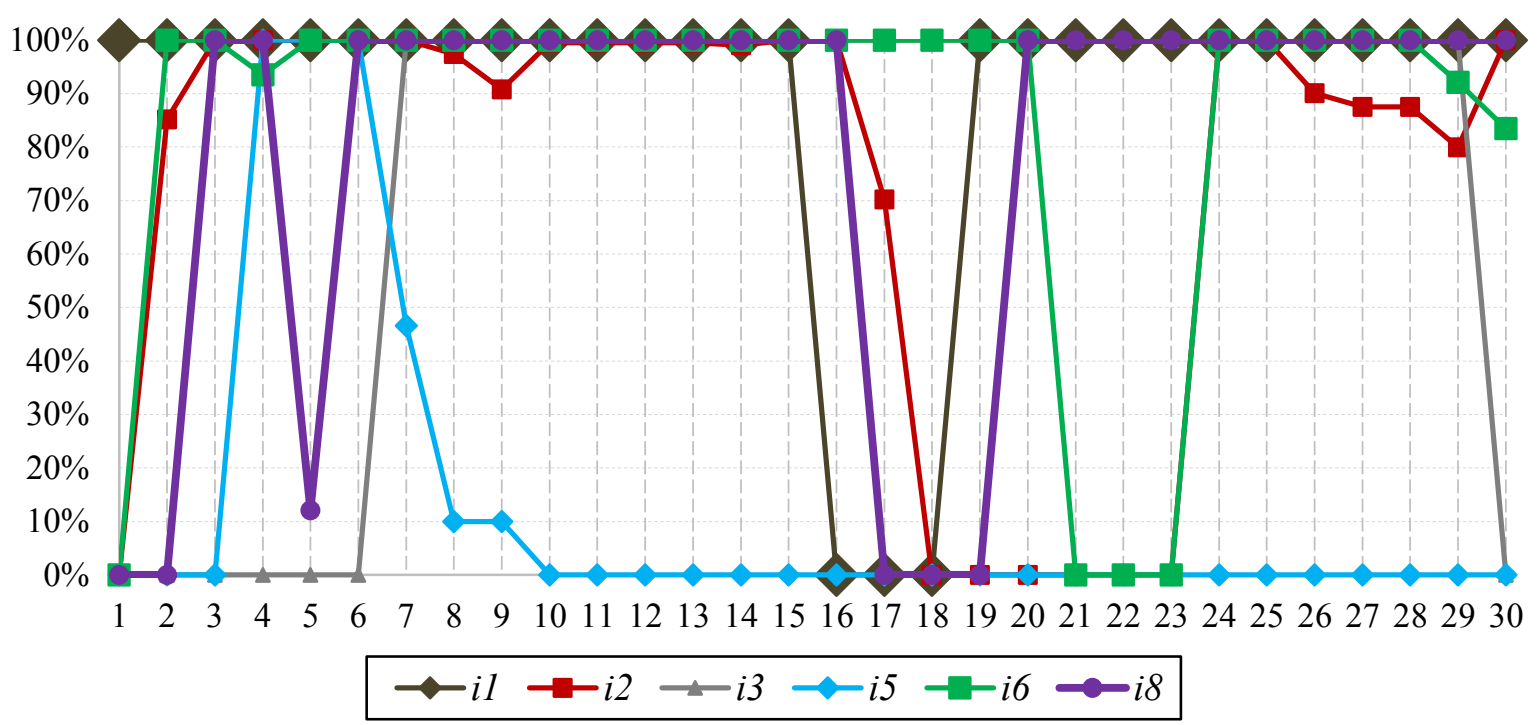

Figure 14. Case Study 2 - Integrated Approach: Normalized operating level profiles for utility and production units.

Figure 14 displays the normalized operating level profiles for utility and production units, having as a reference the maximum operating level of each unit as given in Table 1 . In the utility system, utility units $i 1$ to $i 3$ operate at their maximum operating levels throughout the planning horizon (excluding their cleaning periods). Utility unit $i 5$, which can generate only utility resource $e 2$, operates from day 4 to 9 to satisfy the needs for utility resource $e 2$. Maximum production level for utility units $i 5$ is observed from day 4 to 6 because utility unit $i 3$ is offline (refer to Figure 13). Then, the production level for utility unit i5 reduces to minimum because utility unit $i 3$ starts up in day 7 . In the production system, production units 
i6 and i8 operate in their maximum capacity almost in all time periods in order to satisfy the high demand for product resources.

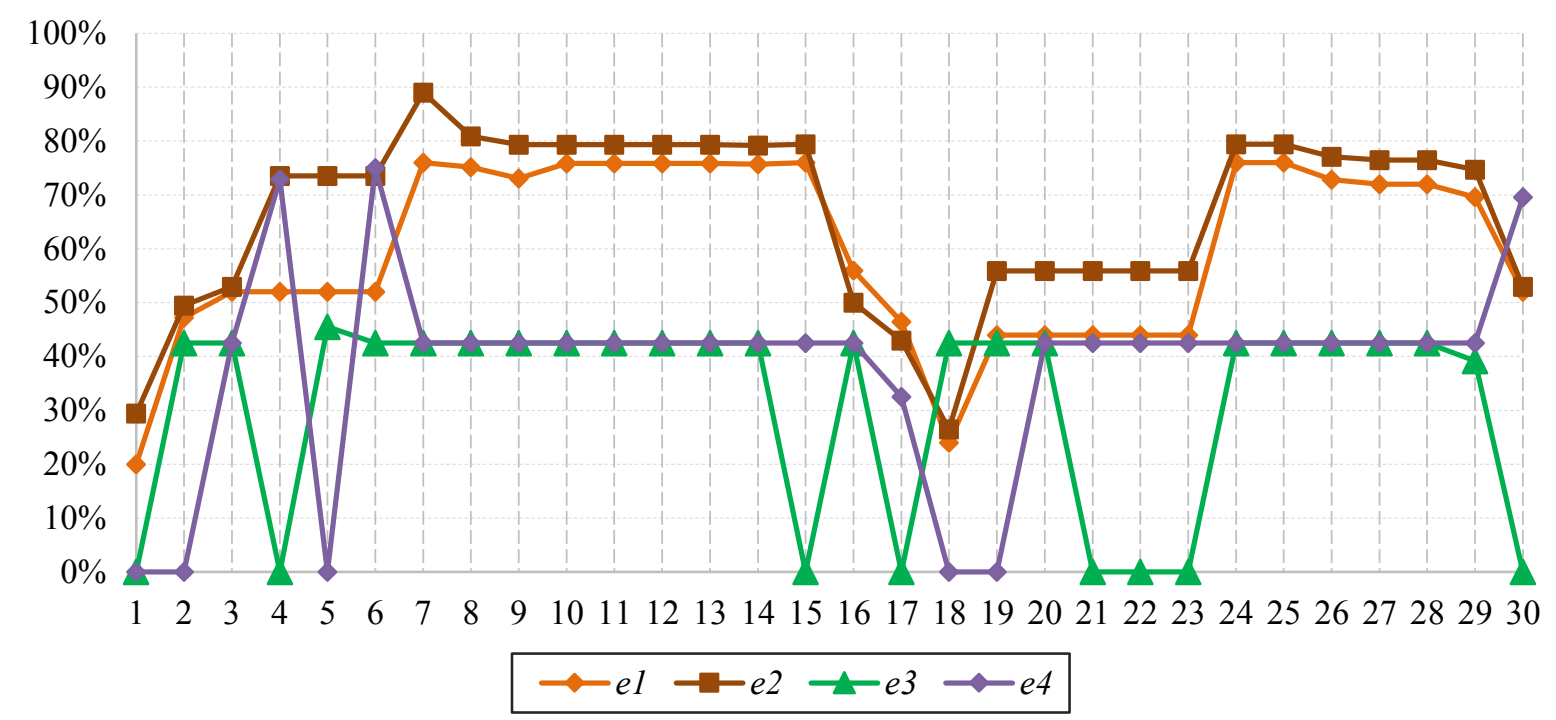

Figure 15. Case Study 2 - Integrated Approach. Normalized total production profiles for utility and final product resources.

Figure 15 displays the normalized total production profiles for every utility and final product resource. The total production for each resource is calculated by having the cumulative production of the resource from each unit divided by the maximum total resource capacity from all units. The production trends for utility resources $e 1$ and $e 2$ follow quite a similar trend throughout the planning horizon, mainly due to the presence of three utility units that cogenerate both utility resources. The only differences are observed when utility unit i5 operates from day 4 to 9 . There are higher production differences for utility resource $e 2$ than that of the production of utility resource $e 1$. The total production level for utility resources $e 1$ and $e 2$ are considerably reduced when cleaning takes place for utility units between days 16 and 23. The production profiles for product resources $e 3$ and $e 4$ from day 7 to 14 and from day 24 to 28 are on the same level because the upper operating level of utility unit i6 (produces product resource $e 3$ ) and utility unit i8 (produces product resource $e 4$ ) in all these days are the same (see Table 1). In addition, when there is no production of a product resource in certain 
time periods (e.g., days 1, 4, 15, 17, 21, 22, 23, 30 for product resource $e 3$ and days 1, 2, 5, 18, 19 for product resource e4 ) its corresponding demand is fully satisfied from its associated inventory tank.

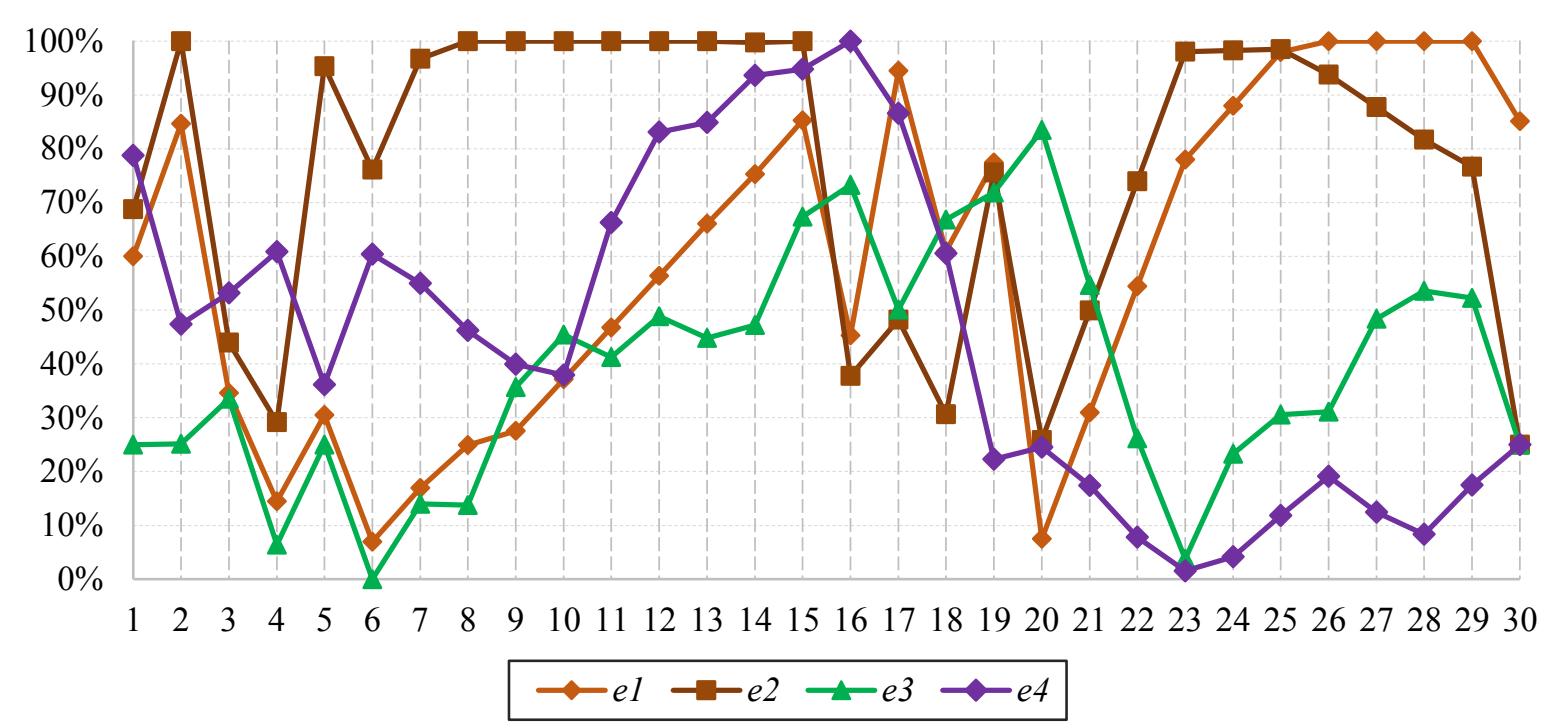

Figure 16. Case Study 2 - Integrated Approach. Normalized inventory profiles for utility and product resources.

Figure 16 displays the normalized inventory profiles for utility and product resources. The maximum inventory levels $\left(\xi_{(e, z)}^{\max }\right)$ are the reference values here. It is observed that, high inventory level for utility and product resources at the beginning of planning horizon because of initial inventory levels. There are reduced inventory levels for utility and product resources on day 16 to 23 because cleaning of utility unit $i 1$ and $i 2$ and production unit $i 6$ and $i 8$ take place on these days. At the end of day 30 , the inventory level for utility $e 2$ and product $e 3$ and $e 4$ are not approaching zero due to terminal constraints are set to be $25 \%$ of the initial inventory. However, this is not the case for utility $e 1$ because all utility units (i.e., $i 1, i 2$ and $i 3$ ) that cogenerate both utilities are operating at their maximum operating capacities (refer to Figure 14). It is not possible to operate these utility units in a lower capacity at the end of the planning horizon because the utility demand for $e 2$ must be fully satisfied in order to meet the 
demand for products. Thus, the optimal solution reports a $25 \%$ of inventory level for utility $e 2$ and a much higher inventory level for utility $e 1$ at the end of planning horizon.

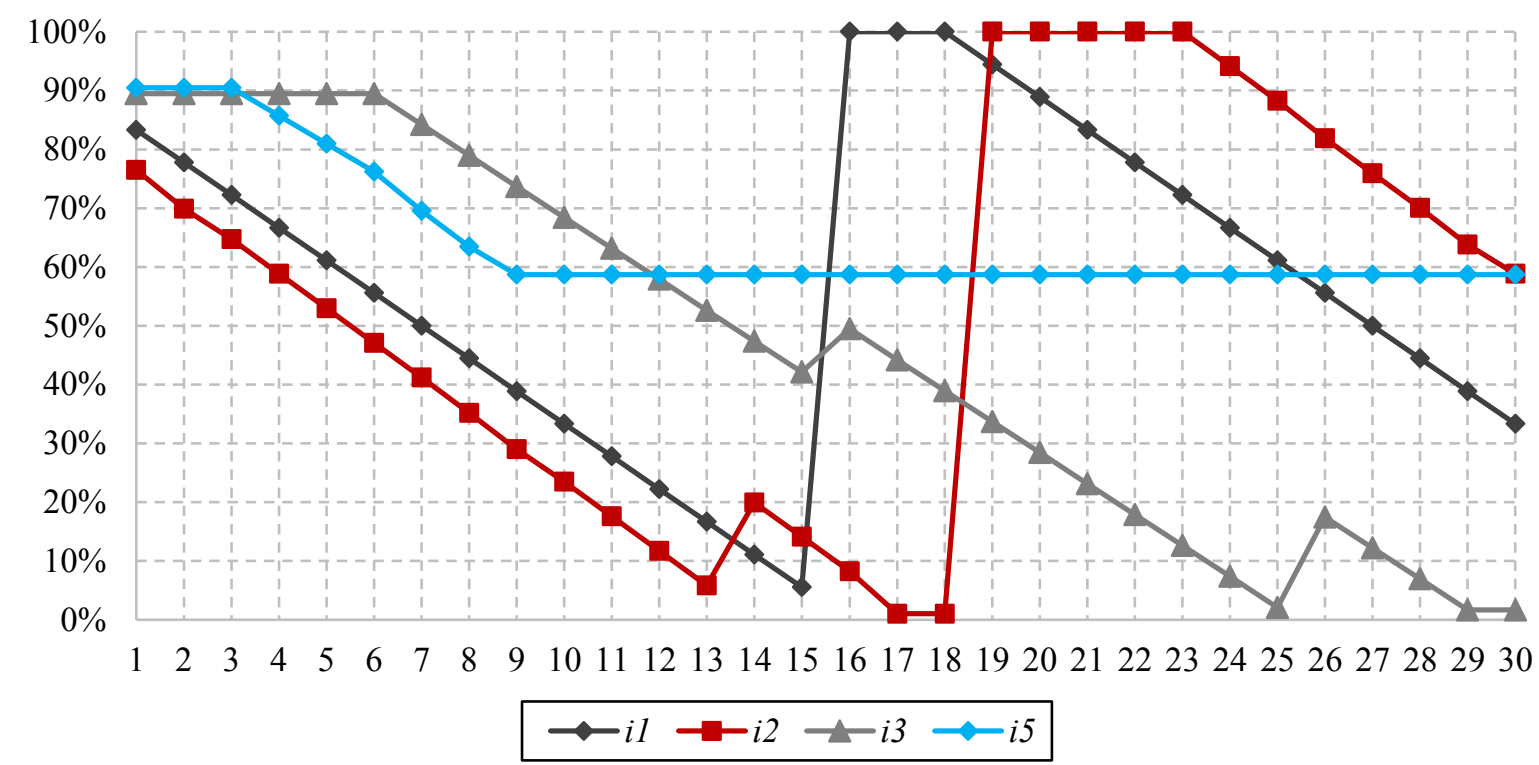

Figure 17. Case Study 2 - Integrated Approach: Performance level profiles for utility units per time period.

Figure 17 shows the performance level profiles for utility units that are subject to conditionbased cleaning. The performance level of a unit depends on its cumulative time of operation and its operating levels deviation. Here, it can be seen when the performance of utility units $i 1$ and $i 2$ is fully recovered once an offline cleaning occurs. It is also observed that utility unit i2 partially recovers its performance through an online cleaning in day 14 , and it continues operating until reaching its critical performance level in day 17 . The performance degradation of utility unit $i 5$ declines in a slightly varied rate (i.e., no straight line decline) from day 7 to 9 due to the deviation of its operating level from its maximum operating capacity (see Figure 14). Utility unit $i 5$ shuts down in day 10 and remains idle for the remaining planning horizon, thus no cleaning task is performed after its shutdown. The performance levels of all operating utility units at the end of the planning horizon remain above $25 \%$ (due to the terminal constraints imposed) except for utility unit $i 3$ that does not operate in day 30 and therefore 
terminal constraint was not applied (see Figure 13). In practice, one could start an offline cleaning task on this unit at the last period of the planning horizon to completely restore its performance.

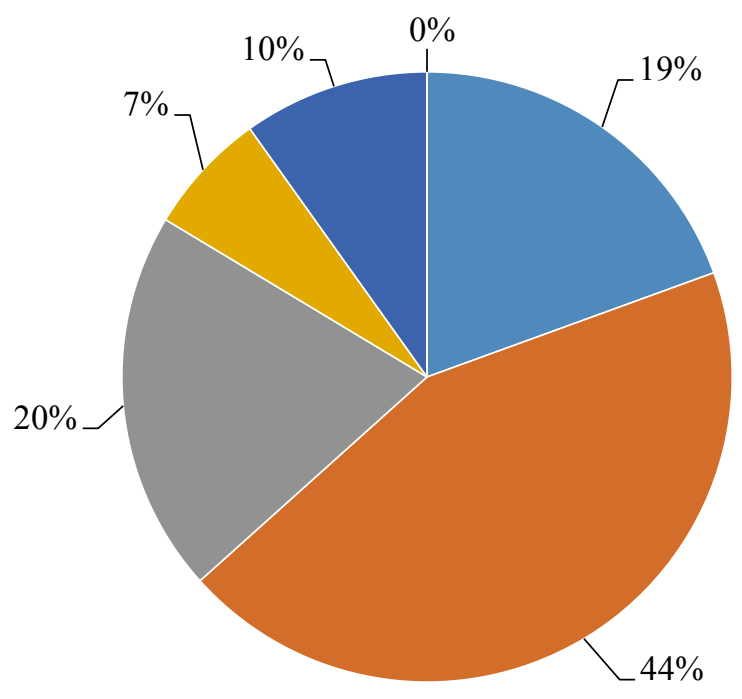

- Startup and shutdown cost

- Operating cost for utility units

- Operating cost for production units

Cleaning cost for units

- Extra power consumption cost

- Purchase cost for resources

\section{Figure 18. Case Study 2 - Integrated Approach: Total cost breakdown (percentage).}

Figure 18 demonstrates the total cost breakdown for the utility and production systems. As in the previous case study, the operating cost of the utility system remains the highest cost term. This is because the production levels of utility resources to satisfy the utility demand of the production system are much higher that the production levels of the production system. Also, variable and fixed utility costs are relatively expensive. The startup and shutdown cost and the operating cost of the production system are at $19 \%$ and $20 \%$ of the total cost, respectively. The extra energy consumption and cleaning costs are around $10 \%$ and $7 \%$.

\subsubsection{Results of Case Study 2 - Sequential Approach}

The same case study has been solved following the traditional sequential approach in order to make a comparison of its solution with the solution obtained by the proposed integrated approach. Figure 19 displays the optimal operational and cleaning plan for the sequential approach. In comparison with the integrated approach, a higher number of online cleaning tasks for utility units is observed. Some major observations are that: (i) utility unit i4 still remains 
inactive throughout the whole planning horizon; (ii) utility unit is operates in a larger number of time periods than before; and (iii) production unit $i 7$ now operates in most of the time periods and production unit i8 operates less time in the 30-day planning horizon.

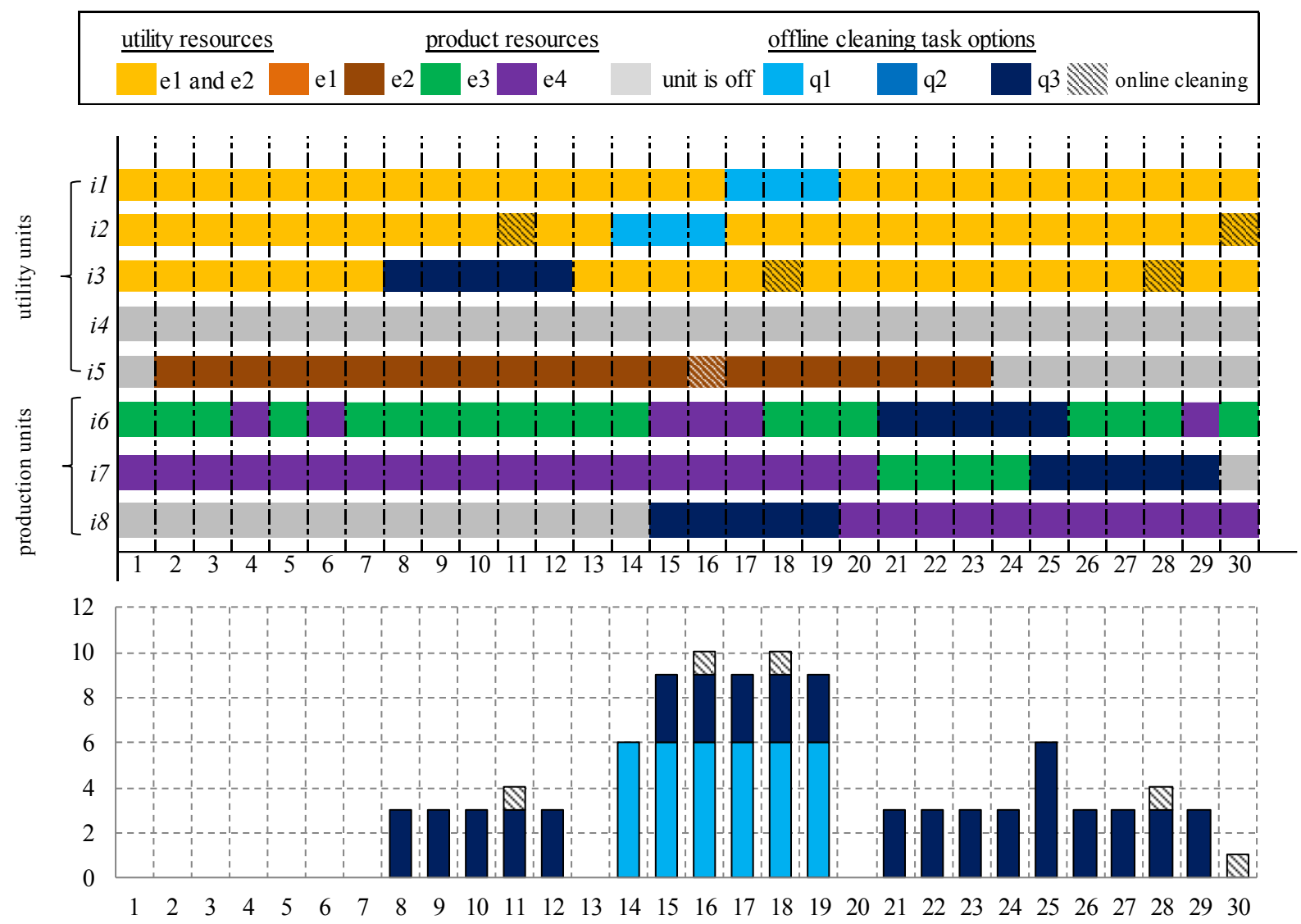

Figure 19. Case Study 2 - Sequential Approach: Operational and cleaning plan for production and utility systems and total utilization profile of cleaning resources. 


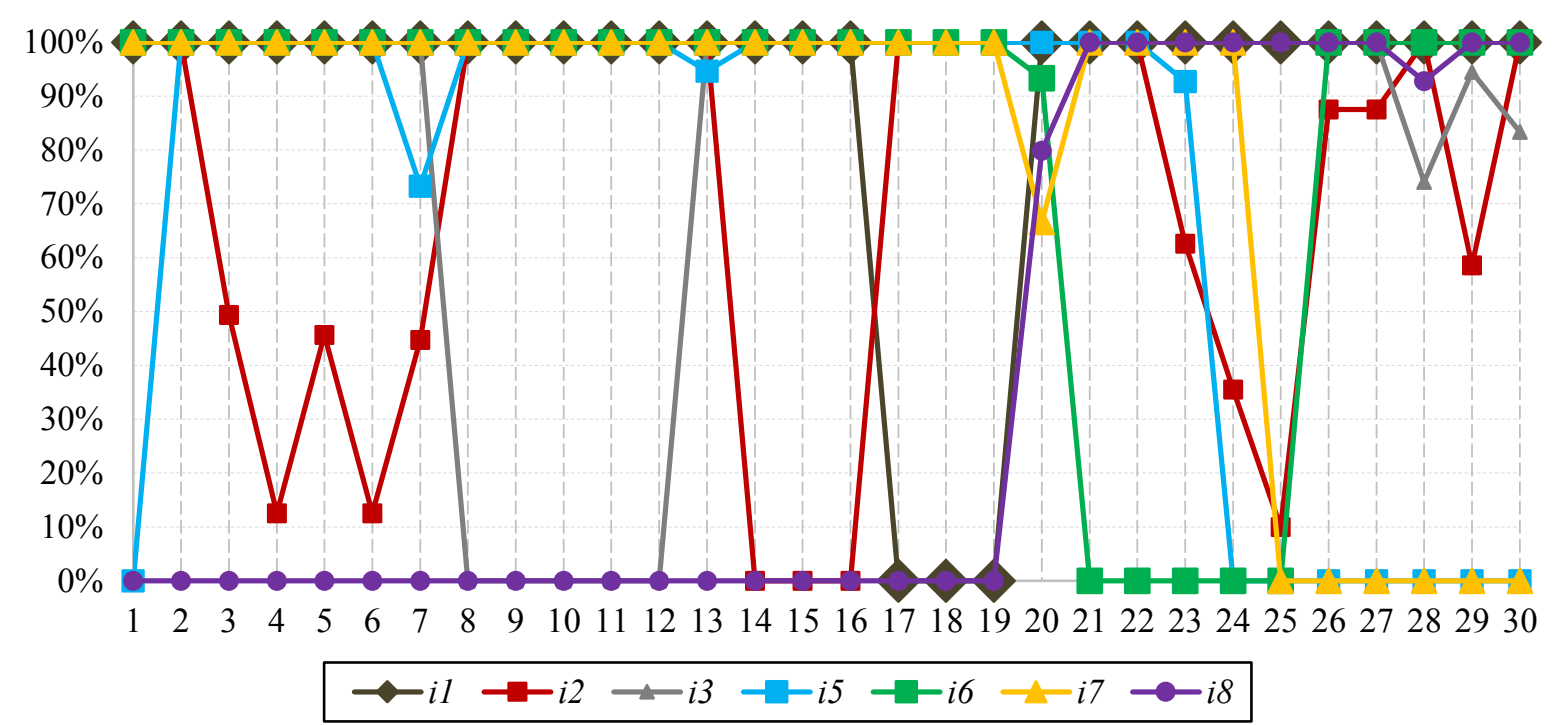

Figure 20. Case Study 2 - Sequential Approach: Normalized operating level profiles for utility and production units.

Figure 20 shows the normalized operating level profiles for utility and production units of the solution of the sequential approach. In comparison with the solution of the integrated approach (Figure 14), utility units $i 1$ and $i 3$ operate at their maximum operating levels while the operating level of utility unit $i 2$ varies in order to accommodate the demand for utility resources. Utilized production units operate on their maximum operating capacities most of the times. 


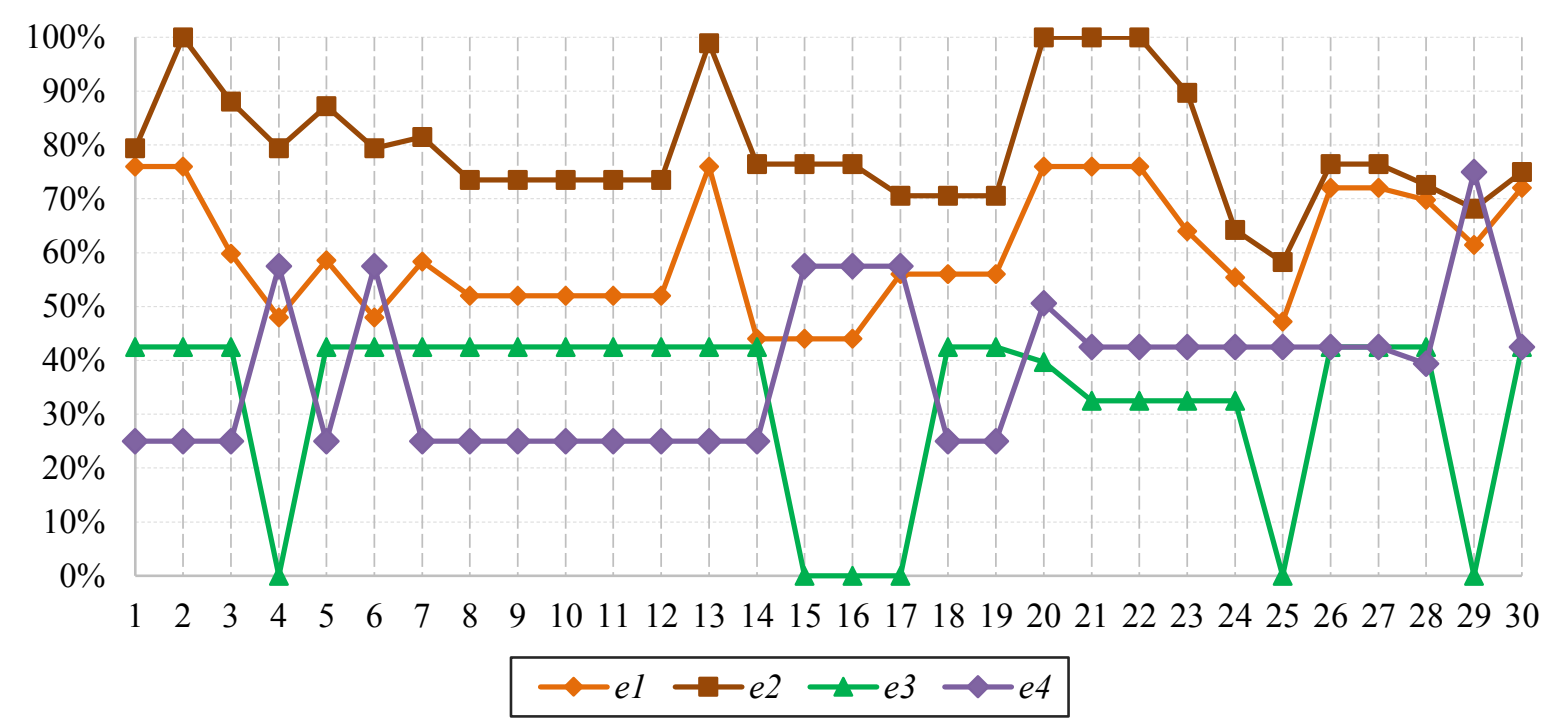

Figure 21. Case Study 2 - Sequential Approach. Normalized total production profiles

\section{for utility and product resources.}

Figure 21 displays the normalized total production profiles for utility and product resources. The production profiles for utility resources $e 1$ and $e 2$ follow quite a similar pattern throughout planning horizon. Since a production unit can produce at most one product resource per time period and there is a limited number of production units, the production profile for product resource $e 3$ follows the opposite trend of that of product resource $e 4$. 


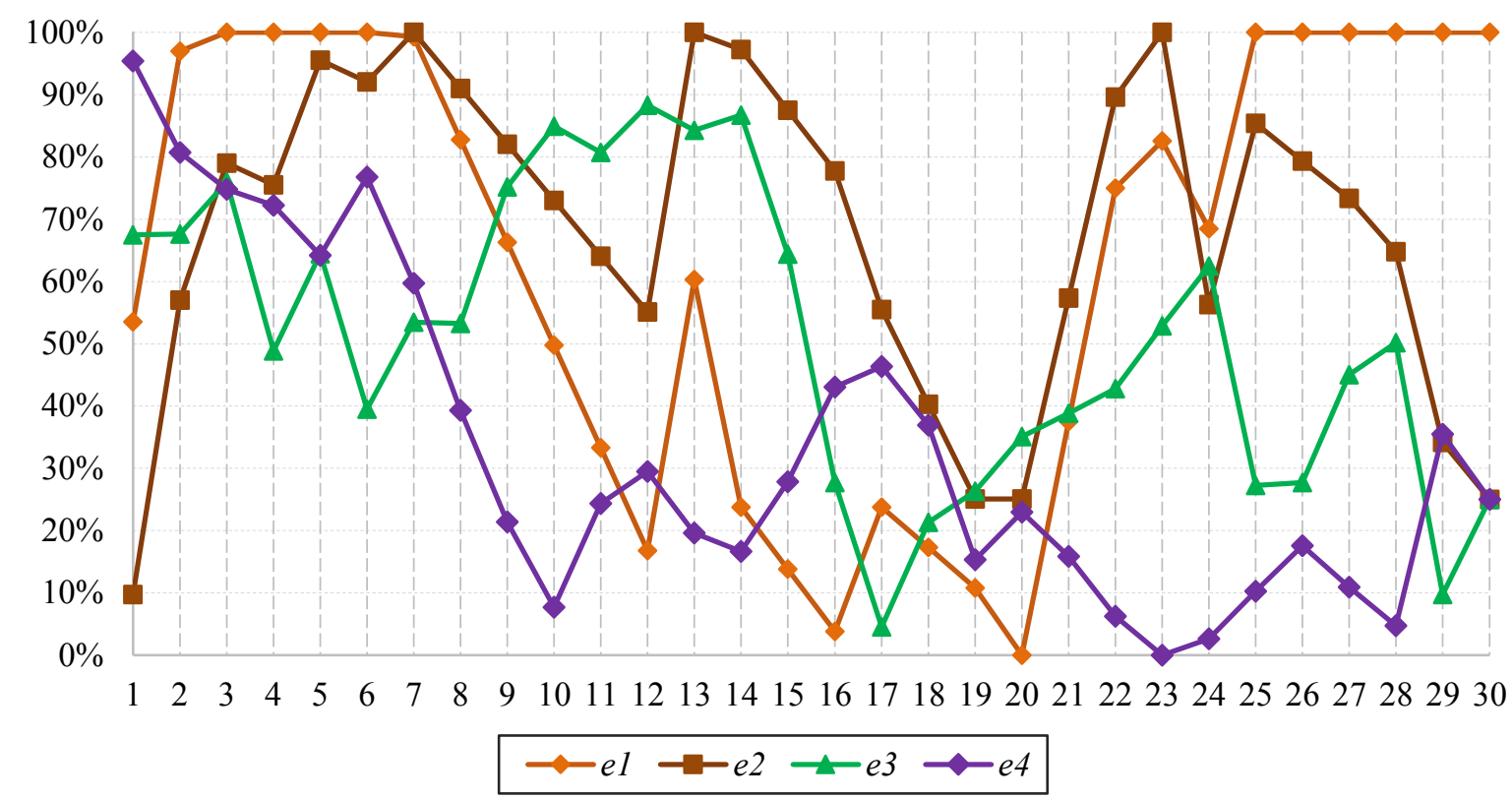

Figure 22. Case Study 2 - Sequential Approach: Normalized inventory profiles for utility and product resources.

The normalized inventory profiles for utility and product resources are shown in Figure 22. The inventory levels for utility resources $e 1$ and $e 2$ are lower in day 14 to 19 , which is due to the offline and online cleaning of the utility units (see Figure 19). The inventory level for product resource $e 3$ reduces considerably from day 15 and 17 because no production unit is producing product resource $e 3$ in these days and the corresponding demand is satisfied exclusively from its inventory tank. At the end of day 30, the inventory level for utility resource $e 2$ and product resources $e 3$ and $e 4$ are equal to $25 \%$ of their maximum inventory capacity due to the terminal constraints imposed. However, a much higher inventory level is for utility resource $e 1$ is reported, similarly to the solution of the integrate approach. As explained before, this is mainly do the existence of utility cogeneration units that cogenerate both utilities under different generation ratios (see Table 3). 


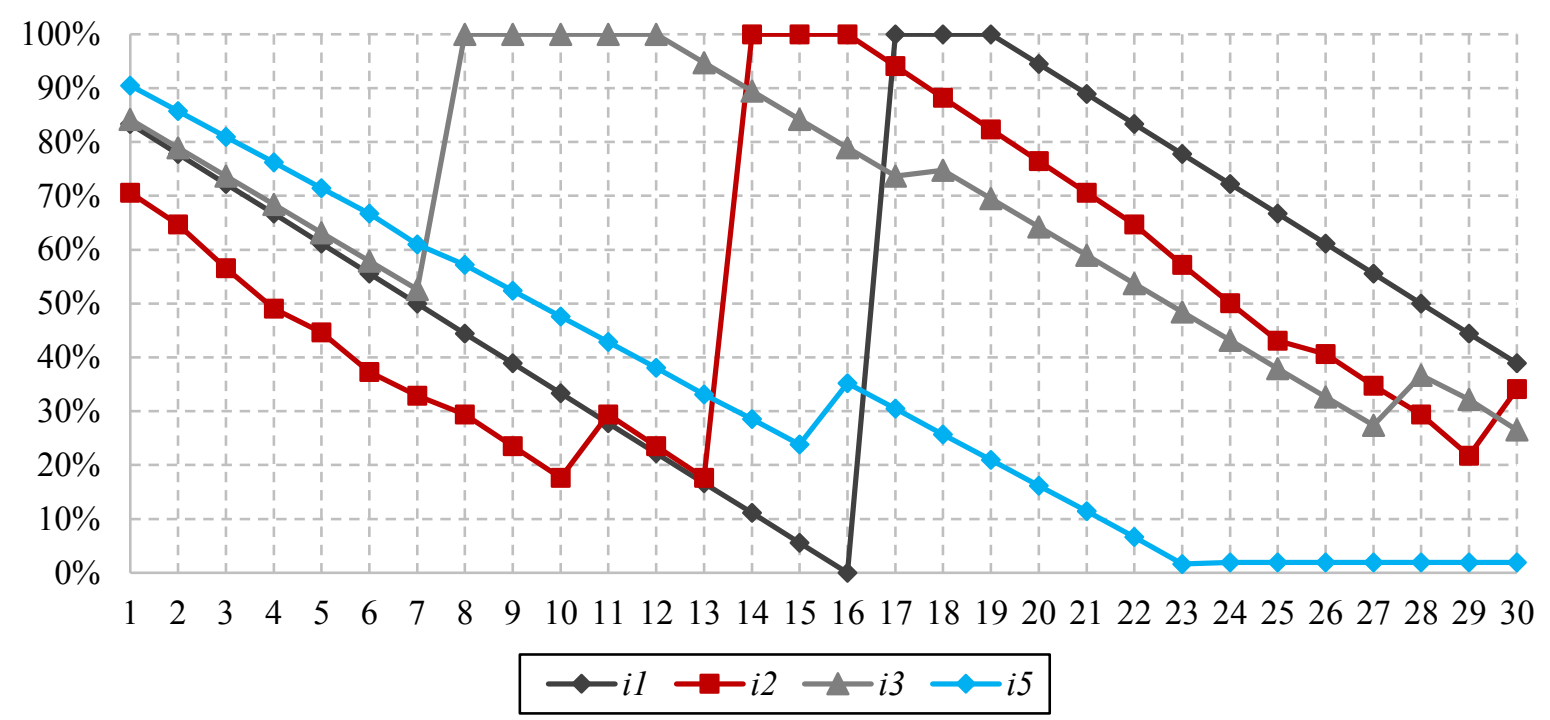

Figure 23. Case Study 2 - Sequential Approach. Performance level profiles for utility units per time period.

The performance level profiles for active utility units are displayed in Figure 23. It can be seen that the performance level of utility unit i2 decreases according to the variation in its operating levels. Utility units $i 1, i 2$ and $i 3$ fully recover their performances by undergoing offline cleaning tasks, while utility unit i5 undergoes online cleaning in day 16 to partially recover its performance. The performance levels of all operating utility units at the end of the planning horizon remain above $25 \%$ (due to the terminal constraints imposed) except for utility unit i5 that does not operate in day 30 and therefore terminal constraint was not applied (see Figure 13). In practice, one could perform an offline cleaning on this unit after day 22 to completely restore its performance by the end of the planning horizon. 


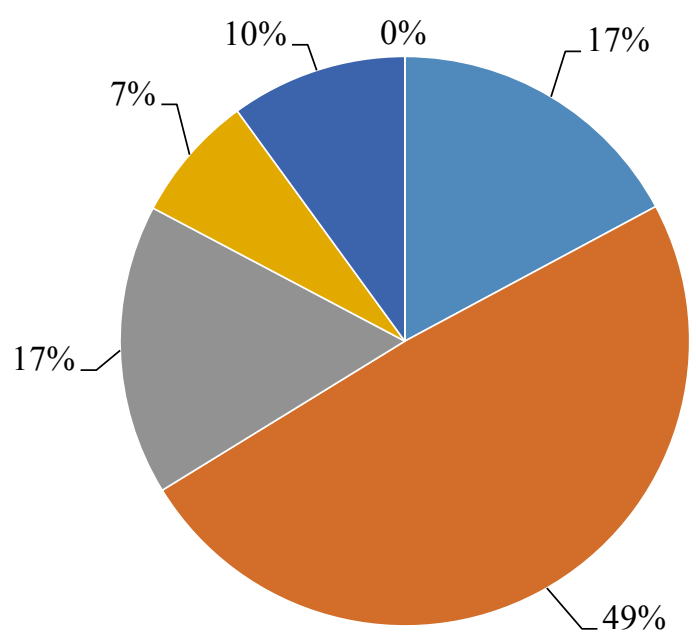

$$
\begin{aligned}
& \text { Startup and shutdown cost } \\
& \text { Operating cost for utility units } \\
& \text { Operating cost for production units } \\
& \text { Cleaning cost for units } \\
& - \text { Extra power consumption cost } \\
& \square \text { Purchase cost for resources }
\end{aligned}
$$

\section{Figure 24. Case Study 2 - Sequential Approach: Total cost breakdown (percentage).}

Figure 24 shows the total cost breakdown for the solution of the sequential approach. The operating cost for utility units is $49 \%$ which is $5 \%$ higher than the percentage of the operating cost of the integrated approach (refer to Figure 10). This is because utility unit i5 operates for a longer horizon in sequential approach in comparison with the integrated approach.

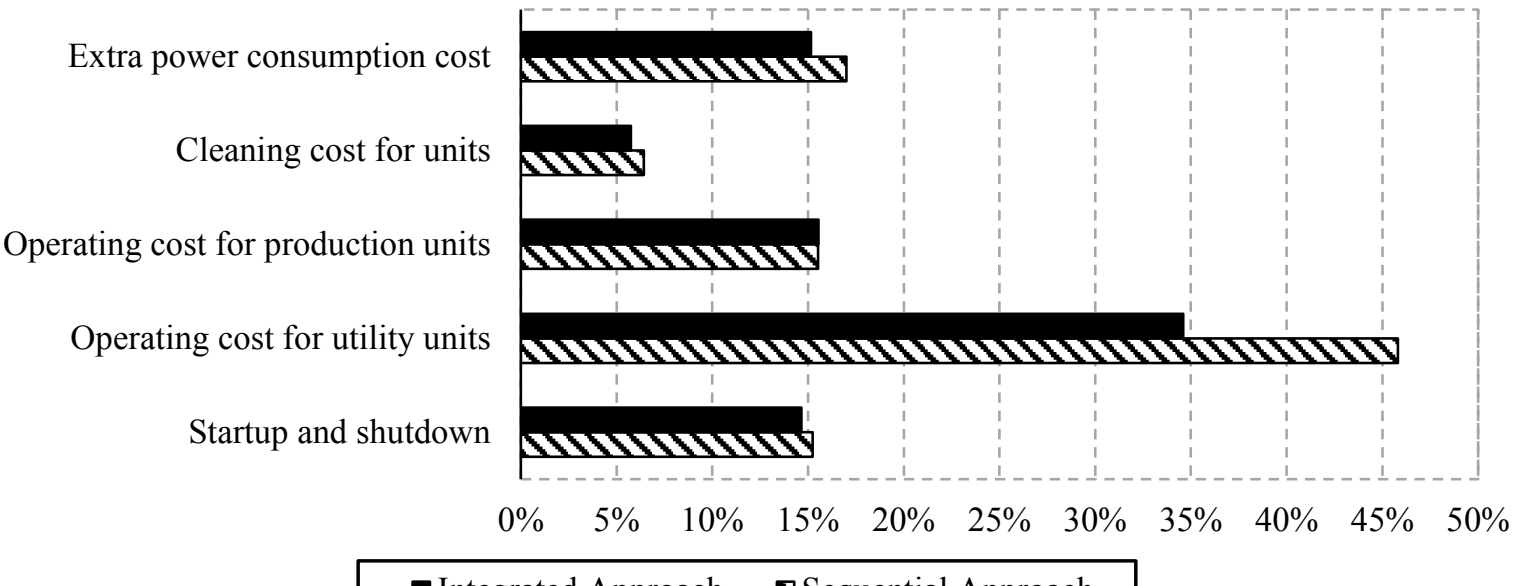

Figure 25. Case Study 2: Cost term comparison of integrated and sequential approach.

Figure 25 shows the cost comparison of the solutions derived by following the integrated and the sequential approach. Each cost term for both solutions is divided by the total cost for sequential approach (which is higher than that of the integrated approach). The major cost difference between the solution of the integrated and the sequential approach is the operating 
cost for utility units that is about $13 \%$. This difference in the operating cost for utility system affect strongly the total cost of the solution found by the sequential approach. The extra energy consumption cost, cleaning cost and startup and shutdown cost show cost differences of around $1 \%$. The operating cost for production units is almost the same for both approaches.

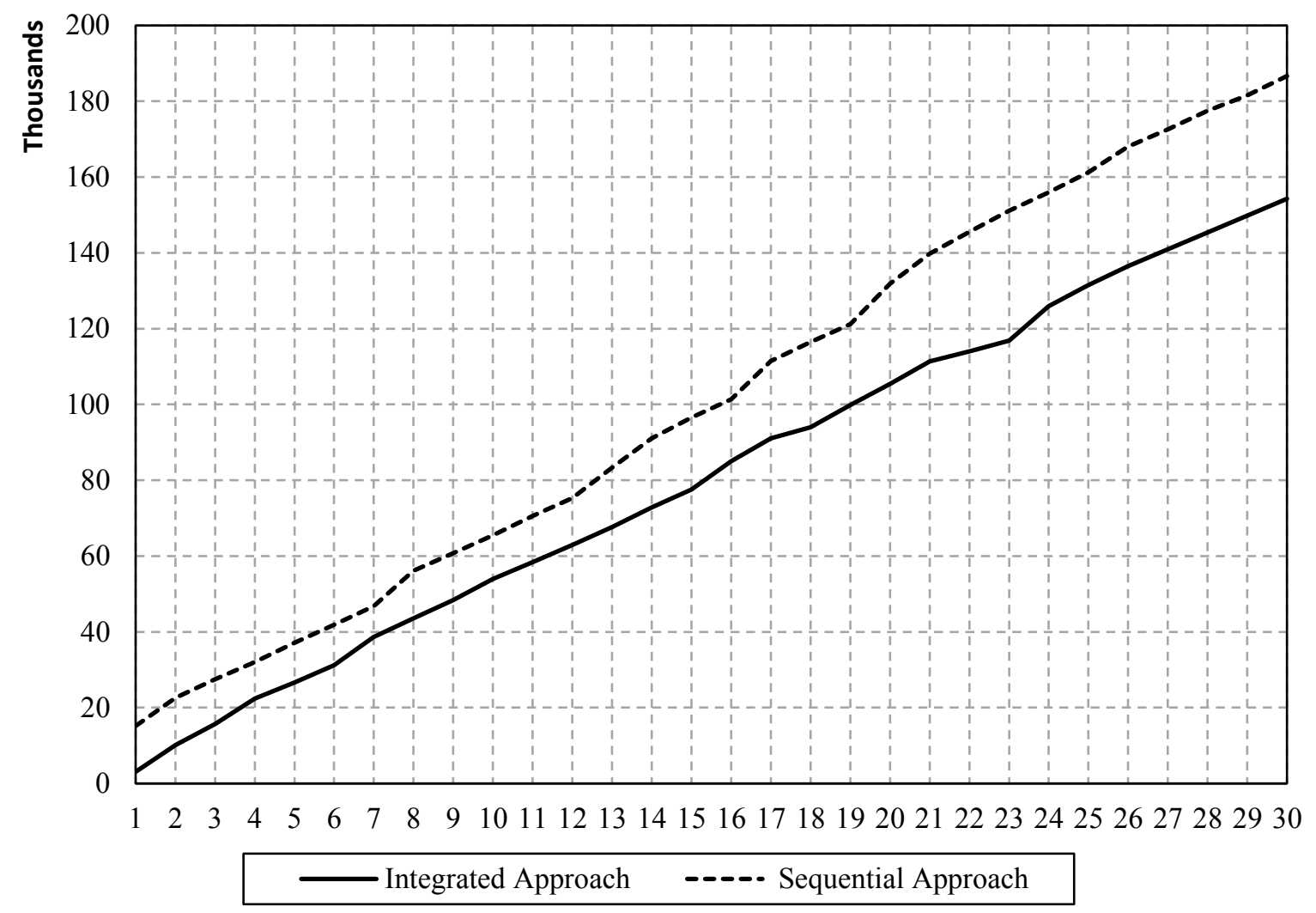

Figure 26. Case Study 2: Aggregated total cost for integrated and sequential approach.

Figure 26 displays the evolution of the total cost value over time for both approaches. This difference significantly increases by the end of the planning horizon. The vertical difference between the two lines in the graph shows the difference of the total cost between the two solutions. In particular, it is observed that the total cost of the solution of the integrated approach is $17 \%$ lower than that of the sequential approach demonstrating clearly the benefits of the proposed integrated approach.

\subsection{Case Study 3: Integrated Planning of Utility and Production Systems via Rolling Horizon Approach.}


In this example, the reactive integrated planning problem of utility and production systems through a rolling horizon approach is considered in order to show how the proposed optimization framework can be readily used in a dynamic environment. For the rolling horizon approach, a prediction horizon equal to 15 time periods and a single-period control horizon have been used. A time period is equal to one day. The total planning horizon of interest is 30 days, therefore a total number of 30 iterations have been solved (30 optimization problems). For each iteration, a planning problem for the next 15 time periods is solved with updated information of the current state of the overall system and the demand for product resources. Only the solution of the first time period of the current prediction horizon is applied at each iteration, and the initial state of the next iteration is updated accordingly. In this case study, all utility and production units are subject to alternative condition-based cleaning policies.

\subsubsection{Description of Case Study 3}

This example is a slight modified version of the previous case study. The main parameters (Table 1-4) and operational costs (Table 5) are as before, and the demands for products in the first 30 days is the same as in Case Study 2. In order to apply the rolling horizon approach, they have been considered demands for products for 14 additional time periods (i.e., until day 44) which follow similar a distribution as in the previous periods. Minimum runtime and shutdown times are the same as in the previous examples. Here, all utility and production units are subject to condition-based cleaning, for which there are three alternative cleaning tasks options as before. There is a limited number of available cleaning resources equal to 12 units of cleaning resources.

The parameters that refer to condition-based offline and online cleaning are defined in Table 8 are: (i) extra energy consumption limit ( $\left.v_{i}^{\max }\right)$; (ii) cumulative time degradation rate $\left(\delta_{i}\right)$;(iii) operating level degradation rate $\left(\delta_{i}^{q}\right)$; (iv) minimum time between two consecutive online cleaning tasks $\left(\gamma_{i}^{o n}\right)$; (v) recovery factor of the online cleaning $\left(\rho_{i}^{\text {rec }}\right)$; (vii) reduction factor of 
the operating level for online cleaning $\left(q_{i}^{o n}\right)$; and (vi) resource requirement for online cleaning of a unit $\left(\vartheta_{i}^{o n}\right)$. In addition, the parameters that define the initial state for this case study are given in Table 9. Terminal constraints for each prediction horizon are the same as in the previous case study.

Table 8. Case Study 3: Parameters related to the condition-based cleaning of utility and production units.

\begin{tabular}{lllllllll}
\hline Parameter & $i 1$ & $i 2$ & $i 3$ & $i 4$ & $i 5$ & $i 6$ & $i 7$ & $i 8$ \\
\hline$v_{i}^{\text {max }}$ & 162 & 153 & 247 & 200 & 210 & 240 & 242 & 247 \\
$\delta_{i}$ & 9 & 9 & 13 & 10 & 10 & 12 & 11 & 13 \\
$\delta_{i}^{q}$ & 6.75 & 6.75 & 9.75 & 7.50 & 7.50 & 9 & 8.25 & 9.75 \\
$\gamma_{i}^{\text {on }}$ & 10 & 10 & 10 & 10 & 10 & 10 & 10 & 10 \\
$\rho_{i}^{\text {rec }}$ & 0.20 & 0.20 & 0.20 & 0.20 & 0.20 & 0.20 & 0.20 & 0.20 \\
$q_{i}^{\text {on }}$ & 0.05 & 0.05 & 0.05 & 0.05 & 0.05 & 0.10 & 0.10 & 0.10 \\
$\vartheta_{i}^{\text {on }}$ & 1 & 1 & 1 & 1 & 1 & 1 & 1 & 1 \\
\hline
\end{tabular}

Table 9. Case Study 3: Initial state of utility and production units.

\begin{tabular}{lcccccccc}
\hline Parameter & $i 1$ & $i 2$ & $i 3$ & $i 4$ & $i 5$ & $i 6$ & $i 7$ & $i 8$ \\
\hline$\tilde{\rho}_{i}$ & 9 & 16 & 17 & 4 & 18 & 8 & 5 & 17 \\
$\tilde{\gamma}_{i}^{o n}$ & 22 & 10 & 25 & 41 & 43 & 14 & 39 & 6 \\
$\tilde{\omega}_{i}$ & 9 & 6 & 17 & 0 & 0 & 8 & 0 & 22 \\
$\tilde{\psi}_{i}$ & 0 & 0 & 0 & 28 & 9 & 0 & 29 & 0 \\
$\tilde{\rho}_{i}^{d q}$ & 0 & 0 & 0 & 0 & 0 & 0 & 0 & 0 \\
\hline
\end{tabular}




$\begin{array}{lccc}\tilde{\beta}_{(e l, z 1)} & 60 & \text { units } & \text { Initial inventory for utility resource } e 1 \\ \tilde{\beta}_{(e 2, z 2)} & 93 & \text { units } & \text { Initial inventory for utility resource } e 2 \\ \tilde{\beta}_{(e 3, z 3)} & 132 & \text { units } & \text { Initial inventory for product resource } e 3 \\ \tilde{\beta}_{(e 4,74)} & 56 & \text { units } & \text { Initial inventory for product resource } e 4\end{array}$

\subsubsection{Results of Case Study 3 - Integrated Approach}




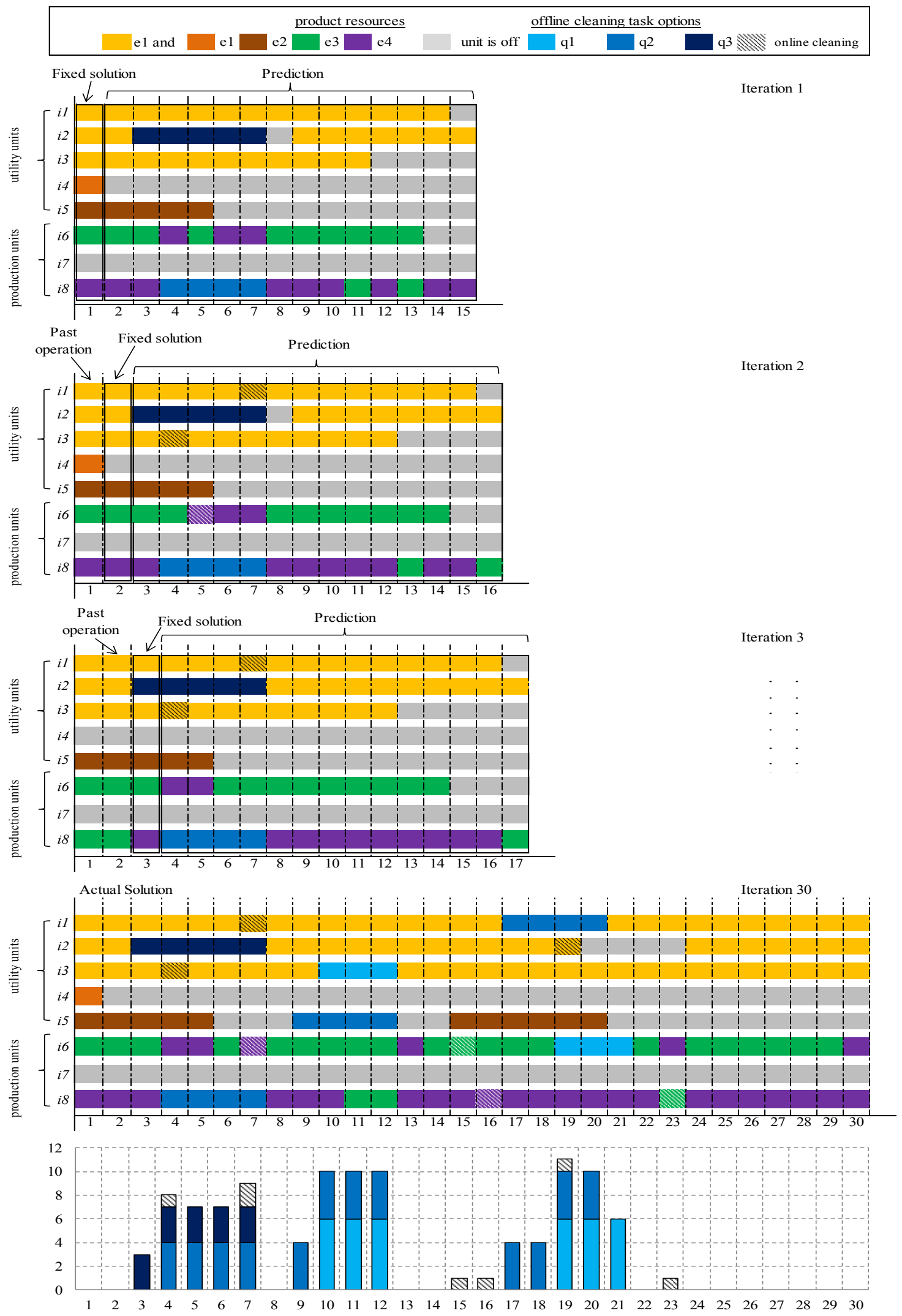

Figure 27. Case Study 3 - Rolling Horizon Integrated Approach: Plan generation via rolling horizon and total utilization profile of cleaning resources. 
Figure 27 displays how the final plan for the 30-day horizon is constructed through the solution obtained from each iteration (an example of the first three iterations is included). The last Gantt chart in this figure gives the implemented operational and cleaning plan and the total utilization profile of cleaning resources for the planning horizon considered. For the first iteration, the planning problem is solved for time periods 1 to 15 . Only the solution of the first time period is saved. In the second iteration, a new optimization problem for time periods 2 to 16 is solved having as initial state of the system the past solution for the first time period of the previous iteration. And, the rolling horizon method continues until all 30 iterations are solved (see also Figure 3)

Six offline and seven online cleaning tasks for utility and production units are observed in the implemented Gantt chart. There are some simultaneous condition-based offline cleaning tasks for some units, as listed below: (i) utility unit $i 2$ and production unit i8 from day 4 and 7; (ii) utility units $i 5$ and $i 3$ from days 10 and 12; and (iii) utility unit $i 1$ and production unit $i 6$ in days 19 and 21. In addition, simultaneous online cleanings is observed for utility unit $i 1$ and production unit $i 6$ in day 7.

Utility unit $i 4$, which can only produce utility resource $e 1$, operates just in day 1 because utility resource $e 1$ has enough supply from the utility units that can cogenerate both utility resources. Utility unit $i 5$, which can produce utility resource $e 2$, operates for two shortduration period, from day 1 to 5 and from day 15 to 20 , because utility units $i 2$ and $i 1$ are closed for offline cleaning in some of these days. It is also observed that production unit $i 7$ remains idle for the whole planning horizon, because the demand for product resources is fully satisfied by the other production units. 


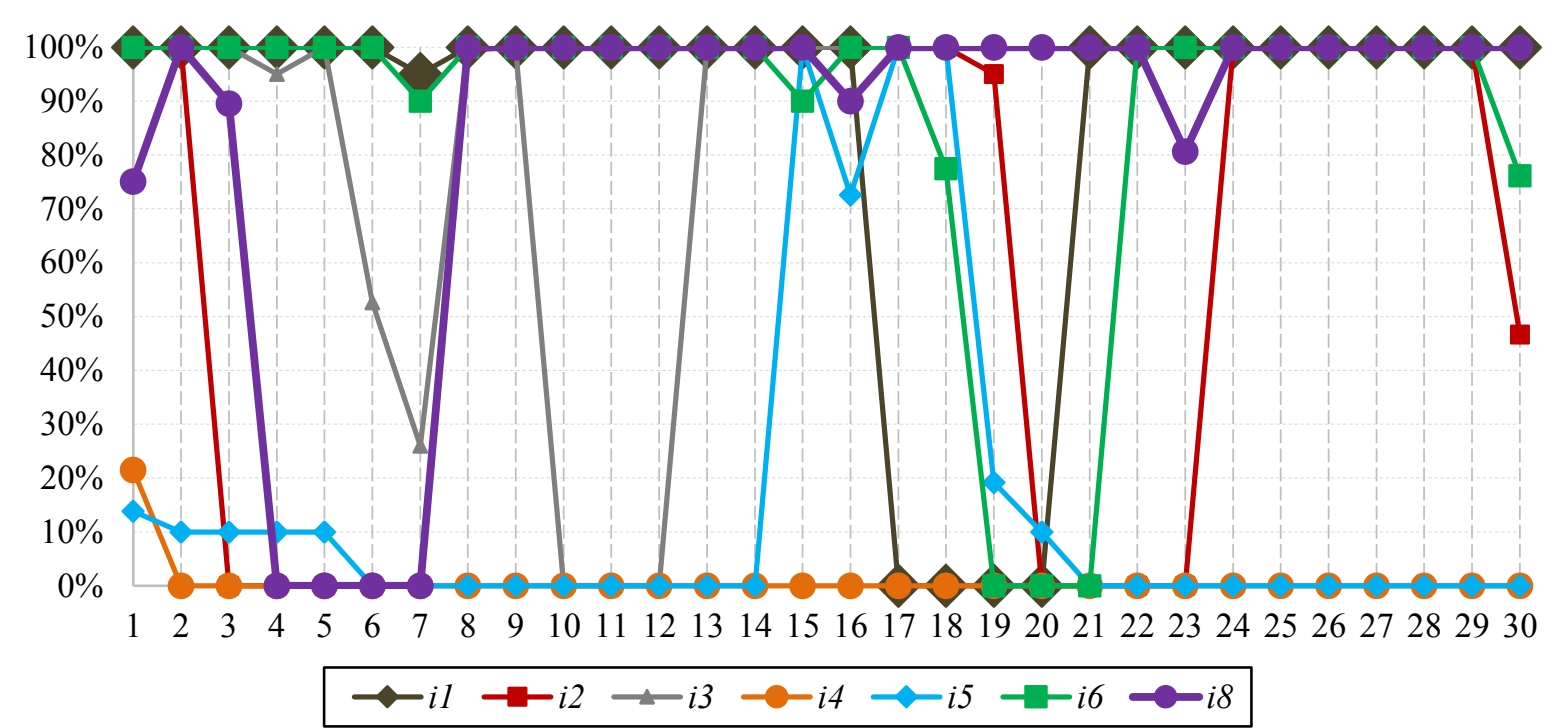

Figure 28. Case Study 3 - Rolling Horizon Integrated Approach: Normalized operating level profiles for utility and production units.

The normalized operating level profiles for all units are displayed in Figure 28. In the utility system, utility units $i 1$ to $i 3$ operate at their maximum operating levels throughout the planning horizon (excluding their cleaning periods). Utility unit $i 5$, which can generate only utility resource $e 2$, operates in a shorter operating range to satisfy the varied needs for utility resource $e 2$. In the production system, production units $i 6$ and $i 8$ operate at their maximum operating levels almost in all time periods to satisfy the high demand for product resources. 


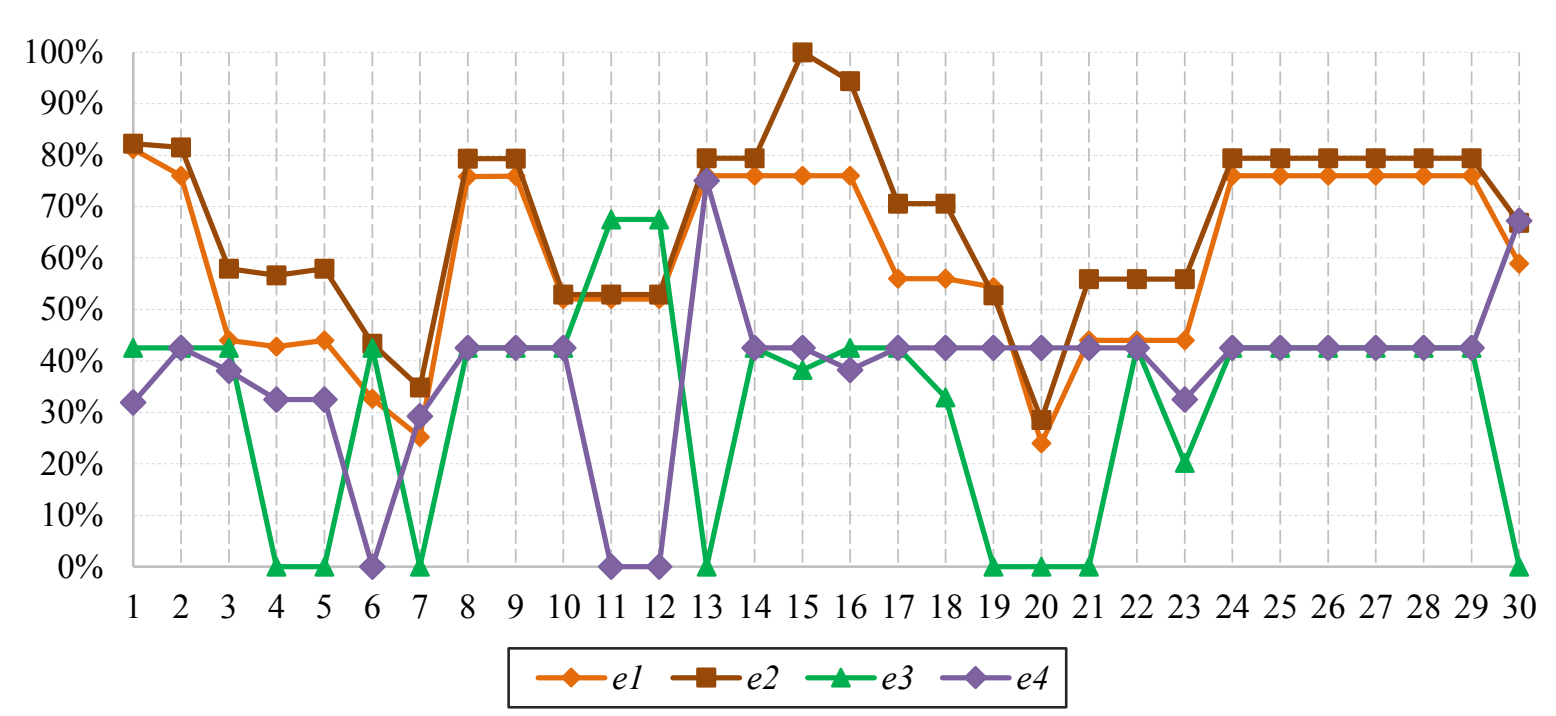

Figure 29. Case Study 3 - Rolling Horizon Integrated Approach: Normalized total production profiles for utility and product resources.

Figure 29 depicts the normalized total production profiles for each utility and product resource. The production of each resource is calculated by having the cumulative production of the resource from each unit divided by the maximum total resource production capacity of all units. Similar production trends are observed for utility resources el and $e 2$ mainly due to the presence of three utility units that cogenerate both utility resources. The only differences are observed when utility unit $i 5$ operates from day 1 to 5 and from day 15 to 20 . There are higher production differences of utility resource $e 2$ in comparison to utility resource $e 1$. Meanwhile, the production levels for product resources $e 3$ and $e 4$ from day 8 to 10 and from day 24 to 29 are exactly the same because the upper operating level of utility unit i6 that produces product resource $e 3$ and the upper operating level of production unit is that are produces product resource $e 4$ in these days are the same (refer to Table 1). In addition, when there is no production of product resources in some time periods (e.g., days 4, 5, 7, 13, 19, 20, 21 for product resource $e 3$ and days $6,11,12$ for product resource $e 4$ ), the demands for product resources are fully satisfied through the inventory tanks for product resources. 


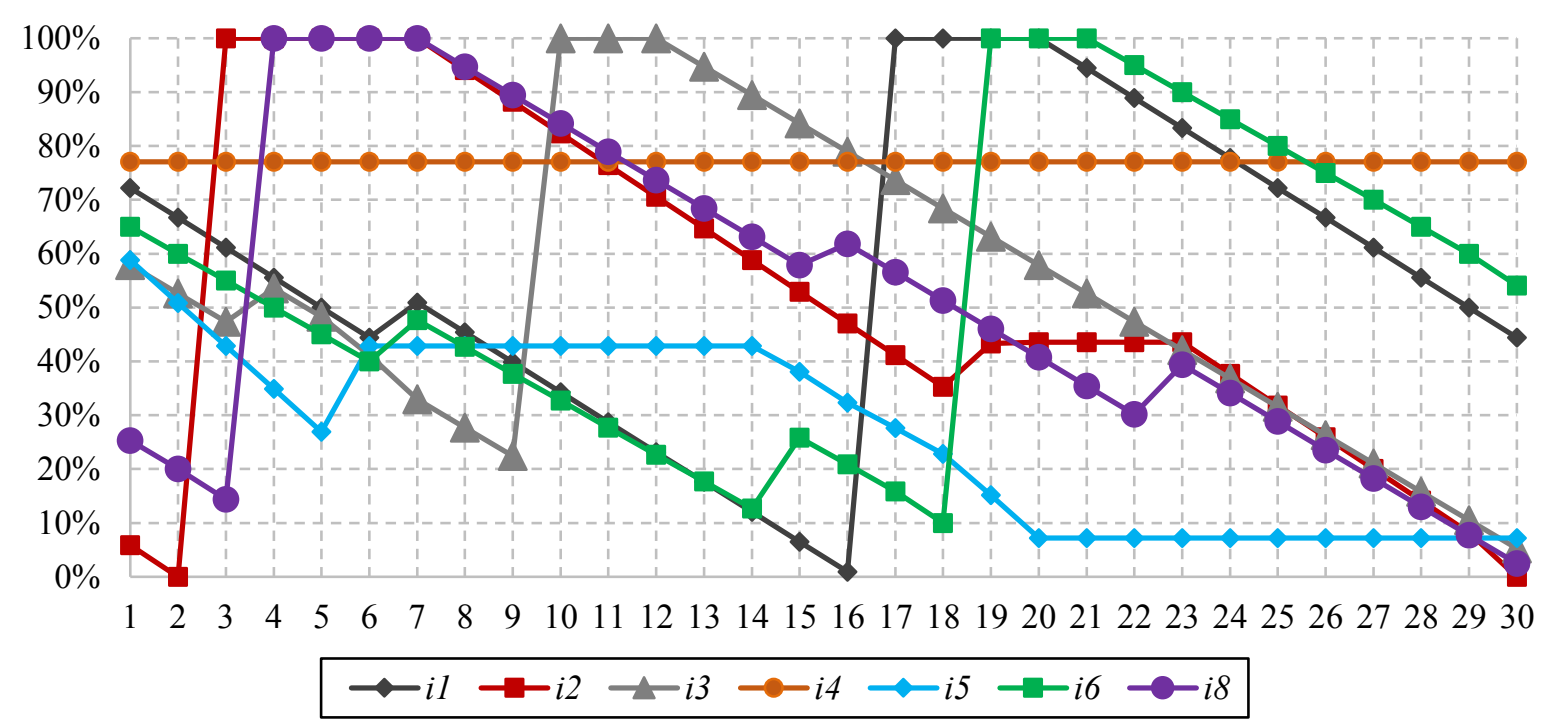

Figure 30. Case Study 3 - Rolling Horizon Integrated Approach: Performance level profiles for utility and production units per time period.

The performance level profiles for utility and production units are displayed in Figure 30. It is observed that utility unit $i 1$ undergoes online cleaning in day 7 to partially recover its performance and it continues operating until reaching its critical performance level in day 16 . The next day, utility unit $i 1$ is closed for offline cleaning in order to completely restore its full performance (i.e., clean condition). Production unit i6 undergoes two online cleanings (in day 7 and 15) and an offline cleaning in day 19. Utility unit i5 shows increased performance degradation from day 14 to 20 due to variation from its reference operating level (refer to Figure 28). It is also observed that utility unit $i 5$ reaches a very low performance level and eventually shuts down in day 21. No cleaning task takes place in this unit because it remains idle for the remaining planning horizon. In Figure 31, the performance levels of some operating units in day 30 are below $25 \%$ (i.e., terminal constraint) but this is not a violation of the corresponding terminal constraints. The solution of day 30 (including performance level values) has been derived from iteration 30 by solving a planning problem from time period 30 to time period 44, satisfying the terminal constraints for time period 44 . In other words, in iteration 30 , the 
terminal constraints apply for the last time period of the planning problem solved (i.e., day 44) and not for the first time period which is day 30 .

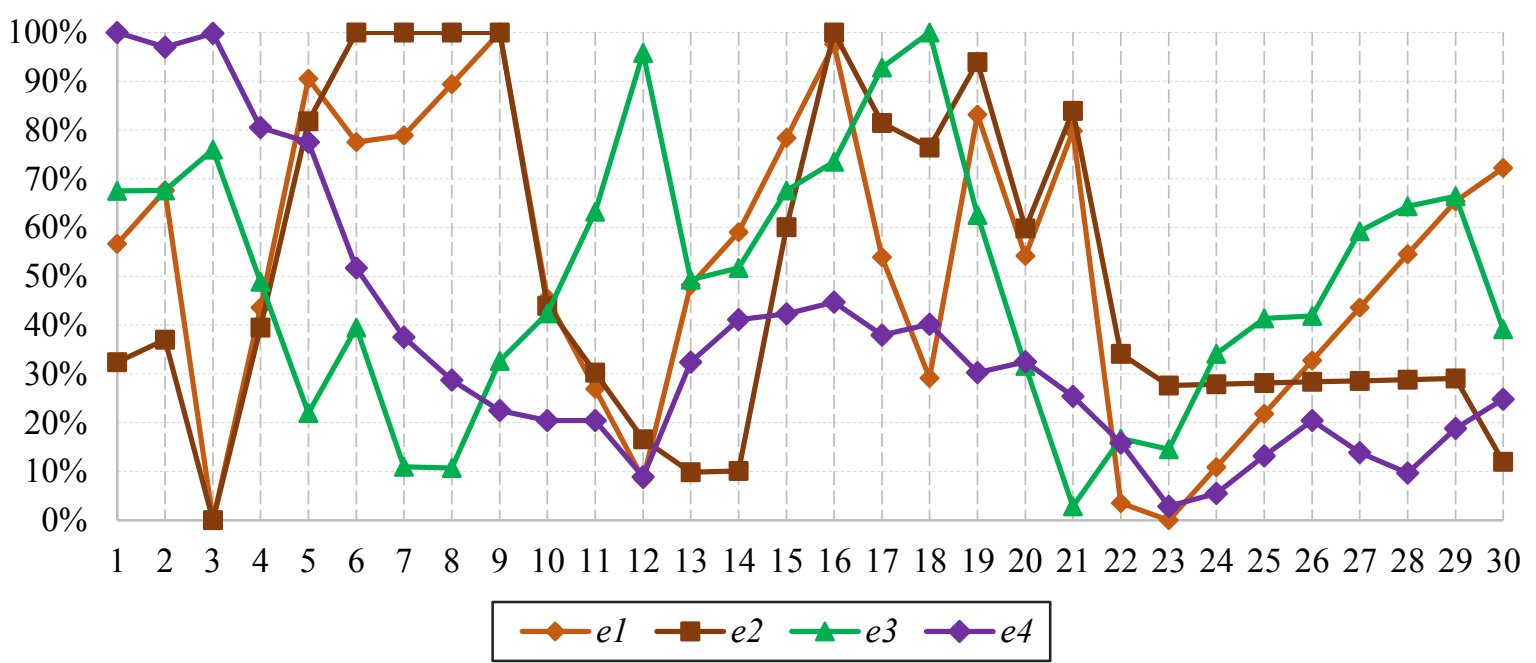

Figure 31. Case Study 3 - Rolling Horizon Integrated Approach: Normalized inventory profiles for utility and product resources.

Figure 31 displays the normalized inventory profiles for utility and product resources, having as reference the associated maximum inventory levels. The high inventory levels for utility and product resources at the first period is due to the high initial inventory levels. There are reduced inventory levels for utility resources from day 10 to 12 and from day 16 to 18 due to the offline cleaning of some utility units that takes place in these days (see Figure 26). The inventory levels for product resources are reduced on day 4 to 7 and day 19 to 21 because of offline cleanings for production units. Recall that all inventory tanks are subject to terminal constraints that force the inventory levels in the last time period of each iteration to be $25 \%$ of the maximum capacity of the corresponding inventory tank. According to Figure 31, the inventory level for utility resource $e 2$ in day 30 is below $25 \%$ but this is not a violation of the terminal constraints. The solution of day 30 (including the inventory level values) has been derived from iteration 30 by solving a planning problem from time period 30 to time period 44, satisfying the terminal constraints for time period 44 .

\subsubsection{Results of Case Study 3 - Sequential Approach}


Figure 32 displays the final Gantt chart and total utilization profile of cleaning resources for the sequential rolling horizon approach. In comparison with the integrated approach, a higher number of offline and online cleaning tasks for utility units is observed. Utility units i4 and $i 5$ operate in a larger number of time periods than before. Also, production unit $i 7$ is utilized in this case, while in the solution from the integrated rolling horizon approach was inactive for the whole planning horizon (see Figure 27). Here, production unit $i 7$ operates at the first half of the planning horizon and production unit is operates mostly at the second half of the planning horizon. This solution also reports a highly increased number of production changeovers in the production units, which in practice can make more complicate the implementation of this plan.

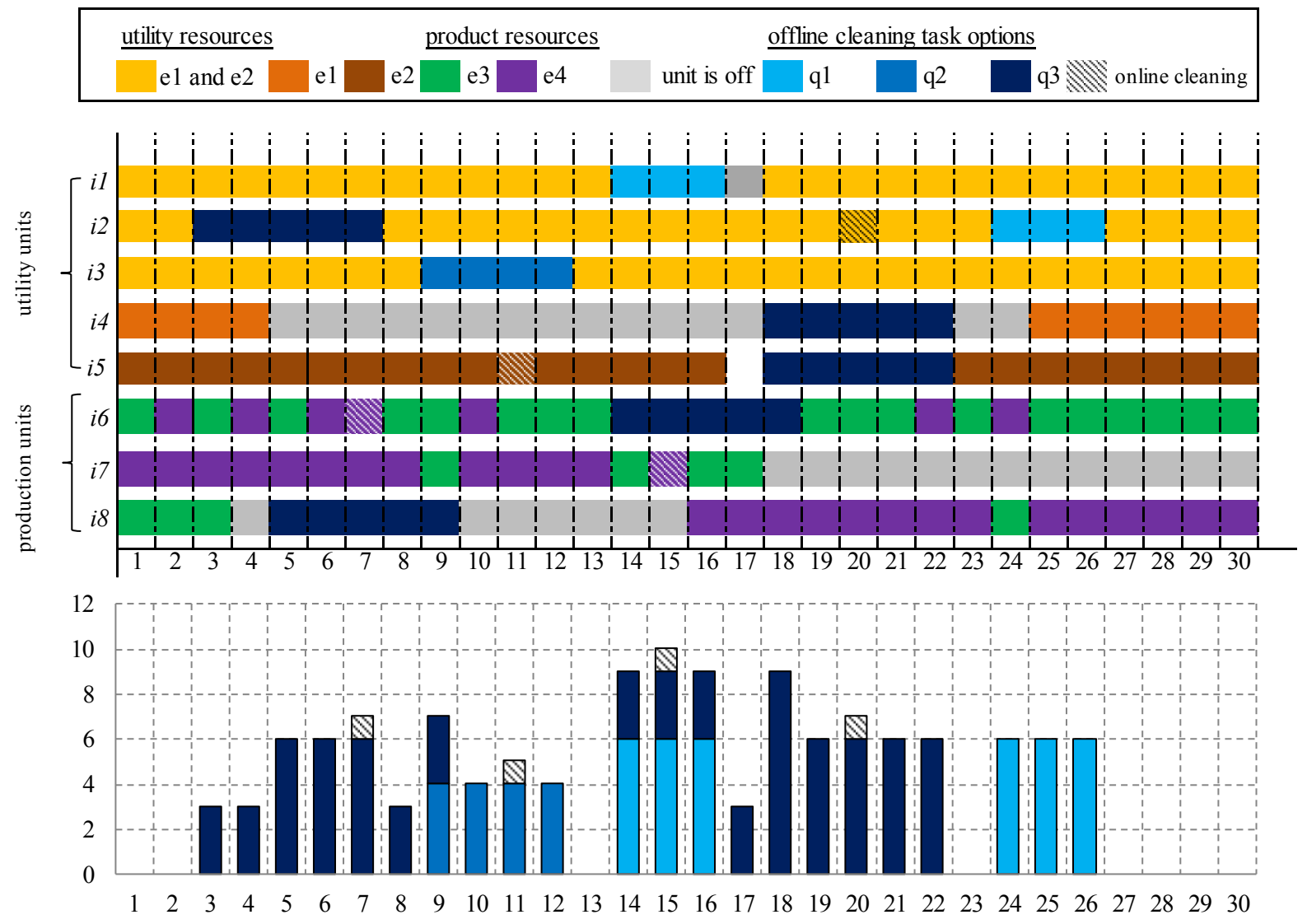


Figure 32. Case Study 3 - Rolling Horizon Sequential Approach: Operational and cleaning plan for production and utility systems and total utilization profile of cleaning resources.

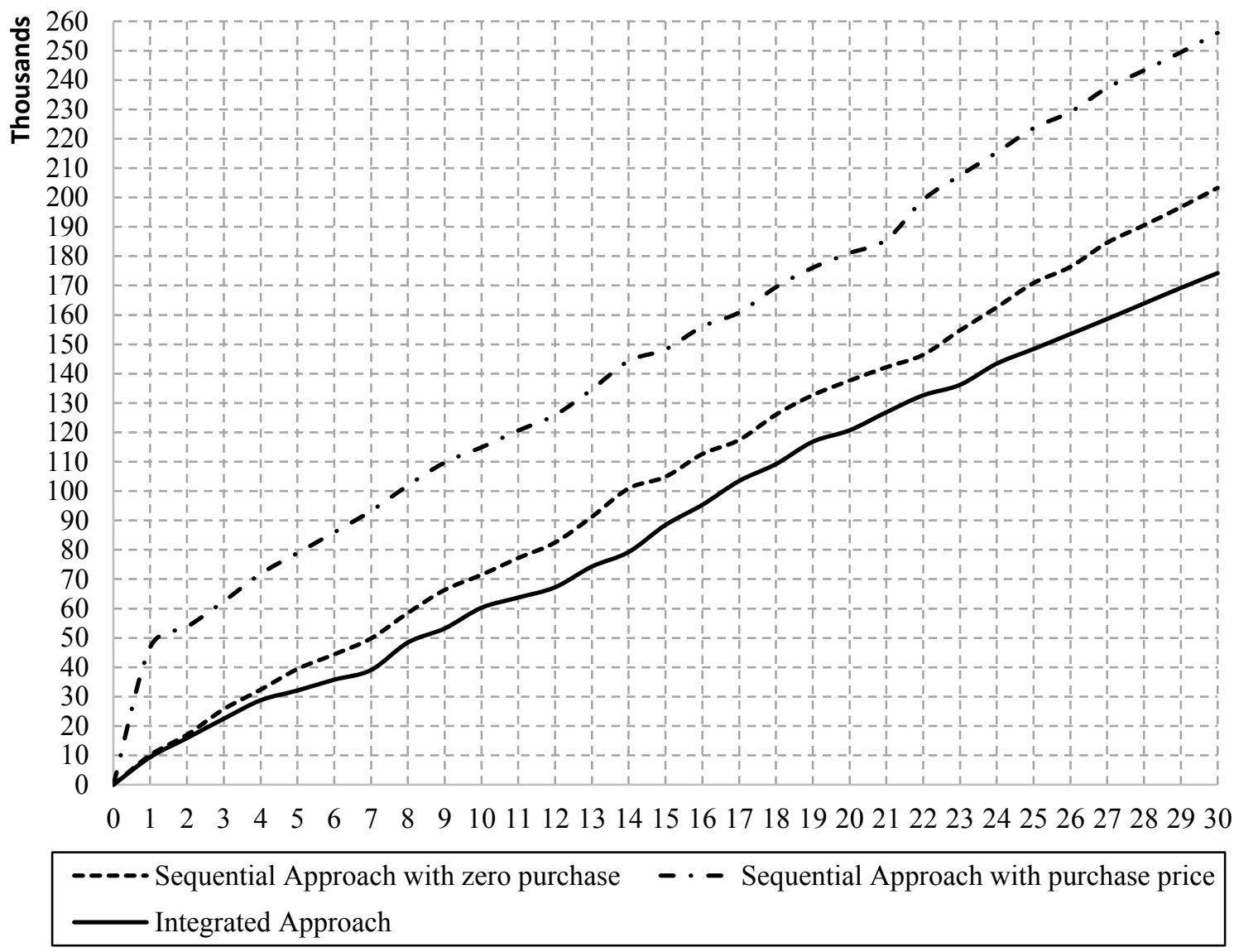

Figure 33. Case Study 3: Aggregated total cost for integrated and sequential rolling horizon approaches.

Figure 33 displays the aggregated total cost for the integrated and the sequential rolling horizon approach. The total cost of the integrated approach is $14 \%$ lower than that of the sequential approach if a zero purchase price is considered, and 32\% lower than that of the sequential approach if a purchase price equal to 200 is considered. The results clearly show that the integrated approach can find solutions that are better than those of the sequential approach, even if a zero purchase price is considered. In practice, penalty or real costs for acquiring utilities from external sources can be very high, since either represent an undesired managerial policy (i.e., dependency on external sources) or high-cost utilities. In this example, the solution 
following the sequential approach reports a total of 263.8 units of utility resource $e 2$ that need to be purchased from external sources, as shown in Table 10.

Table 10. Case study 3: Sequential rolling horizon approach. Utilities purchases Utility Resource Amount per time period (in metric units) Total (in metric units)

\begin{tabular}{lllllll}
\hline & day 1 & day 4 & day 6 & day 7 & day 22 & \\
$e 2$ & 183.6 & 13.9 & 10.4 & 9.2 & 46.8 & 263.8 \\
\hline
\end{tabular}

Extra power consumption cost

Cleaning cost for units

Operating cost for production units

Operating cost for utility units

Startup and shutdown

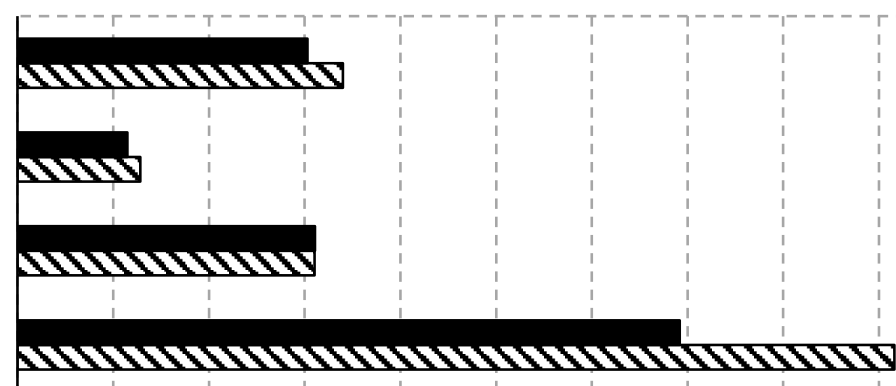

NDNONONO

$\begin{array}{lllllllllll}0 \% & 5 \% & 10 \% & 15 \% & 20 \% & 25 \% & 30 \% & 35 \% & 40 \% & 45 \% & 50 \%\end{array}$

- Integrated Approach $\mathbf{\Delta}$ Sequential Approach

Figure 34. Case Study 3: Cost comparison of integrated and sequential rolling horizon approaches.

Figure 34 shows the cost comparison of the solutions derived by following the integrated and the sequential rolling horizon approach. Note that this figure does not include the purchase cost for resources. As in the previous case study, the highest difference is observed in the operating cost for utility units by about $11 \%$. Extra energy consumption cost difference is at $2 \%$. The cleaning cost and startup and shutdown cost report both a difference of around $0.6 \%$. The operating cost for production units is almost the same for both approaches. 


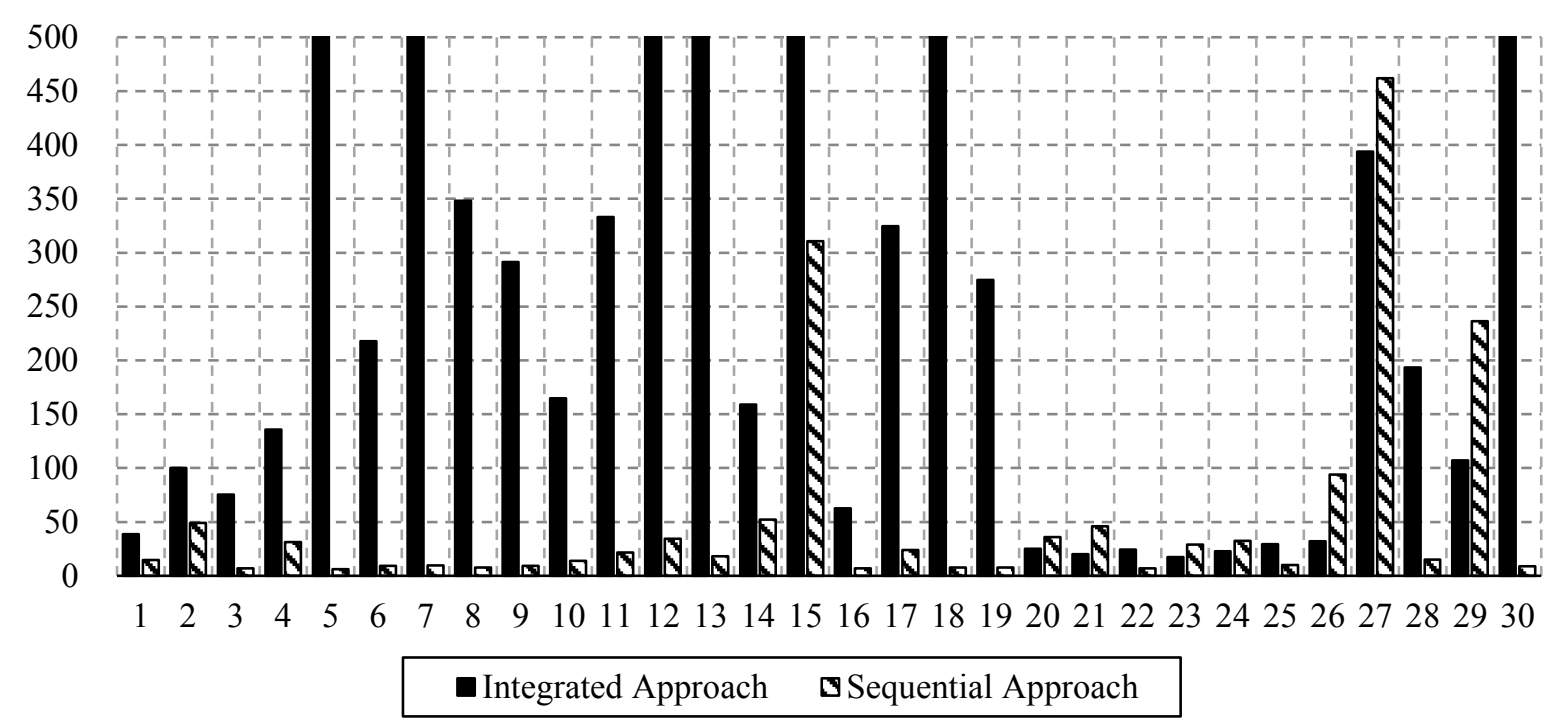

Figure 35. Case Study 3: CPUs values per iteration for integrated and sequential rolling horizon approaches.

Figure 35 shows the CPUs values of each iteration for both approaches. In most of the iterations, the integrated approach shows much higher CPUs values than the sequential approach. The average computational times for the sequential and the integrated approach are 53.9 and 389 CPUs, respectively. It should be clear that the integrated planning problem results in a more complex optimization problem than the sequential planning problem, and therefore higher computational times would be observed for the resolution of the same planning problem. In Figure 35, one can observe that in some iterations, such as iteration 27 and 29, the computational time of the sequential approach is higher than that of the integrated approach. This is due to the fact that the two approaches may not solve exactly the same problem at each iteration (apart from the first iteration), since the planning problem under optimization at each operation depends strongly on the initial state of the system, which in the rolling horizon framework is an optimization output of the previous iteration (apart from the first iteration). Considering the complexity of the integrated planning problems solved in each iteration, the integrated approach reported a very good computational performance. 


\section{Conclusions}

In this study, a rolling horizon optimization framework has been developed for the integrated condition-based planning of utility and production system under uncertainty. Performance degradation and recovery has been considered for both systems. A number of representative case studies showed that the proposed integrated approach can provide significantly better solutions (compared to solutions obtained by sequential approaches) in terms of total costs, and especially in cost terms related to utility units operation, extra energy consumption, cleaning and startup/shutdown operations. With respect to our previous work, improved unit performance degradation and recovery models that depend on both the cumulative time of operation and the unit operating levels deviation of units have been developed. This is a major step for addressing industrial scenarios. In the case studies solved, we observed that the total cost of the solution of the integrated approach is lower than that of the solution of sequential approach within a range of $5 \%$ to $32 \%$. This significant reduction in total costs is a direct result of the enhanced energy efficiency of the overall system through the optimized use and consumption of energy (i.e., major parts of the objective function). It has been also demonstrated that unnecessary purchases of resources can be avoided by the proposed integrated approach through the more efficient operation of utility units and the improved utilization handling of energy and material resources. Overall, the proposed approach can result in a cleaner production since energy generation and consumption along with cleaning operations plans (source of waste sources) are optimized. In the longer term this could result in a sustainable production practices. Ongoing research activities focus on the modeling of more complex production processes along with the development of decomposition methods for the effective solution of such highly complicated planning problems. 


\section{Acknowledgments}

The authors would like to express their gratitude to the Ministry of Higher Education Malaysia for providing financial support under the Scheme of Academic Training Reward throughout the realization of this research work.

\section{NOMENCLATURE}

Indices / Sets

$\begin{array}{ll}e \in E & \text { resources (products and utilities) } \\ i \in I & \text { units (production and utility) } \\ t \in Q & \text { offline cleaning task options } \\ z \in Z & \text { time periods } \\ & \text { inventory tanks for resources }\end{array}$

\section{Superscripts}

$\begin{array}{ll}\text { es } & \text { earliest } \\ \text { ls } & \text { latest } \\ \text { max } & \text { maximum } \\ \text { min } & \text { minimum } \\ \text { off } & \text { offline } \\ \text { on } & \text { online } \\ \text { s } & \text { startup } \\ \text { f } & \text { shutdown } \\ \text { fix } & \text { fixed } \\ \text { var } & \text { variable } \\ \text { PR } & \text { production system } \\ \text { UT } & \text { utility system }\end{array}$




$$
\begin{array}{cc}
+ & \text { inlet } \\
- & \text { outlet }
\end{array}
$$

\section{Subsets}

$E_{i} \quad$ resources that can be produced in unit $i$

$E^{P R} \quad$ product resources

$E^{U T} \quad$ utility resources

$I_{e} \quad$ units that can produced resource $e$

$I^{S F} \quad$ units that are subject to startup and shutdown costs

$I^{S-\min } \quad$ units that are subject to minimum runtimes

$I^{F-\min } \quad$ units that are subject to minimum shutdown times

$I_{e}^{P R} \quad$ production units that require utility resource $e$ to operate

$Q_{i} \quad$ alternative offline cleaning task options for unit $i$

$Z_{e} \quad$ inventory tanks that can store resource $e$

$C B_{i} \quad$ units $i$ that are subject to condition-based cleaning tasks

$D M_{i} \quad$ units $i$ that are under in-progress offline cleaning at the beginning of the planning horizon (information carried over from previous planning horizon)

$F M_{i} \quad$ units $i$ that are subject to flexible time-window offline cleaning

$M R_{i} \quad$ units $i$ that are subject to maximum runtime constraints

$P R_{i} \quad$ production units

$U T_{i} \quad$ utility units

\section{Parameters}

$\alpha_{\left(i, e, e^{\prime}\right)}$

coefficient for production unit $i$ that provides the variable needs for utility $e$ for the production of a unit of product $e^{\prime}$ 
coefficient for production unit $i$ that provides the fixed needs for utility resources $e$ for the production of resources $e^{\prime}$ coefficient of losses in inventory tank $z$ minimum time between two consecutive online cleanings in unit $i$

$\delta_{i}$ performance degradation rate for unit $i$ due to its cumulative time of operation

$\delta_{i}^{q} \quad$ performance coefficient related to operating level for unit $i$ due to its cumulative deviation from its reference operating level

$\varepsilon_{(e, z, t)} \quad$ bounds on the total inlet/outlet flow of resource $e$ to/from inventory tank $z$ in time period $t$

$\zeta_{(e, t)} \quad$ demand for product resource $e \in E^{P R}$ in time period $t$

$\eta_{t}^{\max } \quad$ limited amount of available resources for cleaning operations in time period $t$

$\vartheta_{(i, q)}^{\text {off }} \quad$ resource requirements for offline cleaning task option $q$ of unit $i$

$\vartheta_{i}^{o n} \quad$ resource requirements for online cleaning of unit $i$

$\kappa_{(i, t)} \quad$ bounds on the operating level for utility unit $i \in U T_{i}$ in time period $t$

$\bar{\kappa}_{(i, e, t)} \quad$ bounds on the production level of product resource $e \in E^{P R}$ for production unit $i \in P R_{i}$ in time period $t$

$\lambda_{(e, z)}^{B} \quad$ percentage coefficient that determines the minimum level for each resource inventory tank at the end of the prediction horizon (terminal value)

$\lambda_{i}^{U} \quad$ percentage coefficient that determines the maximum extra energy consumption level for operating unit $i$ at the end of the prediction horizon (terminal value) $\mu_{(i, t)}, \bar{\mu}_{(i, t)} \quad$ sufficient big numbers duration of offline cleaning task option $q$ that could take place in unit $i$ 


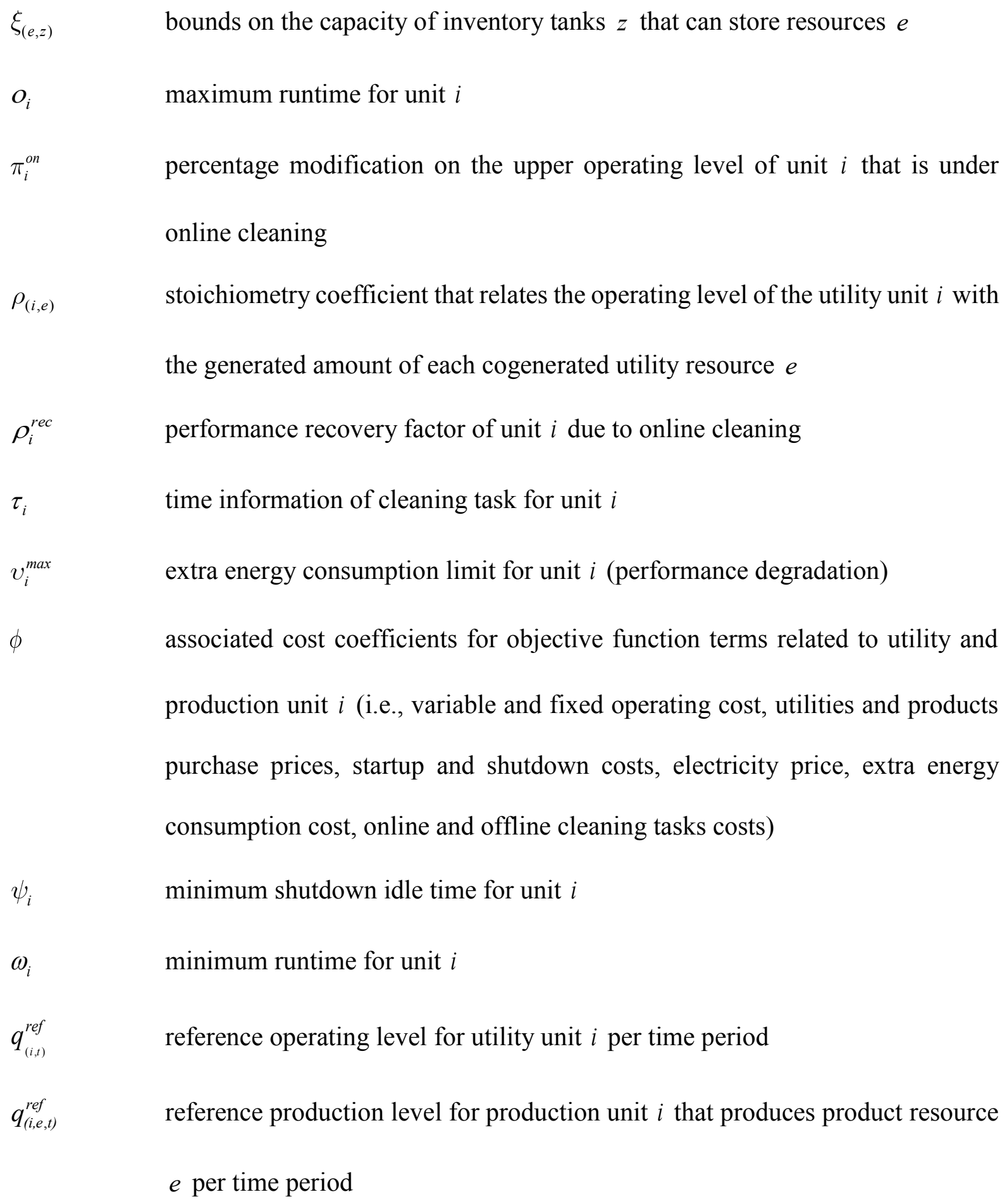

\section{Parameters (initial state of the overall system)}

$\tilde{\beta}_{(e, z)} \quad$ initial inventory level of resource $e$ in inventory tank $z$

$\tilde{\gamma}_{i}^{o n} \quad$ initial state of utility unit $i \in C B_{i}^{o n}$ with respect to its last online cleaning 
$\tilde{\eta}_{(i, t)} \quad$ time periods $t$ for utility unit $i \in D M_{i}$ that there is a known cleaning resource requirement (in-progress offline cleaning task from previous planning horizon)

$\tilde{\rho}_{i} \quad$ initial cumulative time of operation for unit $i$

$\tilde{\rho}_{i}^{q} \quad$ initial cumulative deviation from the reference operating level for unit $i$

$\tilde{\chi}_{i} \quad$ operating status of unit $i$ just before the beginning of the current planning horizon

$\tilde{\psi}_{i} \quad$ total number of time periods at the beginning of the current planning horizon that unit $i$ has been continuously not operating since its last shutdown

$\widetilde{\omega}_{i} \quad$ total number of time periods at the beginning of the current planning horizon that unit $i$ has been continuously operating since its last startup

\section{Continuous variables (non-negative)}

$B_{(e, z, t)} \quad$ inventory level for resource $e$ in inventory tank $z$ at time period $t$

$B_{(e, z, t)}^{-} \quad$ total outlet flow of resource $e$ from inventory tank $z$ at time period $t$

$B_{(e, z, t)}^{+} \quad$ total inlet flow of resource $e$ to inventory tank $z$ at time period $t$

$B_{(e, z, i, t)}^{U T,-} \quad$ flow of utility $e$ from inventory tank $z$ to production unit $i$ at time period $t$

$D_{(i, t)} \quad$ cumulative operating level deviation for unit $i$ in time period $t$

$N S_{(e, i, t)}^{U T} \quad$ purchases of utility resource $e$ to be utilized in production unit $i \in I_{e}^{P R}$ in time period $t$

$N S_{(e, t)}^{F P} \quad$ purchases of product resource $e$ in time period $t$ (or lost sales)

$\bar{Q}_{(i, t)} \quad$ operating level of utility unit $i$ in time period $t$

$Q_{(i, e, t)} \quad$ production level of resource $e$ from unit $i$ in time period $t$

$R_{(i, t)} \quad$ cumulative time of operation for unit $i$ in time period $t$ 
$U_{(i, t)} \quad$ extra energy consumption (from fully clean condition) of unit $i$ due to its performance degradation

\section{Binary variables}

$X_{(i, t)} \quad=1$, if a unit $i$ is operating during time period $t$

$S_{(i, t)} \quad=1$, if a unit $i$ starts up at the beginning of time period $t$

$F_{(i, t)} \quad=1$, if a unit $i$ shuts down at the beginning of time period $t$

$V_{(i, t)} \quad=1$, if an online cleaning task for unit $i \in C B_{i}^{o n}$ occurs in time period $t$

$V_{(i, e, t)}^{P R} \quad=1$, if an online cleaning task for production unit $i \in\left(P R_{i} \cap C B_{i}^{o n}\right)$ that produces product resource $e \in E^{P R}$ takes place in time period $t$

$W_{(i, t)} \quad=1$, if an offline cleaning task for unit $i \in\left(C B_{i}^{\text {off }} \cup F M_{i}\right)$ starts at the beginning of time period $t$

$H_{(i, q, t)} \quad=1$, if the offline cleaning task option $q \in Q_{i}$ for unit $i \in\left(C B_{i}^{\text {off }} \cup F M_{i}\right)$ starts at the beginning of time period $t$

$Y_{(i, e, t)}=1$, if production unit $i \in P R_{i}$ produces product resource $e$ in time period $t$

\section{References}

Abdul Aziz, E., Wan Alwi, S.R., Lim, J.S., Abdul Manan, Z., Klemeš, J.J., 2017. An integrated pinch analysis framework for low CO2 emissions industrial site planning. J. Clean. Prod. $146,125-138$.

Agha, M.H., Thery, R., Hetreux, G., Hait, A., Le Lann, J.M., 2010. Integrated production and utility system approach for optimizing industrial unit operations. Energy 35, 611-627.

Ardjmand, E., Weckman, G.R., Young, W.A., Sanei Bajgiran, O., Aminipour, B., 2016. A robust optimisation model for production planning and pricing under demand uncertainty. Int. J. Prod. Res. 54, 3885-3905. 
Castro, P.M., Grossmann, I.E., Veldhuizen, P., Esplin, D., 2014. Optimal maintenance scheduling of a gas engine power plant using generalized disjunctive programming. AIChE J. 60, 2083-2097.

Chaturvedi, N.D., Manan, Z.A., Wan Alwi, S.R., Bandyopadhyay, S., 2016. Effect of multiple water resources in a flexible-schedule batch water network. J. Clean. Prod. 125.

Cheung, K.Y., Hui, C.W., 2004. Total-site scheduling for better energy utilization. J. Clean. Prod. 12, 171-184.

Goel, H.D., Grievink, J., Weijnen, M.P.C., 2003. Integrated optimal reliable design, production, and maintenance planning for multipurpose process plants. Comput. Chem. Eng. 27, 1543-1555.

Huang, R.H., Yu, S.C., 2016. Two-stage multiprocessor flow shop scheduling with deteriorating maintenance in cleaner production. J. Clean. Prod. 135, 276-283.

Jin, S.W., Li, Y.P., Huang, G.H., Zhang, K., 2015. Inexact mixed-integer programming with interval-valued membership function for sustainable power-generation capacity planning. J. Clean. Prod. 122, 52-66.

Kopanos, G.M., Pistikopoulos, E.N., 2014. Reactive scheduling by a multiparametric programming rolling horizon framework: A case of a network of combined heat and power units. Ind. Eng. Chem. Res. 53, 4366-4386.

Kopanos, G.M., Xenos, D.P., Cicciotti, M., Pistikopoulos, E.N., Thornhill, N.F., 2015. Optimization of a network of compressors in parallel: Operational and maintenance planning - The air separation plant case. Appl. Energy 146, 453-470.

Lavaja, J.H., Bagajewicz, M.J., 2004. On a new MILP model for the planning of heatexchanger network cleaning文. Ind. Eng. Chem. Res. 43, 3924-3938.

Li, Y.G., Nilkitsaranont, P., 2009. Gas turbine performance prognostic for condition-based maintenance. Appl. Energy 86, 2152-2161. 
Modarres, M., Izadpanahi, E., 2016. Aggregate production planning by focusing on energy saving: A robust optimization approach. J. Clean. Prod. 133, 1074-1085.

Nguyen, D., Bagajewicz, M., 2010. Optimization of preventive maintenance in chemical process plants. Ind. Eng. Chem. Res. 49, 4329-4339.

Sanaye, S., Niroomand, B., 2007. Simulation of heat exchanger network (HEN) and planning the optimum cleaning schedule. Energy Convers. Manag. 48, 1450-1461.

Shrouf, F., Ordieres-Meré, J., García-Sánchez, A., Ortega-Mier, M., 2014. Optimizing the production scheduling of a single machine to minimize total energy consumption costs. J. Clean. Prod. 67, 197-207.

Tayyab, M., Sarkar, B., 2016. Optimal batch quantity in a cleaner multi-stage lean production system with random defective rate. J. Clean. Prod. 139, 922-934.

Xenos, D.P., Kopanos, G.M., Cicciotti, M., Thornhill, N.F., 2016. Operational optimization of networks of compressors considering condition-based maintenance. Comput. Chem. Eng. $84,117-131$.

Zhang, B.J., Hua, B., 2007. Effective MILP model for oil refinery-wide production planning and better energy utilization. J. Clean. Prod. 15, 439-448.

Zhen, J.L., Huang, G.H., Li, W., Wu, C.B., Wang, S., 2016. Electric power system planning with renewable energy accommodation for supporting the sustainable development of Tangshan City, China. J. Clean. Prod. 139, 1308-1325.

Zhou, L., Xu, K., Cheng, X., Xu, Y., Jia, Q., 2017. Study on optimizing production scheduling for water-saving in textile dyeing industry. J. Clean. Prod. 141, 721-727.

Zulkafli, N.I., Kopanos, G.M., 2016. Planning of production and utility systems under unit performance degradation and alternative resource-constrained cleaning policies. Appl. Energy 183, 577-602. 